\title{
Reflexão de funções cardinais e da metrizabilidade
}

\author{
Rodrigo Roque Dias
}

DISSERTAÇÃO APRESENTADA

$\mathrm{AO}$

Instituto de MATEMÁticA e EstatísticA

DA

Universidade De SÃo Paulo

PARA

OBTENÇÃO DO TÍTULO

$\mathrm{DE}$

Mestre em CiÊnCIAS

\section{Área de concentração: Matemática \\ Orientadora: Profa. Dra. Lúcia Renato Junqueira}

Durante o desenvolvimento deste trabalho, o autor recebeu auxílio financeiro do CNPq Conselho Nacional de Desenvolvimento Científico e Tecnológico

São Paulo, agosto de 2008 



\title{
Reflexão de funções cardinais e da metrizabilidade
}

\author{
Este exemplar corresponde à redação \\ final da dissertação devidamente corrigida \\ e defendida por Rodrigo Roque Dias \\ e aprovada pela Comissão Julgadora.
}

Banca Examinadora:

- Profa. Dra Lúcia Renato Junqueira (orientadora) - IME-USP.

- Prof. Dr. Ofelia Teresa Alas - IME-USP.

- Prof. Dr. Marcelo Dias Passos - UNIFESP. 

A Marly, Francisco e Rita 



\section{Agradecimentos}

Vou evitar ao máximo citar nomes, para não correr o risco de me esquecer de alguém.

Aos meus pais, Marly e Francisco, e à minha irmã, Rita de Cássia, simplesmente por tudo.

Aos professores e colegas que me acompanharam em minha formação acadêmica, a quem eu devo a maior parte do que sei hoje. Há um pouco de vocês em cada linha deste trabalho.

Aos amigos que estiveram ao meu lado o tempo todo. Vocês fizeram uma enorme diferença.

Ao $\mathrm{CNPq}$, pelo apoio financeiro nesses dois anos.

A Marcelo Dias Passos e Samuel Gomes da Silva, pelas críticas e sugestões ao trabalho final.

A Ricardo Bianconi, por todo o apoio — desde a iniciação científica até hoje.

A Ofelia Teresa Alas, pela acolhida, pela inestimável ajuda e, talvez o mais importante, pelo exemplo.

E, é claro, àquela que me guiou por estas páginas e tornou tudo isto possível. Prefiro não tentar listar os motivos, para não correr o risco de me esquecer de algum. Obrigado, Lúcia. 

Quanto mais estudo, mais descubro a vastidão de minha ignorância. Jorge Furtado 



\section{Resumo}

O conceito de reflexão em topologia expressa o fato de que um espaço satisfaz uma dada propriedade sempre que esta é satisfeita por seus subespaços "menores". Neste trabalho, estuda-se a reflexão de propriedades envolvendo a maioria das principais funções cardinais e metrizabilidade, bem como outras propriedades relacionadas. São discutidos problemas em aberto - como o problema de Hamburger —, incluindo respostas parciais e exemplos de consistência. Várias dentre as demonstrações apresentadas utilizam técnicas de submodelos elementares, que constituem hoje uma importante ferramenta no estudo de topologia geral.

Palavras-chave: reflexão, funções cardinais, metrizabilidade, espaços coletivamente de Hausdorff, bases pontualmente enumeráveis 


\section{Abstract}

The concept of reflection in topology expresses the fact that a space satisfies a given property provided that its "small" subspaces do. This work presents a study on reflection of properties concerning most of the main cardinal functions and metrizability, as well as other related properties. Open problems - such as Hamburger's question - are also discussed, including partial answers and consistent examples. Several of the proofs presented here make use of elementary submodels, nowadays an important tool in the study of general topology.

Keywords: reflection, cardinal functions, metrizability, collectionwise Hausdorff spaces, pointcountable bases 


\section{Sumário}

1 Preliminares $\quad 5$

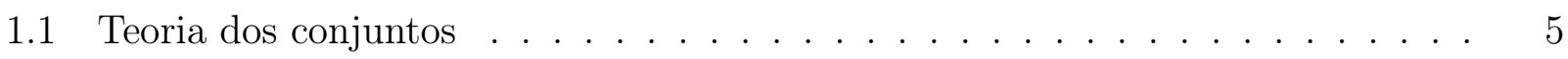

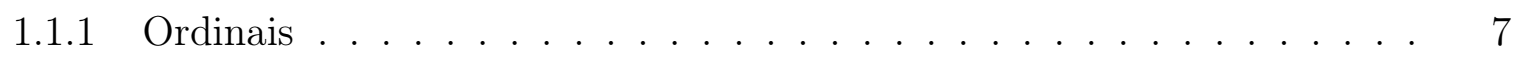

$1.1 .2 \quad \mathrm{O}$ princípio $\square_{\kappa} \ldots \ldots \ldots \ldots$

1.1.3 Submodelos elementares ........................ 9

1.2 Topologia geral . . . . . . . . . . . . . . . . . . . 22

1.2.1 Definições e resultados básicos . . . . . . . . . . . . . . . . . . 22

1.2.2 Propriedades de separação . . . . . . . . . . . . . . . . 23

1.2.3 A topologia da ordem . . . . . . . . . . . . . . 26

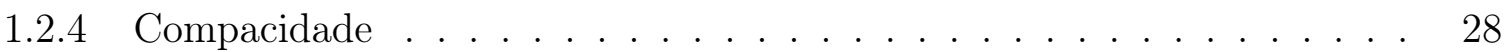

1.2.5 Metrizabilidade ...................... 31

1.2.6 Um teorema de Miščenko . . . . . . . . . . . . . . . . . . . 32

1.2.7 Funções cardinais . . . . . . . . . . . . . . . . . . . . . . . . . 34

2 Reflexão de funções cardinais $\quad 41$

2.1 Teoria geral . . . . . . . . . . . . . . . . . . . . 41 
2.2 Funções cardinais globais . . . . . . . . . . . . . . . . . . . . . . . 45

2.2.1 Reflexão de $e, s$ e $c \ldots \ldots \ldots \ldots$. . . . . . . . . . . . . . . . . .

2.2.2 Reflexão de $d$ e $h d \ldots \ldots \ldots \ldots$

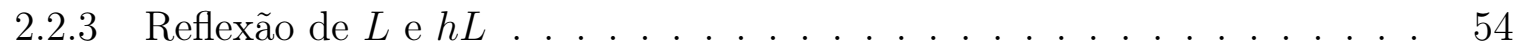

2.2 .4 Reflexão de $w \ldots \ldots \ldots \ldots$. . . . . . . . . . . . . 59

2.3 Funções cardinais locais . . . . . . . . . . . . . . . . . . . . . . . . 62

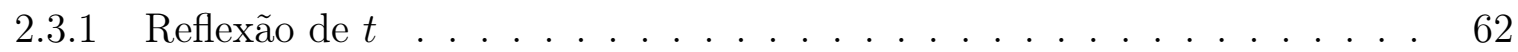

2.3.2 Reflexão de $\chi \ldots \ldots \ldots \ldots$. . . . . . . . . . . . . . . . . .

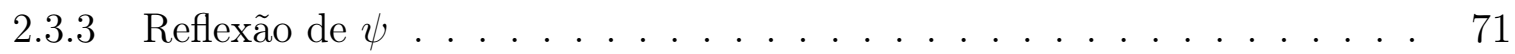

2.4 Problemas em aberto . . . . . . . . . . . . . . . . . 73

3 Reflexão da metrizabilidade $\quad 75$

3.1 O problema de Hamburger . . . . . . . . . . . . . . . . . . . . . 75

3.2 Espaços $\omega_{1}$-coletivamente de Hausdorff . . . . . . . . . . . . . . . . . . . . 84

3.3 Reflexão de bases pontualmente enumeráveis . . . . . . . . . . . . . . . 88

$\begin{array}{ll}\text { A O lema da canonização } & 97\end{array}$

$\begin{array}{ll}\text { B Sobre um exemplo de A. Dow } & 109\end{array}$

B.1 Propriedades do espaço $(X, \tau) \ldots \ldots \ldots \ldots \ldots$

$\begin{array}{lr}\text { Índice de símbolos } & 121\end{array}$

$\begin{array}{lr}\text { Índice remissivo } & 122\end{array}$ 
Referências bibliográficas 
xviii 


\section{Introdução}

Certas propriedades topológicas, ao serem verificadas por subespaços "menores" de um espaço topológico, são necessariamente verificadas pelo mesmo. Para exemplificar tal fato, citamos os seguintes resultados - cabe observar que, em alguns casos, é necessário assumir alguma hipótese sobre o espaço considerado -:

Teorema (Tkachuk, [31]). Seja X um espaço de Hausdorff tal que $\bar{D}$ é compacto para todo $D \subseteq X$ discreto. Então $X$ é compacto.

Teorema (Hajnal e Juhász, [16]). Sejam $\kappa$ um cardinal não-enumerável e $X$ um espaço topológico tais que todo $Y \subseteq X$ com $|Y| \leq \kappa$ admite uma base de abertos de cardinalidade menor que $\kappa$. Então $X$ admite uma base de abertos de cardinalidade menor que $\kappa$.

Teorema (Dow, [5]). Seja X um espaço de Hausdorff enumeravelmente compacto tal que todo $Y \subseteq X$ com $|Y| \leq \omega_{1}$ é metrizável. Então $X$ é metrizável.

Teoremas desse tipo recebem o nome de teoremas de reflexão e constituem um interessante campo de pesquisa em topologia geral - para um panorama mais amplo sobre este tópico e outros problemas com os quais ele se relaciona, vide, e.g., [30].

O objeto de estudo desta dissertação são resultados de reflexão como os dois últimos citados acima ${ }^{1}$, em que, por "menor", se entende "que tem cardinalidade menor ou igual a $\kappa$ ", sendo $\kappa$ um cardinal infinito. Mais especificamente, são tratados resultados de reflexão acerca

\footnotetext{
${ }^{1}$ Os quais se encontram demonstrados no texto, respectivamente, como o teorema 2.2.20 e o corolário 3.1.9.
} 
de funções cardinais e metrizabilidade - e propriedades relacionadas —, como detalharemos a seguir.

O primeiro capítulo tem por objetivo listar os principais conceitos e resultados que serão utilizados ao longo do texto, bem como fixar certas notações e terminologias empregadas no mesmo. Destacamos nesse capítulo a seção dedicada ao tópico de submodelos elementares, na qual se desenvolvem as principais ferramentas relacionadas a tal conceito visando sua aplicação na obtenção de resultados em topologia geral, como será feito nos capítulos posteriores.

O segundo capítulo, que tem [18] como referência principal, é dedicado ao estudo de teoremas de reflexão envolvendo certas funções cardinais. O interesse se concentra, mais precisamente, em resultados do tipo

"Se $X \in \mathcal{S}$ é tal que $\varphi(Y)<\kappa$ para todo $Y \subseteq X \operatorname{com}|Y| \leq \kappa$, então $\varphi(X)<\kappa$ ",

em que $\mathcal{S}$ é uma classe de espaços topológicos - e.g., $\mathcal{S}=\{X \mid X$ é um espaço de Hausdorff $\}$ - $\varphi$ é uma função cardinal e $\kappa$ é um cardinal infinito; como veremos no texto, resultados dessa forma se relacionam ainda a outras propriedades que $\varphi$ apresenta em $\mathcal{S}$.

No terceiro - e último - capítulo, a atenção volta-se a resultados de reflexão sobre metrizabilidade. A motivação principal é o chamado problema de Hamburger, proposto por P. Hamburger na década de 1970 — vide [15] — ao perguntar se é necessariamente metrizável um espaço normal $X$ de caráter enumerável tal que todo $Y \subseteq X$ com $|Y| \leq \omega_{1}$ é metrizável; tendo permanecido sem resposta desde então, é considerado hoje um dos problemas clássicos da topologia geral — vide [29] e [30]. Neste capítulo da dissertação, são apresentados resultados positivos assumindo-se hipóteses topológicas adicionais - como, por exemplo, o último dos três teoremas enunciados no primeiro parágrafo desta introdução - , bem como contra-exemplos consistentes. Parte dos resultados e contra-exemplos relacionam-se a outros problemas a saber, a reflexão de bases pontualmente enumeráveis e a existência de espaços de caráter enumerável que são $\omega_{1}$-coletivamente de Hausdorff mas não são coletivamente de Hausdorff —, tratados com maior detalhe, respectivamente, nas seções 3.3 e 3.2. 
Por fim, no apêndice A é feita a demonstração do lema da canonização, um resultado de combinatória infinita utilizado na demonstração do teorema 2.2.16, e o apêndice B é um estudo parcial sobre um exemplo proposto por A. Dow em [5] relacionado às propriedades de reflexão da função cardinal $\chi$. 


\section{Capítulo 1}

\section{Preliminares}

Este capítulo, naturalmente, não tem o intuito de desenvolver toda a teoria necessária para a compreensão desta dissertação; seu propósito é, antes, fixar as nomenclaturas e notações ${ }^{1}$ empregadas no texto, bem como listar resultados que serão utilizados ao longo do mesmo. Uma exceção é feita na seção 1.1.3, que consiste numa breve introdução à teoria de submodelos elementares.

Definições, notações e resultados utilizados neste trabalho e que não constam deste capítulo podem ser encontrados em [19], [25] ou [8].

\section{$1.1 \quad$ Teoria dos conjuntos}

Como de praxe, as abreviaturas $\mathrm{ZFC}, \mathrm{CH}, \mathrm{GCH}$, MA e $V=L$ denotam, respectivamente, a axiomática de Zermelo-Fraenkel acrescida do axioma da escolha, a hipótese do contínuo, a hipótese do contínuo generalizada, o axioma de Martin e o axioma da construtibilidade.

\footnotetext{
${ }^{1}$ Em especial com relação a conceitos em que não há uniformidade nas mesmas, ou a outros que eventualmente não são abordados num primeiro contato com a teoria dos conjuntos e a topologia geral.
} 
Assume-se ZFC em todo o decorrer desta dissertação ${ }^{2}$. Em certos pontos, faremos referência a classes; em particular, denotaremos por $\mathcal{T}$, Ord e Card, respectivamente, a classe de todos os espaços topológicos, a classe de todos os ordinais e a classe de todos os cardinais.

Classes não serão tratadas formalmente neste trabalho - como o fazem certas teorias de conjuntos diferentes de ZFC-; nosso interesse ao utilizar tal conceito será unicamente simplificar a notação e a linguagem empregadas, de modo a facilitar a compreensão dos tópicos. ${ }^{3}$ Por exemplo, por $X \in \mathcal{T}$ entenda-se apenas " $X$ é um espaço topológico". Utilizaremos para classes as notações $\mathcal{X} \subseteq \mathcal{Y}$ e $x \in \mathcal{X} \cap \mathcal{Y}$ significando, respectivamente, " $\forall x(x \in \mathcal{X} \Rightarrow x \in \mathcal{Y})$ " — assim, por exemplo, Card $\subseteq$ Ord - e " $x \in \mathcal{X} \wedge x \in \mathcal{Y}$ ". Ainda, sendo $\mathcal{X}$ e $\mathcal{Y}$ classes, por uma função-classe $F: \mathcal{X} \rightarrow \mathcal{Y}$ entenda-se uma maneira de associar a cada $x \in \mathcal{X}$ um único $y \in \mathcal{Y}$, o qual será designado por $F(x)$.

Dados um cardinal $\kappa$ e um conjunto $X$, utilizaremos as notações

$$
\begin{aligned}
{[X]^{\kappa} } & =\{A \subseteq X|| A \mid=\kappa\}, \\
{[X]^{<\kappa} } & =\{A \subseteq X|| A \mid<\kappa\} \\
\mathrm{e}[X]^{\leq \kappa} & =\{A \subseteq X|| A \mid \leq \kappa\} .
\end{aligned}
$$

Um conjunto $X$ é dito enumerável se, e somente se, $|X| \leq \omega$.

Dados conjuntos $a$ e $b$, o par ordenado de $a$ e $b$ é o conjunto $(a, b)=\{\{a\},\{a, b\}\}$.

Dados conjuntos $X$ e $Y$, denotaremos o conjunto das funções de $X$ em $Y$ por ${ }^{X} Y$. Dados $f \in{ }^{X} Y$, um conjunto $A \subseteq X$ e um conjunto $B \subseteq Y$, definimos a restrição de $f$ a $A$ como sendo a função $f \uparrow A: A \rightarrow Y$ definida por $(f \uparrow A)(a)=f(a)$ para todo $a \in A$, a imagem direta de $A$ por $f$ como sendo o conjunto $f[A]=\{f(a) \mid a \in A\}$ e a imagem inversa de $B$ por

\footnotetext{
${ }^{2}$ Desta forma, em todas as ocorrências do termo "consistente" neste trabalho, entenda-se "consistente com ZFC".

${ }^{3} \mathrm{E}$ pertinente salientar que, embora não sejam um objeto de ZFC, as classes aqui consideradas são passíveis de serem introduzidas em ZFC - como fórmulas - de modo a satisfazer nossos propósitos; para uma breve discussão sobre esta questão, vide, e.g., [25], capítulo I, §9.
} 
$f$ como sendo o conjunto $f^{-1}[B]=\{x \in X \mid f(x) \in B\}$. Denotaremos ainda o domínio de $f$ por $\operatorname{dom}(f)=X$.

A notação $a:=b$ significará que $a$ é definido como sendo igual a $b$.

\subsubsection{Ordinais}

Na terminologia utilizada nesta dissertação, um ordinal $\alpha$ é dito

- um ordinal sucessor se, e somente se, existe um ordinal $\beta$ tal que $\alpha=\beta+1$;

· um ordinal limite se, e somente se, $\alpha$ não é um ordinal sucessor.

Da mesma maneira, um cardinal $\kappa$ é dito

- um cardinal sucessor se, e somente se, existe um cardinal $\lambda$ tal que $\kappa=\lambda^{+} ;^{4}$

· um cardinal limite se, e somente se, $\kappa$ não é um cardinal sucessor.

Dizemos ainda que um cardinal $\kappa>0$ é um cardinal limite forte se, e somente se, para todo cardinal $\lambda<\kappa$ tem-se que $2^{\lambda}<\kappa$.

Fixada uma boa-ordem $\triangleleft$ sobre um conjunto $A$, denotaremos por $t p(A)$ o tipo-de-ordem de $A$, definido como sendo o — único — ordinal tal que as ordens $\left(t p(A), \in_{t p(A)}\right)$ e $(A, \triangleleft)$ são isomorfas ${ }^{5}$. Seja então $A=\left\{a_{\xi} \mid \xi \in t p(A)\right\}$, com $a_{\xi_{1}} \triangleleft a_{\xi_{2}}$ para quaisquer $\xi_{1}, \xi_{2} \in t p(A)$ com $\xi_{1} \in \xi_{2}$; utilizaremos a notação $\lim (A)=\left\{a_{\xi} \mid \xi \in t p(A) \backslash\{0\}\right.$ e $\xi$ é um ordinal limite $\}$.

Para as próximas definições, considere um ordinal $\alpha$ fixado.

\footnotetext{
${ }^{4}$ Dado um cardinal $\lambda$, define-se $\lambda^{+}=\min \left\{\theta \leq 2^{\lambda} \mid \theta \in\right.$ Card e $\left.\theta>\lambda\right\}$.

${ }^{5}$ Sendo $X$ um conjunto, consideramos $\in_{X}=\{(x, y) \in X \times X \mid x \in y\}$.
} 
Um conjunto $F \subseteq \alpha$ é dito fechado em $\alpha$ se, e somente se, para todo ordinal limite $\gamma \in \alpha$ tem-se que $\sup (F \cap \gamma)=\gamma$ implica $\gamma \in F$.

Dizemos ainda que $D \subseteq \alpha$ é cofinal $^{6}$ em $\alpha$ se, e somente se, para todo $\beta \in \alpha$ existe $\gamma \in D$ tal que $\gamma \geq \beta$. A cofinalidade de um ordinal $\alpha$ é $c f(\alpha)=\min \{t p(A) \mid A \subseteq \alpha$ é cofinal em $\alpha\}$ - desta definição, prova-se sem grandes dificuldades que $c f(\alpha)$ é um cardinal. Um cardinal $\kappa$ é dito regular se, e somente se, $c f(\kappa)=\kappa$, e é dito singular se, e somente se, não é regular ${ }^{7}$. Um cardinal limite forte regular e não-enumerável é dito fortemente inacessivel.

Considere agora o caso em que $\alpha$ é um ordinal limite. Dizemos que $A \subseteq \alpha$ é ilimitado ${ }^{8} \mathrm{em}$ $\alpha$ se, e somente se, $\sup A=\alpha$. Dizemos ainda que $C \subseteq \alpha$ é c.u.b. em $\alpha$ se, e somente se, $C$ é fechado e ilimitado ${ }^{9}$ em $\alpha$. Finalmente, dizemos que $S \subseteq \alpha$ é estacionário em $\alpha$ se, e somente se, $\forall C \subseteq \alpha(C$ é c.u.b. em $\alpha \Rightarrow C \cap S \neq \emptyset)$.

Uma das principais aplicações do conceito de conjunto estacionário se dá por meio do seguinte resultado - cuja demonstração pode ser encontrada, por exemplo, em [25], capítulo II, lema 6.15 -

Lema (pressing-down). Sejam $\kappa$ um cardinal regular não-enumerável, $S \subseteq \kappa$ estacionário em $\kappa$ e $f: S \rightarrow \kappa$ tal que $f(\alpha)<\alpha$ para todo $\alpha \in S \backslash\{0\}$. Então existe $\gamma \in \kappa$ tal que $f^{-1}[\{\gamma\}] \subseteq S$ é estacionário em $\kappa$.

\subsubsection{O princípio $\square_{\kappa}$}

Sejam $\kappa$ um cardinal infinito e $A \subseteq \kappa^{+}$. Denotamos por $\square_{\kappa}(A)$ a afirmação:

"Existe uma seqüência $\left(C_{\alpha}\right)_{\alpha \in \lim \left(\kappa^{+}\right)}$tal que, para todo $\alpha \in \lim \left(\kappa^{+}\right)$,

\footnotetext{
${ }^{6}$ De modo mais geral, podemos definir o seguinte conceito: $D \subseteq \alpha$ é cofinal em $E \subseteq \alpha$ se, e somente se, $D \subseteq E$ e, para todo $\beta \in E$, existe $\gamma \in D$ tal que $\gamma \geq \beta$.

${ }^{7} \hat{E}$ pertinente ressaltar que todo cardinal sucessor infinito é um cardinal regular.

${ }^{8}$ Cabe notar que, se $\alpha$ é um ordinal limite, então um subconjunto de $\alpha$ é ilimitado em $\alpha$ se, e somente se, é cofinal em $\alpha$.

${ }^{9}$ A sigla c.u.b. vem do inglês closed unbounded.
} 
(i) $C_{\alpha}$ é c.u.b. em $\alpha$;

(ii) se $c f(\alpha)<\kappa$, então $\left|C_{\alpha}\right|<\kappa$;

(iii) para todo $\gamma \in \lim \left(C_{\alpha}\right)$, tem-se que $\gamma \notin A$ e $C_{\gamma}=\gamma \cap C_{\alpha}{ }^{\prime \prime}$.

Uma seqüência $\left(C_{\alpha}\right)_{\alpha \in \lim \left(\kappa^{+}\right)}$satisfazendo tais condições é dita uma $\square_{\kappa}(A)$-seqüência.

O seguinte resultado foi demonstrado em [21]:

Teorema 1.1.1. Assumindo-se $V=L$, existe $E \subseteq\left\{\alpha \in \omega_{2} \mid c f(\alpha)=\omega\right\}$ estacionário em $\omega_{2}$ para o qual $\square_{\omega_{1}}(E)$ é verdadeira.

\subsubsection{Submodelos elementares}

Nesta seção — que tem por referências básicas [20], [12] e [5] —, introduziremos o conceito de submodelo elementar e mostraremos diversos lemas sobre este tópico que serão utilizados ao longo desta dissertação. Para tanto, é necessário definir, antes de mais nada, algumas notações de lógica.

Definição 1.1.2. Seja $M$ um conjunto não-vazio. Para cada fórmula ${ }^{10} \varphi$, a relativização de $\varphi$ a $M$ é a fórmula $\varphi^{M}$, definida recursivamente por:

- Se $\varphi$ é uma fórmula atômica ${ }^{11}$, então $\varphi^{M}$ é a fórmula $\varphi$;

- $(\neg \varphi)^{M}$ é a fórmula $\neg\left(\varphi^{M}\right)$ para toda fórmula $\varphi$;

- $\left(\varphi \wedge \varphi^{\prime}\right)^{M}$ é a fórmula $\left(\varphi^{M}\right) \wedge\left(\varphi^{M}\right)$ para quaisquer fórmulas $\varphi$ e $\varphi^{\prime}$;

\footnotetext{
${ }^{10}$ Por "fórmula" entenda-se "fórmula da linguagem da teoria dos conjuntos". Cabe aqui observar que, ao enunciarmos resultados de lógica, escreveremos fórmulas usando símbolos da linguagem; porém, ao utilizarmos tais resultados - por exemplo, com aplicações à topologia -, escreveremos as mesmas de forma mais livre, com o intuito de tornar o texto mais fluido. Em todo caso, somente escreveremos uma fórmula em português quando for claro que a mesma pode ser expressa com símbolos da linguagem de teoria dos conjuntos.

${ }^{11} \mathrm{Ou}$ seja, é do tipo " $x_{1}=x_{2}$ " ou " $x_{1} \in x_{2}$ ".
} 
- $(\exists x(\varphi))^{M}$ é a fórmula $\exists x\left(x \in M \wedge\left(\varphi^{M}\right)\right)$ para toda fórmula $\varphi$ e toda variável $x$.

Dados $a_{1}, \ldots, a_{n} \in M$ e uma fórmula $\varphi\left(x_{1}, \ldots, x_{n}\right)$ - sendo que esta notação ${ }^{12}$ indica que $x_{1}, \ldots, x_{n}$ são as distintas variáveis livres de $\varphi-$, escreveremos $M \models \varphi\left[a_{1}, \ldots, a_{n}\right]$ se, e somente se, $\varphi^{M}$ é válida ao substituirmos $x_{i}$ por $a_{i}$ para cada $i \in\{1, \ldots, n\}$.

Podemos, agora, enunciar a definição de submodelo elementar.

Definição 1.1.3. Sejam $M$ e $N$ conjuntos tais que $M \subseteq N$. Dizemos que $M$ é um submodelo elementar de $N$ - e utilizamos o símbolo $M \prec N$ para denotar este fato - se, e somente se, para toda fórmula $\varphi\left(x_{1}, \ldots, x_{n}\right)$ e para $a_{1}, \ldots, a_{n} \in M$ quaisquer, tem-se que

$$
\left(M \models \varphi\left[a_{1}, \ldots, a_{n}\right]\right) \text { se, e somente se, }\left(N \models \varphi\left[a_{1}, \ldots, a_{n}\right]\right) \text {. }
$$

Essencialmente, dizer que $M \subseteq N$ é um submodelo elementar de $N$ significa, grosso modo, que uma afirmação qualquer expressa em termos de elementos de $M$ é verdadeira em $M$ se, e somente se, é verdadeira em $N$.

O seguinte resultado é uma caracterização de submodelo elementar que será muito recorrente nesta dissertação. Antes de enunciá-lo, consideremos a seguinte notação: dada uma fórmula $\varphi\left(x_{1}, \ldots, x_{n}, x\right)$, denotaremos a fórmula $\exists x\left(\varphi\left(x_{1}, \ldots, x_{n}, x\right)\right)$ por $\varphi_{x}\left(x_{1}, \ldots, x_{n}\right)$.

Lema (critério de Tarski). Sejam $M$ e $N$ conjuntos com $M \subseteq N$. Então $M \prec N$ se, $e$ somente se, para toda fórmula $\varphi\left(x_{1}, \ldots, x_{n}, x\right)$ e para $a_{1}, \ldots, a_{n} \in M$ quaisquer, tem-se que $N \models \varphi_{x}\left[a_{1}, \ldots, a_{n}\right]$ implica que existe $a \in M$ tal que $N \models \varphi\left[a_{1}, \ldots, a_{n}, a\right]$.

Demonstração. Assuma que $M \prec N$. Fixe $\varphi\left(x_{1}, \ldots, x_{n}, x\right)$ e $a_{1}, \ldots, a_{n} \in M$ arbitrários, e suponha que $N \models \varphi_{x}\left[a_{1}, \ldots, a_{n}\right]$. Como $M \prec N$, então $M \models \varphi_{x}\left[a_{1}, \ldots, a_{n}\right]$, de modo que, pela definição de $\varphi_{x}$, existe $a \in M$ tal que $M \models \varphi\left[a_{1}, \ldots, a_{n}, a\right]$. Para tal $a$, tem-se então que

\footnotetext{
${ }^{12}$ Tal notação será utilizada diversas vezes ao longo do texto.
} 
$N \models \varphi\left[a_{1}, \ldots, a_{n}, a\right]$ — novamente pela definição de $M \prec N-$, logo existe $a \in M$ tal que $N \models \varphi\left[a_{1}, \ldots, a_{n}, a\right]$.

Reciprocamente, suponha que a segunda condição do enunciado seja válida. Devemos mostrar que, para toda fórmula $\varphi\left(x_{1}, \ldots, x_{n}\right)$ e para $a_{1}, \ldots, a_{n} \in M$ quaisquer, $M \models \varphi\left[a_{1}, \ldots, a_{n}\right]$ se, e somente se, $N \models \varphi\left[a_{1}, \ldots, a_{n}\right]$. Para tanto, procederemos por indução no comprimento de fórmula.

- Se $\varphi$ é uma fórmula atômica, o resultado segue diretamente da definição do símbolo $\models$.

- Suponha que a equivalência seja válida para as fórmulas $\psi$ e $\psi^{\prime}$. Se $\varphi$ é $\neg \psi$ ou é $\psi \wedge \psi^{\prime}$, o resultado também segue diretamente da definição de $\models$.

- Suponha que a equivalência seja válida para a fórmula $\psi\left(x_{1}, \ldots, x_{n}, x\right)$ e que $\varphi\left(x_{1}, \ldots, x_{n}\right)$ seja do tipo $\exists x\left(\psi\left(x_{1}, \ldots, x_{n}, x\right)\right)$ 一 ou, o que é equivalente, suponha que $\varphi$ seja $\psi_{x}$.

Se $a_{1}, \ldots, a_{n} \in M$ são tais que $M \models \psi_{x}\left[a_{1}, \ldots, a_{n}\right]$, tem-se, das definições de $\psi_{x}$ e de $\models$, que existe $a \in M$ satisfazendo $M \models \psi\left[a_{1}, \ldots, a_{n}, a\right]$. Segue então da hipótese de indução e de $M \subseteq N$ que existe $a \in N$ tal que $N \models \psi\left[a_{1}, \ldots, a_{n}, a\right]$; logo, novamente pelas definições de $\models$ e de $\psi_{x}$, tem-se que $N \models \psi_{x}\left[a_{1}, \ldots, a_{n}\right]$.

Reciprocamente, tome $a_{1}, \ldots, a_{n} \in M$ arbitrários e assuma que $N \models \psi_{x}\left[a_{1}, \ldots, a_{n}\right]$. Segue da hipótese do teorema que existe $a \in M$ tal que $N \models \psi\left[a_{1}, \ldots, a_{n}, a\right]$, e a hipótese de indução implica então que existe $a \in M$ verificando $M \models \psi\left[a_{1}, \ldots, a_{n}, a\right]$; portanto, $M \models \psi_{x}\left[a_{1}, \ldots, a_{n}\right]$.

q.e.d.

Após ter definido o conceito de submodelo elementar - e apresentar uma caracterização útil do mesmo - , faz-se importante garantir que submodelos elementares de fato existem. O próximo teorema é a base de todas as construções de submodelos elementares que faremos, e permite que os obtenhamos tendo controle sobre sua cardinalidade. 
Teorema (Löwenheim-Skolem). Sejam $A$ e $N$ conjuntos tais que $A \subseteq N$. Então existe $M \prec N$ tal que $A \subseteq M$ e $|M| \leq|A| \cdot \omega$.

Antes de começar a demonstração, apresentemos uma definição que será necessária na mesma:

Sejam $S$ um conjunto, $\varphi\left(x_{1}, \ldots, x_{n}, x\right)$ uma fórmula e $f: S^{n} \rightarrow S$. Dizemos que $f$ é uma função de Skolem para $\varphi$ em $S$ se, e somente se, para quaisquer $a_{1}, \ldots, a_{n} \in S$, tem-se que $S \models \varphi_{x}\left[a_{1}, \ldots, a_{n}\right]$ implica que $S \models \varphi\left[a_{1}, \ldots, a_{n}, f\left(a_{1}, \ldots, a_{n}\right)\right]$.

Demonstração do teorema de Löwenheim-Skolem. Como a linguagem considerada é enumerável, há uma quantidade enumerável de fórmulas. Sejam então $\varphi_{i}$, com $i \in \omega$, as fórmulas da linguagem. Para cada $i \in \omega$, fixe, usando o axioma da escolha, uma função de Skolem $h_{i}$ para $\varphi_{i}$ em $N$.

Defina, recursivamente, $\left(M_{n}\right)_{n \in \omega}$ por $M_{0}=A$ e, para todo $n \in \omega$,

$$
M_{n+1}=M_{n} \cup\left\{h_{i}\left(a_{1}, \ldots, a_{k_{i}}\right) \mid i \in \omega, \operatorname{dom}\left(h_{i}\right)=N^{k_{i}} \text { e } a_{1}, \ldots, a_{k_{i}} \in M_{n}\right\}
$$

Afirmamos que $M=\bigcup\left\{M_{n} \mid n \in \omega\right\} \supseteq A$ é fechado com respeito a $\left\{h_{i} \mid i \in \omega\right\}-$ e, portanto, pelo critério de Tarski, é um submodelo elementar de $N$. De fato, fixe $i \in \omega$ arbitrário e, sendo $\operatorname{dom}\left(h_{i}\right)=N^{k_{i}}$, tome $a_{1}, \ldots, a_{k_{i}} \in M$ quaisquer. Como $\left\{a_{1}, \ldots, a_{k_{i}}\right\}$ é finito e $\left\{M_{n} \mid n \in \omega\right\}$ é uma cadeia ${ }^{13}$ com respeito à ordem $\subseteq$, existe $m \in \omega$ tal que $a_{1}, \ldots, a_{k_{i}} \in M_{m}$. Então, por construção, $h_{i}\left(a_{1}, \ldots, a_{k_{i}}\right) \in M_{m+1} \subseteq M$, como desejado.

Finalmente, $|M| \leq|A| \cdot \omega$ segue do fato de que, por indução em $n$, tem-se $\left|M_{n}\right| \leq|A| \cdot \omega$ para todo $n \in \omega$. q.e.d.

Note que o teorema de Löwenheim-Skolem nos permite, a partir de um conjunto $N$ dado, obter um submodelo elementar de $N$. É pertinente, assim, perguntar qual conjunto $N$ será interessante tomarmos.

\footnotetext{
${ }^{13}$ Dada uma ordem parcial $\unlhd$ sobre um conjunto $T$, dizemos que $C \subseteq T$ é uma cadeia se, e somente se, $C$ é totalmente ordenado por $\unlhd$.
} 
À luz do comentário feito logo após a definição de submodelo elementar, é natural que busquemos um conjunto $N$ no qual sejam válidas todas as afirmações que gostaríamos que fossem válidas - em particular, ZFC. Porém, segue do segundo teorema da incompletude de Gödel que não se pode obter um conjunto - em ZFC — que seja modelo para ZFC — i.e., um conjunto $N$ tal que, para todo axioma $\varphi$ de ZFC, tenha-se $N \models \varphi$. No entanto, é possível satisfazer os nossos propósitos com um pouco menos que isso, como veremos agora.

Dado um conjunto $A$, definimos o fecho transitivo de $A$ por $\operatorname{trcl}(A)=\bigcap\left\{T^{\prime} \in \wp(T) \mid T^{\prime}\right.$ é transitivo e $\left.A \subseteq T^{\prime}\right\}$, sendo $T$ um conjunto transitivo tal que $A \subseteq T$. Definimos então, para cada cardinal infinito $\theta$, o conjunto $H_{\theta}=\{A|| \operatorname{trcl}(A) \mid<\theta\}$. Nestas condições, temos que, se $\theta$ é um cardinal regular e não-enumerável, então $H_{\theta}$ é um modelo para ZFC-P — i.e., ZFC excetuando-se o axioma do conjunto das partes. ${ }^{14}$

Naturalmente, não podemos abdicar por completo do axioma do conjunto das partes; ao realizarmos uma demonstração, eventualmente necessitaremos tomar o conjunto $\wp(A)$ para algum conjunto $A$ que ocorra na mesma. No entanto, se tomarmos um cardinal $\theta$ regular e não-enumerável tal que $\wp(A) \in H_{\theta}$, podemos considerar que, particularmente para o conjunto $A$ em questão, o axioma do conjunto das partes é válido em $H_{\theta}$ - cabe notar que, se $\wp(A) \in H_{\theta}$, então $A \in H_{\theta}$.

É claro que uma demonstração, em princípio, pode exigir que o axioma do conjunto das partes seja usado várias - até mesmo infinitas — vezes. Porém, na prática, a demonstração de um resultado em topologia não requer o uso do axioma do conjunto das partes além do que um conjunto $H_{\theta}$, para $\theta$ suficientemente grande, é capaz de satisfazer. Suponha, por exemplo, que queiramos demonstrar uma afirmação sobre um certo espaço topológico $(X, \tau)$. Se quisermos tomar um conjunto $H_{\theta}$ que cumpra o papel de universo para a demonstração, podemos exigir

\footnotetext{
${ }^{14}$ Para uma demonstração deste fato, além de maiores detalhes sobre a existência, em ZFC, de $H_{\theta}$ e do fecho transitivo de conjuntos - o que não se inclui nos propósitos deste texto —, vide [25], capítulo IV, §6.

É interessante observar ainda que, se $\theta$ é um cardinal fortemente inacessível, então $H_{\theta}$ é um modelo para ZFC — vide, e.g., teorema 6.6 na mesma referência. Em virtude do segundo teorema da incompletude de Gödel, disto decorre então que, a menos que ZFC seja inconsistente, não é possível provar - em ZFC — que a afirmação "existe um cardinal fortemente inacessível" é consistente com ZFC — vide [25], capítulo IV, §10.
} 
que $\wp(X) \in H_{\theta}$ - para nos referirmos, e.g., ao conjunto de todos os subconjuntos discretos de $X$-, ou ainda que $\wp(\wp(X)) \in H_{\theta}$ - para nos referirmos, e.g., ao conjunto de todos os recobrimentos de $(X, \tau)$-; em todo caso, podemos afirmar com segurança que, se $\kappa>\omega$ é um cardinal limite forte tal que $|X|<\kappa$, então o conjunto $H_{\theta}$, com $\theta=\kappa^{+}$, pode ser tomado como universo da demonstração - no sentido de que, se tivermos necessidade do conjunto $\wp(A)$ para algum conjunto $A$ que ocorre na demonstração, então $\wp(A) \in H_{\theta} \cdot{ }^{15}$

Assim sendo, deste ponto em diante, sempre que escrevermos $H_{\theta}$, estaremos assumindo a hipótese adicional de que $\theta$ é um cardinal regular, não-enumerável e grande o suficiente para ser passível de ser tomado como universo da demonstração em questão.

Seria natural perguntarmos se um submodelo elementar de $H_{\theta}$ é, necessariamente, transitivo. A resposta é negativa; para verificar esse fato, basta aplicar o teorema de LöwenheimSkolem com $N=H_{\theta}$ e $A=\left\{\omega_{1}\right\}$. No entanto, $M$ pode ser transitivo com respeito a elementos de cardinalidade limitada, como se vê pelo lema a seguir.

Lema 1.1.4. Sejam $\kappa$ um cardinal e $M \prec H_{\theta}$ tais que $\kappa \in M$ e $\kappa \subseteq M$. Então $\forall A \in$ $M(|A| \leq \kappa \Rightarrow A \subseteq M)$.

Demonstração. Tome $A \in M \operatorname{com}|A| \leq \kappa$. Temos que $H_{\theta} \models$ " $\exists f: \kappa \rightarrow A$ sobrejetora"; logo, como $\kappa, A \in M$, segue do critério de Tarski que existe $f \in M$ tal que $H_{\theta} \models$ " $f$ é uma função sobrejetora de $\kappa \mathrm{em} A$ ". Fixe $f$ nessas condições e tome agora $a \in A$ arbitrário; como $f$ é sobrejetora, existe $\alpha \in \kappa$ tal que $a=f(\alpha)$. Então $H_{\theta} \models \exists x((\alpha, x) \in f)$; como $\alpha \in \kappa \subseteq M$, então existe $x \in M$ tal que $H_{\theta} \models(\alpha, x) \in f$ - novamente pelo critério de Tarski. Segue então que $a=x \in M$, pois $f$ é uma função.

q.e.d.

Corolário 1.1.5. Seja $M \prec H_{\theta}$. Se $A \in M$ é enumerável, então $A \subseteq M$.

Demonstração. Pelo lema anterior, basta mostrar que $\omega \in M$ e que $\omega \subseteq M$.

\footnotetext{
${ }^{15}$ Note que, para efeito de simplificar a demonstração, sempre podemos supor que $X=|X|$.
} 
Provemos que $\omega \subseteq M$; para tanto, provaremos que $M$ é um conjunto indutivo ${ }^{16}$. Temos, do axioma da existência do conjunto vazio, que

$$
H_{\theta} \models \exists v \forall x(x \notin v) ;
$$

logo, pelo critério de Tarski, existe $v \in M$ tal que

$$
H_{\theta} \models \forall x(x \notin v)
$$

e, da unicidade do conjunto vazio - garantida pelo axioma da extensionalidade -, segue que $\emptyset=v \in M$. Seja agora $x \in M$. Como o axioma do par e o axioma da união são válidos em $H_{\theta}$, tem-se — utilizando uma notação mais abreviada — que

$$
H_{\theta} \models \exists s(s=x \cup\{x\}) ;
$$

assim, segue novamente — de forma análoga ao caso anterior — do critério de Tarski e do axioma da extensionalidade que $x \cup\{x\} \in M$. Portanto, $M$ é indutivo.

Provemos agora que $\omega \in M$. Como o axioma do infinito é válido em $H_{\theta}$ e $M \prec H_{\theta}$, segue do critério de Tarski que existe um conjunto indutivo $I \in M$. Conforme observamos anteriormente, podemos assumir que $\wp(I) \in H_{\theta}$, e segue então — por aplicações sucessivas do critério de Tarski e dos axiomas da extensionalidade e da compreensão - que $\wp(I) \in M$, que $\mathcal{J}=\{J \in \wp(I) \mid J$ é indutivo $\} \in M$ e que $\bigcap \mathcal{J} \in M$. Mas $\omega=\bigcap \mathcal{J}$ - como observado na nota de rodapé de número 16 deste capítulo - , de sorte que $\omega \in M$.

q.e.d.

Procedamos agora às construções de submodelos elementares de $H_{\theta}$ que utilizaremos nesta dissertação. Note que, ao obtermos um submodelo elementar $M$ de $H_{\theta}$ utilizando apenas o

\footnotetext{
${ }^{16} \mathrm{Um}$ conjunto $I$ é dito indutivo se, e somente se, $\emptyset \in I$ e, para todo $x \in I$, tem-se que $x \cup\{x\} \in I$. Lembramos que o axioma do infinito é a afirmação "existe um conjunto indutivo" e que, sendo então $I$ um conjunto indutivo, define-se $\omega=\bigcap\{J \in \wp(I) \mid J$ é indutivo $\}$. Tem-se que a intersecção de uma família de conjuntos indutivos é também um conjunto indutivo; assim, segue que $\omega$ é indutivo e que $\omega \subseteq I^{\prime}$ para todo conjunto indutivo $I^{\prime}$. Isto implica, em particular, que a definição de $\omega$ independe do conjunto indutivo $I$ tomado, i.e., que $\omega=\bigcap\left\{J \in \wp\left(I^{\prime}\right) \mid J\right.$ é indutivo $\}$ para qualquer conjunto indutivo $I^{\prime}$.
} 
teorema de Löwenheim-Skolem na forma em que o enunciamos, não conhecemos, a princípio, muitas propriedades de $M$. As construções que se seguem nos permitem obter submodelos elementares de $H_{\theta}$ de modo a satisfazer certas condições que podem ser de nosso interesse. Para tanto, é necessário introduzir mais um conceito.

Definição 1.1.6. Dizemos que $\mathcal{M} \neq \emptyset$ é uma cadeia elementar de $H_{\theta}$ se, e somente se, $\mathcal{M}$ é uma cadeia com respeito à ordem $\subseteq$ e $M \prec H_{\theta}$ para todo $M \in \mathcal{M}$. Caso $\mathcal{M}$ seja bem-ordenada ${ }^{17}$ - digamos, $\mathcal{M}=\left\{M_{\alpha} \mid \alpha \in \sigma\right\}-$, dizemos ainda que $\mathcal{M}$ é

- uma $\epsilon$-cadeia se, e somente se, $M_{\alpha} \in M_{\alpha+1}$ para todo ordinal $\alpha$ tal que $\alpha+1 \in \sigma$.

- contínua se, e somente se, $M_{\beta}=\bigcup\left\{M_{\alpha} \mid \alpha \in \beta\right\}$ para todo ordinal limite $\beta \in \sigma$.

É válido mencionar uma caracterização que é muitas vezes utilizada como definição de cadeia elementar, a qual se baseia no seguinte fato:

Proposição 1.1.7. Se $M \prec N$ e $N \prec P$, então $M \prec P$.

Demonstração. Aplicaremos o critério de Tarski. Sejam $\varphi\left(x_{1}, \ldots, x_{n}, x\right)$ uma fórmula e $a_{1}$, $\ldots, a_{n} \in M$, e suponha que $P \models \varphi_{x}\left[a_{1}, \ldots, a_{n}\right]$. Como $N \prec P$, então $N \models \varphi_{x}\left[a_{1}, \ldots, a_{n}\right]$; assim, como $M \prec N$, segue do critério de Tarski que existe $a \in M$ tal que $N \models \varphi\left[a_{1}, \ldots, a_{n}, a\right]$. Fixe então um tal $a \in M$; temos que $P \models \varphi\left[a_{1}, \ldots, a_{n}, a\right]$ - uma vez que $N \prec P$ 一, logo existe $a \in M$ tal que $P \models \varphi\left[a_{1}, \ldots, a_{n}, a\right]$, como desejado. q.e.d.

Podemos agora caracterizar cadeia elementar da seguinte maneira:

Proposição 1.1.8. $\mathcal{M} \neq \emptyset$ é uma cadeia elementar de $H_{\theta}$ se, e somente se, $M \prec H_{\theta}$ para todo $M \in \mathcal{M}$ e $\mathcal{M}$ é uma cadeia com respeito à ordem $\prec$.

\footnotetext{
${ }^{17}$ Ao longo do texto, sempre que escrevermos uma cadeia elementar de $H_{\theta}$ como $\mathcal{M}=\left\{M_{\alpha} \mid \alpha \in \sigma\right\}$, estaremos assumindo que $M_{\alpha} \subseteq M_{\beta}$ para quaisquer $\alpha, \beta \in \sigma$ tais que $\alpha \in \beta$.
} 
Demonstração. Note que a recíproca decorre imediatamente da definição, uma vez que $M_{1} \subseteq$ $M_{2}$ sempre que $M_{1} \prec M_{2}$.

Seja então $\mathcal{M} \neq \emptyset$ uma cadeia elementar de $H_{\theta}$. Da definição de cadeia elementar de $H_{\theta}$, temos que $\forall M \in \mathcal{M}\left(M \prec H_{\theta}\right)$. Provemos então que $\mathcal{M}$ é uma cadeia com respeito a $\prec$.

Tome $M_{1}, M_{2} \in \mathcal{M}$ arbitrários. Como $\mathcal{M}$ é, por definição, uma cadeia com respeito a $\subseteq$, podemos supor, sem perda de generalidade, que $M_{1} \subseteq M_{2}$. Utilizaremos o critério de Tarski para provar que $M_{1} \prec M_{2}$.

Sejam $\varphi\left(x_{1}, \ldots, x_{n}, x\right)$ uma fórmula e $a_{1}, \ldots, a_{n} \in M_{1}$, e suponha que $M_{2} \models \varphi_{x}\left[a_{1}, \ldots, a_{n}\right]$. Como $M_{2} \prec H_{\theta}$, então $H_{\theta} \models \varphi_{x}\left[a_{1}, \ldots, a_{n}\right]$ e, pelo critério de Tarski - aplicado a $M_{1} \prec H_{\theta}$ - tem-se que existe $a \in M_{1}$ que satisfaz $H_{\theta} \models \varphi\left[a_{1}, \ldots, a_{n}, a\right]$. Fixando um tal $a \in M_{1} \subseteq M_{2}$ e valendo-se novamente do fato de que $M_{2} \prec H_{\theta}$, obtém-se que existe $a \in M_{1}$ tal que $M_{2} \models$ $\varphi\left[a_{1}, \ldots, a_{n}, a\right]$, como desejado.

q.e.d.

A utilização de cadeias elementares na obtenção de submodelos elementares dá-se por meio do resultado a seguir.

Lema 1.1.9. Se $\mathcal{M}$ é uma cadeia elementar infinita de $H_{\theta}$, então $\bigcup \mathcal{M} \prec H_{\theta}$.

Demonstração. Utilizaremos o critério de Tarski. Sejam $\varphi\left(x_{1}, \ldots, x_{n}, x\right)$ uma fórmula e $a_{1}$, $\ldots, a_{n} \in \bigcup \mathcal{M}$, e suponha que $H_{\theta} \models \varphi_{x}\left[a_{1}, \ldots, a_{n}\right]$. Como $\left\{a_{1}, \ldots, a_{n}\right\}$ é finito e $\mathcal{M}$ é uma cadeia com respeito à inclusão, existe $M \in \mathcal{M}$ tal que $a_{1}, \ldots, a_{n} \in M$. Por hipótese, $M \prec H_{\theta}$; logo, pelo critério de Tarski, existe $a \in M \subseteq \bigcup \mathcal{M}$ tal que $H_{\theta} \models \varphi\left[a_{1}, \ldots, a_{n}, a\right]$, e o resultado segue então da recíproca do critério de Tarski.

q.e.d.

Procedamos agora às construções. A primeira é motivada pelo interesse em determinar quando um subconjunto de $M$ é ou não um elemento de $M$. Introduziremos aqui a seguinte terminologia: dado um cardinal $\kappa$, dizemos que um conjunto $T$ é $\kappa$-fechado se, e somente se, $[T] \leq \kappa \subseteq T$. 
Lema 1.1.10. Sejam $\kappa$ um cardinal infinito $e A \subseteq H_{\theta}$ com $|A| \leq 2^{\kappa}$. Então existe $M \prec H_{\theta}$ $\kappa$-fechado tal que $A \subseteq M$ e $|M| \leq 2^{\kappa}$.

Demonstração. Construa, indutivamente, $\left(M_{\alpha}\right)_{\alpha \in \kappa^{+}}$da seguinte maneira:

$\cdot M_{0}=A$.

- Tendo construído $M_{\beta}$ para um $\beta \in \kappa^{+}$fixado, seja $M_{\beta+1} \prec H_{\theta}$ tal que $M_{\beta} \cup\left[M_{\beta}\right]^{\leq \kappa} \subseteq$ $M_{\beta+1}$ e $\left|M_{\beta+1}\right| \leq\left|M_{\beta} \cup\left[M_{\beta}\right]^{\leq \kappa}\right| \cdot \omega$ - a existência de um tal $M_{\beta+1}$ é garantida pelo teorema de Löwenheim-Skolem.

- Tendo construído $M_{\gamma}$ para todo $\gamma \in \beta$, sendo $\beta \in \kappa^{+} \backslash\{0\}$ um ordinal limite, defina $M_{\beta}=\bigcup_{\gamma \in \beta} M_{\gamma}$.

É imediato - por indução transfinita - que $\left\{M_{\alpha} \mid 0 \in \alpha \in \kappa^{+}\right\}$é uma cadeia elementar contínua de $H_{\theta}$. Considere, agora, $M=\bigcup_{\alpha \in \kappa^{+}} M_{\alpha}$. Então $A=M_{0} \subseteq M$ e, pelo lema 1.1.9, $M \prec H_{\theta}$. Afirmamos que, se $B \in[M]^{\leq \kappa}$, então existe $\alpha_{B} \in \kappa^{+}$tal que $B \subseteq M_{\alpha_{B}}$.

De fato, para cada $x \in B$, fixe $\eta_{x} \in \kappa^{+}$tal que $x \in M_{\eta_{x}}$. Como $\kappa^{+}$é um cardinal regular e $|B| \leq \kappa$, então o conjunto $\left\{\eta_{x} \mid x \in B\right\}$ é limitado em $\kappa^{+}$; assim, se tomarmos $\alpha \in \kappa^{+}$tal que $\alpha \geq \eta_{x}$ para todo $x \in B$, teremos que $B \subseteq M_{\alpha}$.

Temos então que $B \in\left[M_{\alpha}\right]^{\leq \kappa}$; logo, por construção, $B \in M_{\alpha+1} \subseteq M$. Portanto, $[M]^{\leq \kappa} \subseteq$ $M$.

Resta mostrar que $|M| \leq 2^{\kappa}$. Provaremos, por indução transfinita, que $\left|M_{\alpha}\right| \leq 2^{\kappa}$ para todo $\alpha \in \kappa^{+}$- o que concluirá a demonstração, pois implica que $|M| \leq 2^{\kappa} \cdot \kappa^{+} \leq 2^{\kappa} \cdot 2^{\kappa}=2^{\kappa}$.

- Por hipótese, $\left|M_{0}\right|=|A| \leq 2^{\kappa}$.

- Se $\left|M_{\beta}\right| \leq 2^{\kappa}$ para um certo $\beta \in \kappa^{+}$, segue que

$$
\left|\left[M_{\beta}\right]^{\leq \kappa}\right| \leq\left|M_{\beta}\right|^{\kappa} \leq\left(2^{\kappa}\right)^{\kappa}=2^{\kappa \cdot \kappa}=2^{\kappa}
$$


logo, por construção,

$$
\left|M_{\beta+1}\right| \leq\left|M_{\beta} \cup\left[M_{\beta}\right]^{\leq \kappa}\right| \cdot \omega \leq\left(\left|M_{\beta}\right|+\left|\left[M_{\beta}\right]^{\leq \kappa}\right|\right) \cdot \omega \leq\left(2^{\kappa}+2^{\kappa}\right) \cdot \omega=2^{\kappa} .
$$

- Se $\beta \in \kappa^{+} \backslash\{0\}$ é um ordinal limite e $\left|M_{\gamma}\right| \leq 2^{\kappa}$ para todo $\gamma \in \beta$, segue da construção de $M_{\beta}$ que $\left|M_{\beta}\right| \leq 2^{\kappa} \cdot|\beta| \leq 2^{\kappa} \cdot \kappa=2^{\kappa}$.

Assim, $\left|M_{\alpha}\right| \leq 2^{\kappa}$ para todo $\alpha \in \kappa^{+}$.

q.e.d.

A próxima construção - à qual recorreremos várias vezes ao longo desta dissertação - é motivada pela construção anterior. A condição " $M$ é $\kappa$-fechado" que ocorre na mesma é um tanto quanto forte, e em muitos casos é suficiente termos o seguinte:

Definição 1.1.11. Sejam $\kappa$ um cardinal infinito e $M \prec H_{\theta}$. Dizemos que $M$ tem a propriedade $\kappa$-covering - ou, simplesmente, que $M$ é $\kappa$-covering - se, e somente se, para todo $A \in[M]^{\leq \kappa}$ existe $B \in M$ tal que $A \subseteq B$ e $|B| \leq \kappa$.

Cabe ressaltar que esta definição de submodelo elementar $\kappa$-covering não é equivalente à introduzida em [26]; no entanto, a definição acima é adequada o suficiente para os nossos propósitos.

A vantagem de se trabalhar com um submodelo elementar $\kappa$-covering de $H_{\theta}$, quando tal condição bastar aos nossos propósitos, é que um tal $M \prec H_{\theta}$ pode ser obtido com uma cardinalidade menor ou igual à do caso anterior, como se vê pelo resultado a seguir.

Lema 1.1.12. Sejam $\kappa$ um cardinal infinito e $A \subseteq H_{\theta}$ satisfazendo $|A| \leq \kappa$. Então existe $M \prec H_{\theta}$ tal que $A \subseteq M,|M| \leq \kappa^{+}$e $M$ tem a propriedade $\kappa$-covering.

Demonstração. Defina, recursivamente, $\left\{M_{\alpha} \mid \alpha \in \kappa^{+}\right\}$por:

- $M_{0}=A$. 
- Tendo $M_{\beta}$ para um dado $\beta \in \kappa^{+}$, tome $M_{\beta+1} \prec H_{\theta} \operatorname{com} M_{\beta} \cup\left\{M_{\beta}\right\} \subseteq M_{\beta+1}$ e $\left|M_{\beta+1}\right| \leq$ $\left|M_{\beta} \cup\left\{M_{\beta}\right\}\right| \cdot \omega$ - conforme o teorema de Löwenheim-Skolem.

- Tendo $M_{\gamma}$ para todo $\gamma \in \beta$, sendo $\beta \in \kappa^{+} \backslash\{0\}$ um ordinal limite, defina $M_{\beta}=$ $\bigcup\left\{M_{\gamma} \mid \gamma \in \beta\right\}$.

Assim sendo, $\left\{M_{\alpha} \mid \alpha \in\right] 0, \kappa^{+}[\}$é uma $\epsilon$-cadeia elementar contínua de $H_{\theta}$. Tome, agora, $M=\bigcup\left\{M_{\alpha} \mid \alpha \in \kappa^{+}\right\}$. Temos que $A=M_{0} \subseteq M$ e que, pelo lema 1.1.9, $M \prec H_{\theta}$. Note que, da construção de $\left\{M_{\alpha} \mid \alpha \in \kappa^{+}\right\}$, segue por indução transfinita que $\left|M_{\alpha}\right| \leq \kappa$ para todo $\alpha \in \kappa^{+} ; \operatorname{assim},|M| \leq \kappa \cdot \kappa^{+}=\kappa^{+}$.

Provemos agora que $M$ tem a propriedade $\kappa$-covering. Para tanto, tome $A \in[M]^{\leq \kappa}$; como $|A| \leq \kappa$ e $\kappa^{+}$é regular, temos que $A \subseteq M_{\alpha}$ para algum $\alpha \in \kappa^{+}$- por uma argumentação análoga à utilizada na demonstração do lema 1.1.10. Assim, por construção, $M_{\alpha} \in M_{\alpha+1} \subseteq M$ é tal que $A \subseteq M_{\alpha}$ e $\left|M_{\alpha}\right| \leq \kappa$, satisfazendo a condição requerida.

q.e.d.

Finalmente, apresentaremos dois lemas que serão úteis mais adiante.

Lema 1.1.13. Sejam $\kappa$ um cardinal infinito e $M \prec H_{\theta}$ satisfazendo $\kappa \cup\{\kappa\} \subseteq M$. Então $\eta=\kappa^{+} \cap M$ é um ordinal limite.

Demonstração. Temos que $\eta$ é um ordinal, pois, para todo $\beta \in \eta=\kappa^{+} \cap M$, segue do lema 1.1.4 que $\beta \subseteq M$, $\log$ o todo $\alpha \in \beta$ é tal que $\alpha \in \kappa^{+} \cap M$. Além disso, $\eta$ é limite, pois $\eta=\delta+1 \in \kappa^{+}$implicaria $\delta \in M$, $\log \eta \eta=\delta+1 \in M$ uma vez que $M \prec H_{\theta}$ - e, portanto, $\eta \in \kappa^{+} \cap M=\eta$, o que é um absurdo.

q.e.d.

Lema 1.1.14. Sejam $\kappa$ um cardinal infinito, $M_{0}=\emptyset$ e $\left\{M_{\alpha} \mid 0<\alpha<\kappa^{+}\right\}$uma $\epsilon$-cadeia elementar contínua de $H_{\theta}$ com $\left\{\kappa, \kappa^{+}\right\} \cup \kappa \subseteq M_{1}$ e $\left|M_{\alpha}\right| \leq \kappa$ para todo $\alpha \in \kappa^{+}$. Sejam ainda $M=\bigcup\left\{M_{\alpha} \mid \alpha \in \kappa^{+}\right\}, \eta=\kappa^{+} \cap M$ e $N \prec H_{\theta}$ tal que $\left\{\kappa,\left(M_{\alpha}\right)_{\alpha \in \kappa^{+}}\right\} \cup \kappa \subseteq N$. Então

(a) $\left\{\kappa^{+} \cap M_{\alpha} \mid \alpha \in \kappa^{+}\right\}$é um subconjunto c.u.b. de $\kappa^{+}$;

(b) $M \cap N=M_{\eta}$. 
Demonstração. (a) Do lema 1.1.13, segue que $\kappa^{+} \cap M_{\alpha}$ é um ordinal para todo $\alpha \in \kappa^{+}$; logo, como $\left|M_{\alpha}\right| \leq \kappa$ para todo $\alpha \in \kappa^{+}$, tem-se que $\left\{\kappa^{+} \cap M_{\alpha} \mid \alpha \in \kappa^{+}\right\} \subseteq \kappa^{+}$.

Afirmamos que, para quaisquer $\alpha, \beta \in \kappa^{+}$tais que $\alpha \in \beta$, tem-se $M_{\alpha} \in M_{\beta}$.

Procederemos por indução transfinita em $\beta$. Se $\beta=\alpha+1$, a afirmação decorre do fato de que $\left\{M_{\alpha} \mid \alpha \in \kappa^{+}\right\}$é uma $\epsilon$-cadeia. Se $M_{\alpha} \in M_{\beta}$ para um certo $\beta \in\left[\alpha, \kappa^{+}\right.$, então $M_{\alpha} \in M_{\beta+1}$ decorre do fato de que, pelo lema 1.1.4, $M_{\beta} \subseteq M_{\beta+1}$. Já se $\beta \in\left[\alpha, \kappa^{+}[\right.$ é um ordinal limite tal que $M_{\alpha} \in M_{\delta}$ para todo $\delta \in\left[\alpha, \beta\left[\right.\right.$, basta fixar um $\delta_{0} \in[\alpha, \beta[$ arbitrário e obtém-se $M_{\alpha} \in M_{\delta_{0}} \subseteq \bigcup\left\{M_{\delta} \mid \delta \in \beta\right\}=M_{\beta}$ - uma vez que $\left\{M_{\alpha} \mid \alpha \in \kappa^{+}\right\}$ é contínua.

Assim sendo, para quaisquer $\alpha, \beta \in \kappa^{+}$, tem-se que $\alpha \in \beta$ implica $\kappa^{+} \cap M_{\alpha} \in \kappa^{+} \cap M_{\beta}$. Conseqüentemente, $\left|\left\{\kappa^{+} \cap M_{\alpha} \mid \alpha \in \kappa^{+}\right\}\right|=\kappa^{+}$e, portanto, $\left\{\kappa^{+} \cap M_{\alpha} \mid \alpha \in \kappa^{+}\right\}$é ilimitado em $\kappa^{+}$.

Finalmente, seja $\gamma \in \kappa^{+}$um ordinal limite e suponha que, para algum $\delta \in \kappa^{+}$, tenhase $\sup \left\{\kappa^{+} \cap M_{\alpha} \mid \alpha \in \delta\right\}=\gamma$. Se $\delta$ é um ordinal sucessor - digamos, $\delta=\xi+1$ 一, então $\gamma=\kappa^{+} \cap M_{\xi}$. Já se $\delta$ é um ordinal limite, então $\gamma=\bigcup\left\{\kappa^{+} \cap M_{\alpha} \mid \alpha \in\right.$ $\delta\}=\kappa^{+} \cap \bigcup\left\{M_{\alpha} \mid \alpha \in \delta\right\}=\kappa^{+} \cap M_{\delta}$, pois $\left\{M_{\alpha} \mid \alpha \in \kappa^{+}\right\}$é contínua. Portanto, $\left\{\kappa^{+} \cap M_{\alpha} \mid \alpha \in \kappa^{+}\right\}$é fechado.

(b) Para a primeira inclusão, tome $x \in M \cap N$ arbitrário. Temos que $H_{\theta} \models \exists \beta \in \kappa^{+}(x \in$ $\left.M_{\beta}\right)$; como $N \prec H_{\theta}$ e $\left\{x,\left(M_{\alpha}\right)_{\alpha \in \kappa^{+}}\right\} \subseteq N$ - e, portanto, $\kappa^{+}=\operatorname{dom}\left(\left(M_{\alpha}\right)_{\alpha \in \kappa^{+}}\right) \in N$ - , segue então do critério de Tarski ${ }^{18}$ que existe $\beta \in \kappa^{+} \cap N=\eta$ tal que $x \in M_{\beta}$. Isto implica que $x \in M_{\eta}$, pois $M_{\beta} \subseteq M_{\eta}$.

Para a outra inclusão, tome $x \in M_{\eta}$ arbitrário. Pelo lema 1.1.13, $\eta$ é um ordinal limite, $\operatorname{logo} M_{\eta}=\bigcup\left\{M_{\xi} \mid \xi \in \eta\right\}$ e, portanto, existe $\xi \in \eta=\kappa^{+} \cap N$ tal que $x \in M_{\xi}$. De $\xi \in N$

\footnotetext{
${ }^{18}$ Para tanto, basta notar que $\exists \beta \in \kappa^{+}\left(x \in M_{\beta}\right)$ é equivalente a $\exists \beta \in \kappa^{+} \exists P \in\left\{M_{\alpha} \mid \alpha \in \kappa^{+}\right\}\left((\beta, P) \in\left(M_{\alpha}\right)_{\alpha \in \kappa^{+}}\right.$e $\left.x \in P\right)$.
} 
e $\left(M_{\alpha}\right)_{\alpha \in \kappa^{+}} \in N$ segue então que $M_{\xi} \in N$; logo, pelo lema 1.1.4, $M_{\xi} \subseteq N$, de modo que $x \in N$. Já o fato de que $x \in M$ é imediato, pois $M_{\eta} \subseteq M$.

q.e.d.

\subsection{Topologia geral}

Denotaremos um espaço topológico pelo par ordenado $(X, \tau)$, sendo $\tau \subseteq \wp(X)$ uma topologia sobre um conjunto $X$. Por vezes, cometeremos o abuso de notação de escrever simplesmente $X$ ao invés de $(X, \tau)$, quando não houver risco à interpretação.

Para o restante desta seção, considere um espaço topológico $(X, \tau)$ fixado.

Consideraremos, em cada conjunto $Y \subseteq X$, a topologia de subespaço $\tau^{Y}=\{U \cap Y \mid U \in \tau\}$, a menos de menção em contrário. Nestas mesmas condições, dado $x \in X$, adotaremos a notação $\tau_{x}=\{U \in \tau \mid x \in U\}$.

\subsubsection{Definições e resultados básicos}

Dizemos que $x \in X$ é um ponto isolado de $(X, \tau)$ se, e somente se, $\{x\} \in \tau$.

Dados $x \in X$ e $A \subseteq X$, dizemos que $A$ é uma vizinhança de $x$ em $(X, \tau)$ se, e somente se, existe $U \in \tau_{x}$ tal que $U \subseteq A$.

Dado $x \in X$, um conjunto $\mathcal{V}$ é dito um sistema fundamental de vizinhanças para $x$ em $(X, \tau)$ se, e somente se, todo elemento de $\mathcal{V}$ é uma vizinhança de $x$ em $(X, \tau)$ e, para todo $U \in \tau_{x}$, existe $V \in \mathcal{V}$ tal que $V \subseteq U$. Um conjunto $\mathcal{B} \subseteq \tau$ é dito uma base de abertos ou, simplesmente, uma base — para $(X, \tau)$ se, e somente se, para todo $x \in X$ tem-se que $\mathcal{B}_{x}=\{U \in \mathcal{B} \mid x \in U\}$ é um sistema fundamental de vizinhanças para $x$ em $(X, \tau)$. Dizemos ainda que $\mathcal{U} \subseteq \wp(X)$ induz uma base - respectivamente, um sistema fundamental de vizinhanças para $y \in Y-\operatorname{em} Y \subseteq X$ se, e somente se, $\{Y \cap U \mid U \in \mathcal{U}\}$ é uma base para $\left(Y, \tau^{Y}\right)$ 
— respectivamente, um sistema fundamental de vizinhanças para $y$ em $\left(Y, \tau^{Y}\right)$.

Dados $x \in X$ e $A \subseteq X$, dizemos que $x$ é um ponto de acumulação de $A$ se, e somente se, para todo $U \in \tau_{x}$ tem-se que $(U \backslash\{x\}) \cap A \neq \emptyset$. Adotamos a notação $A^{d}=\{x \in X \mid x$ é ponto de acumulação de $A\}$ para todo $A \subseteq X$.

Dizemos ainda que $x$ é um ponto de acumulação completo de $A$ se, e somente se, todo $U \in \tau_{x}$ satisfaz $|U \cap A|=|A|$.

Dado $A \subseteq X$, o fecho — ou a aderência — de $A$ em $(X, \tau)$ é o conjunto

$$
\bar{A}=\bigcap\{F \subseteq X \mid F \text { é fechado em }(X, \tau) \text { e } A \subseteq F\} .
$$

Nestas condições, é simples verificar que $\bar{A}=A^{d} \cup A$.

Dizemos ainda que $x \in X$ é aderente a $A$ se, e somente se, $x \in \bar{A}$.

Nos casos em que $A \subseteq X \subseteq Y$, sendo $(Y, \sigma)$ um outro espaço topológico, utilizaremos, quando julgarmos haver possibilidade de dúvida na interpretação, as notações $\bar{A}^{X}$ e $\bar{A}^{Y}$ para designar, respectivamente, o fecho de $A$ em $(X, \tau)$ e o fecho de $A$ em $(Y, \sigma)$.

Dados $x \in X$ e $A \subseteq X$, dizemos que $x$ é um ponto interior de $A$ se, e somente se, existe $V \in \tau_{x}$ tal que $V \subseteq A$. O conjunto dos pontos interiores de $A$ será designado por $\operatorname{int}(A)-$ note que $\operatorname{int}(A)=\bigcup\{U \in \tau \mid U \subseteq A\} \in \tau$.

Finalmente, $(X, \tau)$ é dito separado à esquerda - respectivamente, separado à direita se, e somente se, existe uma boa-ordem $\triangleleft$ sobre $X$ tal que $\{x \in X \mid x \triangleleft a\}$ é fechado respectivamente, é aberto - em $(X, \tau)$ para todo $a \in X$.

\subsubsection{Propriedades de separação}

Dizemos que o espaço $(X, \tau)$ verifica — ou, simplesmente, é - 
- $T_{1}$ se, e somente se, $\{x\}$ é fechado em $(X, \tau)$ para todo $x \in X$

- $T_{2}$ se, e somente se, para quaisquer $x, y \in X$ distintos, existem $U, V \in \tau$ disjuntos satisfazendo $x \in U$ e $y \in V$;

- $T_{3}$ se, e somente se, para quaisquer $x \in X$ e $F \subseteq X$ fechado com $x \notin F$, existem $U, V \in \tau$ disjuntos satisfazendo $x \in U$ e $F \subseteq V$;

- $T_{3 \frac{1}{2}}$ se, e somente se, dados $x \in X$ e $F \subseteq X$ fechado quaisquer com $x \notin F$, existe uma função contínua $f: X \rightarrow[0,1]$ tal que $f(x)=0$ e $f(y)=1$ para todo $y \in F$;

- $T_{4}$ se, e somente se, dados $F, G \subseteq X$ fechados e disjuntos quaisquer, existem $U, V \in \tau$ disjuntos tais que $F \subseteq U$ e $G \subseteq V$.

Para cada $i \in\left\{1,2,3,3 \frac{1}{2}, 4\right\}$, denotaremos por $\mathcal{T}_{i}$ a classe dos espaços topológicos que verificam $T_{i}$.

Uma caracterização que utilizaremos muitas vezes ao longo deste texto - e cuja demonstração é imediata - é a seguinte:

Proposição 1.2.1. Um espaço topológico $(X, \tau)$ é $T_{3}$ se, e somente se, todo $x \in X$ admite um sistema fundamental de vizinhanças fechadas.

Dizemos ainda que $(X, \tau)$ é um espaço

- de Hausdorff se, e somente se, é $T_{2}$;

- regular se, e somente se, é $T_{3}$ e $T_{1}$;

- completamente regular se, e somente se, é $T_{3 \frac{1}{2}}$ e $T_{1}$;

- normal se, e somente se, é $T_{4}$ e $T_{1}$. 
Nestas condições, temos o seguinte resultado - cuja demonstração é trivial a menos da implicação $(v) \Rightarrow(i v)$, a qual decorre de, e.g., 1.5.11 em [8] —:

Proposição 1.2.2. Considere as seguintes afirmações sobre um espaço topológico $(X, \tau)$ arbitrário:

(i) $(X, \tau)$ é $T_{1}$

(ii) $(X, \tau)$ é $T_{2}$;

(iii) $(X, \tau)$ é regular;

(iv) $(X, \tau)$ é completamente regular;

(v) $(X, \tau)$ é normal.

Tem-se então que $(v) \Rightarrow(i v) \Rightarrow(i i i) \Rightarrow(i i) \Rightarrow(i) \cdot{ }^{19}$

É interessante ainda notar que cada uma das propriedades que ocorrem nos itens da proposição acima são hereditárias — ou seja, são válidas para todo subespaço de $(X, \tau)$ sempre que são válidas para $(X, \tau)-$, exceto a normalidade ${ }^{20}$. Dizemos, assim, que $(X, \tau)$ é hereditariamente normal se, e somente se, $\left(Y, \tau^{Y}\right)$ é normal para todo $Y \subseteq X$.

Dizemos que $\mathscr{C} \subseteq \tau$ é uma família celular em $X$ se, e somente se, quaisquer dois elementos distintos de $\mathscr{C}$ são disjuntos.

Seja agora $\kappa$ um cardinal infinito. Dizemos que $(X, \tau)$ é $\kappa$-coletivamente de Hausdorff se, e somente se, para todo $D \in[X]^{\leq \kappa}$ discreto e fechado em $(X, \tau)$ existe uma família celular

\footnotetext{
${ }^{19}$ Empregando a notação de classes, podemos enunciar esta proposição da seguinte maneira:

$$
\left(\mathcal{T}_{4} \cap \mathcal{T}_{1}\right) \subseteq\left(\mathcal{T}_{3 \frac{1}{2}} \cap \mathcal{T}_{1}\right) \subseteq\left(\mathcal{T}_{3} \cap \mathcal{T}_{1}\right) \subseteq \mathcal{T}_{2} \subseteq \mathcal{T}_{1}
$$

${ }^{20} \mathrm{O}$ que se pode afirmar neste caso é que, se $(X, \tau)$ é normal e $Y \subseteq X$ é fechado em $(X, \tau)$, então $\left(Y, \tau^{Y}\right)$ é normal.
} 
$\left\{U_{x} \mid x \in D\right\} \subseteq \tau$ tal que $x \in U_{x}$ para todo $x \in D$. Dizemos ainda que $(X, \tau)$ é coletivamente de Hausdorff se, e somente se, $(X, \tau)$ é $\kappa$-coletivamente de Hausdorff para todo cardinal infinito $\kappa$.

O seguinte fato será de grande valia no capítulo 3:

Proposição 1.2.3. Todo espaço topológico metrizável é coletivamente de Hausdorff.

Demonstração. Sejam $X$ um espaço topológico metrizável e $d: X \times X \rightarrow \mathbb{R}_{+}$uma métrica compatível com a topologia de $X$. Tome $D \subseteq X$ discreto $^{21}$ arbitrário e, para cada $x \in D$, fixe $r_{x} \in \mathbb{R}_{+}^{*}$ tal que $B_{d}\left(x, r_{x}\right) \cap D=\{x\}^{22}$

Afirmamos que $\left\{B_{d}\left(x, \frac{r_{x}}{2}\right) \mid x \in D\right\}$ é uma família celular em $X$. De fato, suponha, por absurdo, que existam $x, y \in D$ distintos tais que $B_{d}\left(x, \frac{r_{x}}{2}\right) \cap B_{d}\left(y, \frac{r_{y}}{2}\right) \neq \emptyset$; podemos supor, sem perda de generalidade, que $r_{x} \leq r_{y}$. Tomando então $z \in B_{d}\left(x, \frac{r_{x}}{2}\right) \cap B_{d}\left(y, \frac{r_{y}}{2}\right)$ arbitrário, segue que

$$
d(x, y) \leq d(x, z)+d(z, y)<\frac{r_{x}}{2}+\frac{r_{y}}{2} \leq \frac{r_{y}}{2}+\frac{r_{y}}{2}=r_{y},
$$

$\operatorname{logo} x \in B_{d}\left(y, r_{y}\right) \cap D=\{y\}$, o que é uma contradição.

q.e.d.

\subsubsection{A topologia da ordem}

Num ordinal $\sigma \neq \emptyset$, consideramos, a menos de menção em contrário, a topologia da ordem, definida como sendo a topologia em que:

- 0 é um ponto isolado;

- todo ordinal sucessor $\alpha \in \sigma$ é um ponto isolado;

- para cada ordinal limite $\beta \in \sigma$, o conjunto \{]$\alpha, \beta] \mid \alpha \in \beta\}$ é um sistema fundamental de vizinhanças abertas para $\beta$.

\footnotetext{
${ }^{21}$ Para obtermos a família celular desejada, não será necessário supor que $D$ é fechado em $(X, \tau)$.

${ }^{22}$ Utilizamos as notações $\mathbb{R}_{+}=\{r \in \mathbb{R} \mid r \geq 0\}, \mathbb{R}_{+}^{*}=\mathbb{R}_{+} \backslash\{0\}$ e, para $x \in X$ e $r \in \mathbb{R}_{+}^{*}$ arbitrários, $B_{d}(x, r)=\{a \in X \mid d(a, x)<r\}$.
} 
É um fato conhecido que $\sigma$, com esta topologia, torna-se um espaço topológico hereditariamente normal — vide, e.g., 2.7.5 em [8]. Cabe também notar que um conjunto $F \subseteq \sigma$ é fechado em $\sigma$ no sentido da seção 1.1.1 se, e somente se, é fechado na topologia da ordem de $\sigma$.

O seguinte lema será útil no capítulo 3:

Lema 1.2.4. Seja $\sigma$ um ordinal. Se $A \subseteq \sigma$ é aberto na topologia da ordem, então existe uma família $\left\{J_{i} \mid i \in \mathcal{I}\right\}$ de intervalos abertos em $\sigma$ satisfazendo:

(i) $\emptyset \notin\left\{J_{i} \mid i \in \mathcal{I}\right\}$;

(ii) $\forall i_{1}, i_{2} \in \mathcal{I}\left(i_{1} \neq i_{2} \Rightarrow J_{i_{1}} \cap J_{i_{2}}=\emptyset\right)$;

(iii) $A=\bigcup\left\{J_{i} \mid i \in \mathcal{I}\right\}$

(iv) para todo $i \in \mathcal{I}$, se $J_{i}^{\prime} \subseteq \sigma$ é um intervalo aberto tal que $J_{i} \subseteq J_{i}^{\prime} \subseteq A$, então $J_{i}=J_{i}^{\prime}$.

Demonstração. Defina a relação de equivalência $\sim$ em $A$ por

$$
\forall \alpha, \beta \in A(\alpha \sim \beta \Leftrightarrow] \alpha, \beta[\cup] \beta, \alpha[\subseteq A),
$$

e seja $\left\{J_{i} \mid i \in \mathcal{I}\right\}$ o conjunto das classes de equivalência que $\sim$ define em $A$.

É imediato que as condições $(i),(i i)$ e $(i i i)$ são satisfeitas. Resta provar que $J_{i}$ é um intervalo aberto para todo $i \in \mathcal{I}$ e que a condição $(i v)$ também é verificada.

Fixe $i \in \mathcal{I}$ arbitrário e sejam $\alpha=\min J_{i}$ e $\beta=\sup \left\{\gamma+1 \mid \gamma \in J_{i}\right\}$. Afirmamos que $\alpha$ é um ordinal sucessor ou $\alpha=0$; de fato, se $\alpha$ fosse um ordinal limite maior que 0 , existiria $\delta \in \alpha$ tal que $] \delta, \alpha[\subseteq] \delta, \alpha] \subseteq A-$ pois $\alpha \in J_{i} \subseteq A$ e $A \subseteq \sigma$ é aberto - , o que, pela definição de $\sim$, implicaria que $\delta \in J_{i}$, contradizendo a minimalidade de $\alpha$.

Defina agora

$$
\widehat{J}_{i}= \begin{cases}{[0, \beta[,} & \text { se } \alpha=0 \\ ] \eta, \beta[ & \text { se } \alpha=\eta+1\end{cases}
$$


Afirmamos que $\widehat{J}_{i}=J_{i}$ - e, portanto, $J_{i}$ é um intervalo aberto.

Seja $\delta \in J_{i}$; é imediato que $\alpha \leq \delta<\beta$, $\log \delta \in \in \widehat{J}_{i}$. Tome agora $\delta \in \widehat{J}_{i}$ arbitrário. Como $\delta<\beta=\sup \left\{\gamma+1 \mid \gamma \in J_{i}\right\}$, existe $\gamma \in J_{i}$ tal que $\delta \leq \gamma$; segue então que $\alpha \leq \delta \leq \gamma$, logo $\delta=\alpha \in J_{i}$ ou $\delta=\gamma \in J_{i}$ ou $\left.\delta \in\right] \alpha, \gamma\left[\subseteq J_{i}\right.$ - sendo que esta inclusão vem do fato de que $\alpha, \gamma \in J_{i}$ e da definição de $\sim$. Assim, $\widehat{J}_{i}=J_{i}$, como desejado.

Finalmente, fixe $i \in \mathcal{I}$ arbitrário e seja $J_{i}^{\prime} \subseteq \sigma$ um intervalo aberto tal que $J_{i} \subseteq J_{i}^{\prime} \subseteq A$. Tome $\gamma \in J_{i}^{\prime}$ arbitrário, e seja $\alpha$ um elemento qualquer de $J_{i}$. Como $] \alpha, \gamma[\cup] \gamma, \alpha\left[\subseteq J_{i}^{\prime} \subseteq A\right.$, segue da definição de $\sim$ que $\gamma \in J_{i}$. Portanto, $J_{i}=J_{i}^{\prime}$.

q.e.d.

\subsubsection{Compacidade}

Dizemos que $\mathscr{C} \subseteq \wp(X)$ é um recobrimento de $X$ se, e somente se, $\bigcup \mathscr{C}=X$. Nestas condições, um sub-recobrimento de $\mathscr{C}$ é um conjunto $\mathscr{C}^{\prime} \subseteq \mathscr{C}$ que também é um recobrimento de $X$, e um refinamento de $\mathscr{C}$ é um recobrimento $\mathscr{D}$ de $X$ tal que, para todo $A \in \mathscr{D}$, existe $B \in \mathscr{C}$ tal que $A \subseteq B$. Ainda, $\mathscr{C}$ é dito um recobrimento aberto de $X$ se, e somente se, $\mathscr{C} \subseteq \tau$; da mesma maneira, $\mathscr{D}$ é dito um refinamento aberto de $\mathscr{C}$ se, e somente se, $\mathscr{D} \subseteq \tau$.

Seja $\kappa$ um cardinal infinito. Dizemos que $(X, \tau)$ é inicialmente- $\kappa$-compacto se, e somente se, todo recobrimento aberto $\mathscr{C}$ de $X$ que satisfaz $|\mathscr{C}| \leq \kappa$ admite um sub-recobrimento finito $^{23}$. Dizemos ainda que $(X, \tau)$ é enumeravelmente compacto se, e somente se, $(X, \tau)$ é inicialmente- $\omega$-compacto.

O resultado a seguir apresenta uma caracterização interessante deste conceito.

Proposição 1.2.5. Um espaço topológico $(X, \tau)$ é inicialmente- $\kappa$-compacto se, e somente se, todo $A \in[X]^{\leq \kappa}$ infinito admite ponto de acumulação completo em $(X, \tau)$.

Demonstração. Suponha que $(X, \tau)$ é inicialmente- $\kappa$-compacto e tome $A \in[X] \leq \kappa$ infinito

\footnotetext{
${ }^{23} \mathrm{E}$ fácil ver que $(X, \tau)$ é inicialmente- $\kappa$-compacto se, e somente se, toda família não-vazia $\mathcal{F} \in[\wp(X)]^{\leq \kappa}$ de fechados em $(X, \tau)$ que possui a propriedade da intersecção finita - i.e., tal que $\bigcap \mathcal{F}^{\prime} \neq \emptyset$ para todo $\mathcal{F}^{\prime} \in[\mathcal{F}]^{<\omega} \backslash\{\emptyset\}$ - verifica $\bigcap \mathcal{F} \neq \emptyset$.
} 
arbitrário. Seja $\lambda=|A| \leq \kappa$, e considere uma ordenação $A=\left\{x_{\alpha} \mid \alpha \in \lambda\right\}-\operatorname{com} x_{\alpha} \neq x_{\beta}$ sempre que $\alpha, \beta \in \lambda$ forem distintos.

Tratemos primeiramente o caso em que $\lambda$ é um cardinal regular. Defina, para cada $\alpha \in \lambda$,

$$
S_{\alpha}=\left\{x_{\beta} \mid \beta \in[\alpha, \lambda[\}\right.
$$

Como $(X, \tau)$ é inicialmente- $\kappa$-compacto, então $\bigcap_{\alpha \in \kappa} \overline{S_{\alpha}} \neq \emptyset$ - pois $\left\{\overline{S_{\alpha}} \mid \alpha \in \kappa\right\}$ é uma família de fechados que possui a propriedade da intersecção finita. Tome então $z \in \bigcap_{\alpha \in \kappa} \overline{S_{\alpha}}$ arbitrário; afirmamos que z é um ponto de acumulação completo de $A$ em $(X, \tau)$. De fato, suponha, por absurdo, que exista $U \in \tau_{z}$ tal que $|U \cap A|<\lambda$. Por ser $\lambda$ um cardinal regular, existe então $\alpha \in \lambda$ tal que $(U \cap A) \cap S_{\alpha}=\emptyset$; mas $(U \cap A) \cap S_{\alpha}=U \cap S_{\alpha} \neq \emptyset$ - uma vez que $z \in \overline{S_{\alpha}}-$, uma contradição.

Consideremos agora o caso em que $\lambda$ é um cardinal singular. Seja $\sigma=\operatorname{cf}(\lambda)<\lambda \leq \kappa$, e tome uma seqüência crescente $\left(\lambda_{\eta}\right)_{\eta \in \sigma}$ de cardinais menores que $\lambda$ tal que $\sup _{\eta \in \sigma} \lambda_{\eta}=\lambda$; podemos assumir que cada $\lambda_{\eta}$ da seqüência é um cardinal regular, tomando, se necessário for, $\lambda_{\eta}^{+}$ao invés de $\lambda_{\eta}$. Pelo caso anterior, para cada $\eta \in \sigma$ tem-se que $\left\{x_{\alpha} \mid \alpha \in \lambda_{\eta}\right\}$ admite um ponto de acumulação completo em $(X, \tau)$ - digamos, $z_{\eta}$. Novamente pelo caso anterior, existe $z \in X$ que é ponto de acumulação completo do conjunto $\left\{z_{\eta} \mid \eta \in \sigma\right\}$ em $(X, \tau)$ - pois $\sigma=c f(\lambda)$ é um cardinal regular. Tome agora $U \in \tau_{z}$ arbitrário; provaremos que $|U \cap A|=\lambda$. Para tanto, fixe um cardinal $\mu<\lambda$ arbitrário; como $\sup _{\eta \in \sigma} \lambda_{\eta}=\lambda$, existe então $\xi \in \sigma$ tal que $\mu<\lambda_{\eta}$ para todo $\eta \in\left[\xi, \sigma\left[\right.\right.$. Tome então $\eta \in\left[\xi, \sigma\left[\right.\right.$ tal que $z_{\eta} \in U$ — um tal $\eta$ existe, pois $z$ é ponto de acumulação completo de $\left\{z_{\eta} \mid \eta \in \sigma\right\}$ em $(X, \tau)$-; como $U \in \tau_{z_{\eta}}$ e $z_{\eta}$ é ponto de acumulação completo de $\left\{x_{\alpha} \mid \alpha \in \lambda_{\eta}\right\}$ em $(X, \tau)$, segue que $|U \cap A| \geq\left|U \cap\left\{x_{\alpha} \mid \alpha \in \lambda_{\eta}\right\}\right|=\lambda_{\eta}>\mu$. O afirmado decorre então do fato de que $\mu<\lambda$ foi tomado arbitrariamente; assim, como $U \in \tau_{z}$ também foi tomado arbitrariamente, segue que $z$ é ponto de acumulação completo de $A$ em $(X, \tau)$.

Para a recíproca da proposição, suponha que $(X, \tau)$ não seja inicialmente- $\kappa$-compacto; existe então um recobrimento aberto $\mathscr{C}=\left\{U_{\alpha} \mid \alpha \in \lambda\right\}$ de $X$ tal que $\lambda \leq \kappa$ e que não admite sub-recobrimento finito. Podemos supor que $\lambda$ é o menor cardinal para o qual existe um tal 
recobrimento, e assim podemos também supor que, para cada $\alpha \in \lambda$, ocorre $U_{\alpha} \nsubseteq \bigcup_{\beta \in \alpha} U_{\beta}$ caso contrário, podemos considerar $D=\left\{\alpha \in \lambda \mid U_{\alpha} \nsubseteq \bigcup_{\beta \in \alpha} U_{\beta}\right\}$ e, pela minimalidade de $\lambda$, temos que $t p(D)=\lambda$ e que $\left\{U_{\alpha} \mid \alpha \in D\right\}$ é um recobrimento que satisfaz tais condições. Agora, para cada $\alpha \in \lambda$, tome $x_{\alpha} \in U_{\alpha} \backslash \bigcup_{\beta \in \alpha} U_{\beta}$ arbitrário; afirmamos que o conjunto $\left\{x_{\alpha} \mid \alpha \in \lambda\right\}$ não admite ponto de acumulação completo em $(X, \tau)$. De fato, suponha, por absurdo, que $z \in X$ seja ponto de acumulação completo de $\left\{x_{\alpha} \mid \alpha \in \lambda\right\}$ em $(X, \tau)$. Tome $\beta \in \lambda$ tal que $z \in U_{\beta}$; devemos ter então que $\left|U_{\beta} \cap\left\{x_{\alpha} \mid \alpha \in \lambda\right\}\right|=\lambda$. Mas, pela construção de $\left\{x_{\alpha} \mid \alpha \in \lambda\right\}$, temos que $U_{\beta} \cap\left\{x_{\alpha} \mid \alpha \in \lambda\right\} \subseteq\left\{x_{\alpha} \mid \alpha \leq \beta\right\}$, o que implica que $\left|U_{\beta} \cap\left\{x_{\alpha} \mid \alpha \in \lambda\right\}\right| \leq|\beta|<\lambda$, uma contradição.

q.e.d.

Seja agora $\mathcal{F} \subseteq \wp(X)$. Dizemos que $\mathcal{F}$ é localmente finito se, e somente se, para todo $x \in X$ existe $U_{x} \in \tau_{x}$ tal que $\left\{F \in \mathcal{F} \mid F \cap U_{x} \neq \emptyset\right\}$ é finito.

Dizemos então que $(X, \tau)$ é paracompacto se, e somente se, todo recobrimento aberto de $X$ admite um refinamento aberto que é localmente finito.

Em espaços paracompactos, algumas propriedades de separação mais fracas mostram-se equivalentes a outras mais fortes; mais precisamente, temos o seguinte resultado — vide, e.g., $5.1 .5 \mathrm{em}[8]-$ :

Teorema 1.2.6. (a) Todo espaço paracompacto e $T_{2}$ é $T_{3}$.

(b) Todo espaço paracompacto e $T_{3}$ é $T_{4}$.

A classe dos espaços topológicos compactos - i.e., os espaços topológicos que são inicialmente- $\kappa$-compactos para todo cardinal infinito $\kappa$ - será designada por $\mathcal{C}$. Adotaremos também a notação $\mathcal{C}_{2}=\mathcal{C} \cap \mathcal{T}_{2}$. Note que todo espaço topológico compacto é paracompacto; assim, é uma consequiência do teorema anterior que todo $X \in \mathcal{C}_{2}$ é normal.

Ainda, $(X, \tau)$ é dito localmente compacto se, e somente se, todo ponto de $X$ admite um sistema fundamental de vizinhanças compactas. Note que todo subconjunto compacto de um espaço de Hausdorff é fechado; assim sendo, segue da proposição 1.2.1 que todo espaço de Hausdorff localmente compacto é regular. 


\subsubsection{Metrizabilidade}

Uma família $\mathcal{F} \subseteq \wp(X)$ é dita $\sigma$-localmente finita se, e somente se, $\mathcal{F}$ é uma reunião enumerável de conjuntos localmente finitos. Dizemos ainda que $\mathcal{F}$ é pontualmente enumerável se, e somente se, $\{A \in \mathcal{F} \mid x \in A\}$ é enumerável para todo $x \in X$. Cabe notar que toda família $\sigma$-localmente finita é pontualmente enumerável, mas a recíproca não é verdadeira: a família de todos os subconjuntos unitários da reta real é pontualmente enumerável, mas não é $\sigma$-localmente finita; isto deve-se ao fato de que todo subconjunto não-enumerável da reta real admite ponto de acumulação, uma vez que a reta real tem extent enumerável — vide seção sobre funções cardinais ao final deste capítulo.

Enunciaremos agora o teorema que será nossa principal ferramenta para verificar a metrizabilidade de um espaço topológico - uma demonstração do mesmo pode ser encontrada, por exemplo, em [8], 4.4.7-:

Teorema (Nagata-Smirnov). Um espaço topológico é metrizável se, e somente se, é regular e admite uma base $\sigma$-localmente finita.

Em particular, todo espaço metrizável admite uma base pontualmente enumerável.

Do teorema de Nagata-Smirnov, obtemos o seguinte corolário:

Corolário 1.2.7. Sejam $X$ um espaço topológico e $\mathcal{A}$ uma família de abertos de $X$ dois a dois disjuntos tais que $X=\bigcup \mathcal{A}$. Suponha que todo elemento de $\mathcal{A}$ é metrizável. Então $X$ é metrizável.

Demonstração. Para cada $A \in \mathcal{A}$, fixe uma base $\mathcal{B}_{A}$ de $A$ que é $\sigma$-localmente finita. Então $\bigcup_{A \in \mathcal{A}} \mathcal{B}_{A}$ é uma base de $X$ - uma vez que todo $A \in \mathcal{A}$ é aberto em $X$ - e é $\sigma$-localmente finita devido ao fato de que $\mathcal{A}$ é uma família de abertos dois a dois disjuntos.

Quanto à regularidade de $X$, tome $A \in \mathcal{A}$ e $a \in A$ arbitrários. Por hipótese, $a$ admite um sistema fundamental de vizinhanças fechadas em $A-\operatorname{digamos}, \mathcal{V}$. Como $A$ é aberto e 
fechado em $X$, segue que $\mathcal{V}$ é um sistema fundamental de vizinhanças fechadas de $a$ em $X$. Temos então que $X$ é regular, pois, como cada $A \in \mathcal{A}$ é um espaço $T_{1}$ e é fechado em $X$, então $X$ é também um espaço $T_{1}$.

q.e.d.

\subsubsection{Um teorema de Miščenko}

O intuito desta seção é demonstrar um resultado - devido a A. Miščenko - do qual faremos uso no capítulo 3. A demonstração aqui apresentada encontra-se em [5], e consiste, essencialmente, em dois lemas que se baseiam em técnicas de submodelos elementares desenvolvidas na primeira parte deste capítulo.

Lema 1.2.8. Sejam $(X, \tau)$ um espaço topológico que admite uma base pontualmente enumerável, $\mathcal{B} \subseteq \tau$ uma base qualquer para $(X, \tau)$ e $M \prec H_{\theta} \operatorname{com}\{X, \tau, \mathcal{B}\} \subseteq M$. Então, para cada $x \in \overline{X \cap M}$, tem-se que $\mathcal{B} \cap M$ contém um sistema fundamental de vizinhanças para $x$ em $(X, \tau)$.

Demonstração. Por hipótese,

$$
H_{\theta} \models \exists \mathcal{W}(\mathcal{W} \text { é uma base pontualmente enumerável para }(X, \tau)) ;
$$

logo, como $M \prec H_{\theta}$, segue do critério de Tarski que existe $\mathcal{W} \in M$ tal que

$$
H_{\theta} \models \mathcal{W} \text { é uma base pontualmente enumerável para }(X, \tau) \text {. }
$$

Fixe agora $x \in \overline{X \cap M}$ arbitrário e $U \in \mathcal{W}$ qualquer com $x \in U$; existem então $V \in \mathcal{B}$ e $W \in \mathcal{W}$ tais que $x \in W \subseteq V \subseteq U$. Como $x \in \overline{X \cap M}$, existe $y \in W \cap(X \cap M)$. Temos que $\{\mathcal{W}, y\} \in M, \operatorname{logo}\{\Omega \in \mathcal{W} \mid y \in \Omega\} \in M$ - aplicando-se o critério de Tarski a

$$
H_{\theta} \models \exists A(\forall \Omega(\Omega \in A \Leftrightarrow(\Omega \in \mathcal{W} \text { e } y \in \Omega))) .
$$

Por hipótese, $\mathcal{W}$ é pontualmente enumerável, $\log \{\Omega \in \mathcal{W} \mid y \in \Omega\}$ é enumerável e, pelo corolário 1.1.5, $\{\Omega \in \mathcal{W} \mid y \in \Omega\} \subseteq M$. Disto decorre que $\{U, W\} \subseteq M$; assim, como 
$H_{\theta} \models \exists V \in \mathcal{B}(W \subseteq V \subseteq U)$, segue do critério de Tarski que existe $V^{\prime} \in \mathcal{B} \cap M$ tal que $W \subseteq V^{\prime} \subseteq U$. Portanto, existe $V^{\prime} \in \mathcal{B} \cap M$ tal que $x \in V^{\prime} \subseteq U$, de modo que $\mathcal{B} \cap M$ contém, de fato, um sistema fundamental de vizinhanças para $x$ em $(X, \tau)$.

q.e.d.

Lema 1.2.9. Sejam $(X, \tau) \in \mathcal{T}_{1}$ enumeravelmente compacto e $M \prec H_{\theta}$ enumerável tais que $\{X, \tau\} \subseteq M$. Suponha que $\tau \cap M$ não seja uma base para $(X, \tau)$. Então existe $x \in \overline{X \cap M}$ tal que $\tau \cap M$ não contém um sistema fundamental de vizinhanças para $x$ em $(X, \tau)$.

Demonstração. Suponha, por absurdo, que a afirmação do enunciado seja falsa. Então $\overline{X \cap M}$ $\neq X$ - do contrário, $\tau \cap M$ seria uma base para $(X, \tau)$, o que, por hipótese, não ocorre. Tome então $y \in X \backslash \overline{X \cap M}$ arbitrário. Para cada $x \in \overline{X \cap M}$, tem-se que $\tau \cap M$ contém um sistema fundamental de vizinhanças para $x$ em $(X, \tau)$; em particular, existe $U_{x} \in \tau \cap M$ tal que $x \in U_{x}$ e $y \notin U_{x}$, uma vez que $(X, \tau) \in \mathcal{T}_{1}$. Obtemos então um recobrimento $\mathscr{C}=\left\{U_{x} \mid x \in\right.$ $\overline{X \cap M}\} \subseteq \tau \cap M$ de $\overline{X \cap M}$ tal que $y \notin \bigcup \mathscr{C}$. Como $M$ é enumerável, então $\mathscr{C}$ também o é; assim, existe $\mathscr{C}^{\prime} \subseteq \mathscr{C}$ finito tal que $\overline{X \cap M} \subseteq \bigcup \mathscr{C}^{\prime}$, pois $\overline{X \cap M}$ é enumeravelmente compacto - note que $\mathscr{C}^{\prime} \in M$, pois $\mathscr{C}^{\prime} \subseteq M$ é finito. Segue então que, em particular, $X \cap M \subseteq \bigcup \mathscr{C}^{\prime}$, o que implica que $X \cap M \subseteq\left(\bigcup \mathscr{C}^{\prime}\right) \cap M$, logo $M \models X \subseteq \bigcup \mathscr{C}^{\prime}$. Assim, como $M \prec H_{\theta}$, tem-se que $H_{\theta} \models X \subseteq \bigcup \mathscr{C}^{\prime}$; mas isto é um absurdo, pois $y \notin \bigcup \mathscr{C} \supseteq \bigcup \mathscr{C}^{\prime}$.

q.e.d.

O teorema segue, então, como uma decorrência quase imediata dos dois últimos lemas.

Teorema 1.2.10. Seja $(X, \tau)$ um espaço topológico $T_{1}$ e enumeravelmente compacto que admite uma base pontualmente enumerável. Então $(X, \tau)$ admite uma base enumerável.

Demonstração. Tome $M \prec H_{\theta}$ enumerável tal que $\{X, \tau\} \subseteq M$. Pelo lema 1.2.8, para todo $x \in \overline{X \cap M}$ tem-se que $\tau \cap M$ contém um sistema fundamental de vizinhanças para $x$. Segue então do lema 1.2.9 que $\tau \cap M$ é uma base para $(X, \tau)$ e, como $M$ é enumerável, obtemos o desejado.

q.e.d.

Uma conseqüência interessante deste teorema e do teorema de Nagata-Smirnov é: 
Corolário 1.2.11. Seja $(X, \tau)$ um espaço de Hausdorff enumeravelmente compacto. Então as seguintes afirmações são equivalentes:

(a) $(X, \tau)$ é metrizável;

(b) $(X, \tau)$ admite uma base pontualmente enumerável;

(c) $(X, \tau)$ admite uma base enumerável.

Além disso, se as condições acima são válidas, então $(X, \tau)$ é compacto.

Demonstração. É imediato que $(a) \Rightarrow(b)$ - pelo teorema de Nagata-Smirnov - e $(b) \Rightarrow(c)$ - pelo teorema anterior. Suponha agora que $(X, \tau)$ admite uma base enumerável. Como $(X, \tau)$ é enumeravelmente compacto, segue então que $(X, \tau)$ é compacto e, portanto, regular — pois verifica $T_{2}$-; assim, pelo teorema de Nagata-Smirnov, $(X, \tau)$ é metrizável. $\quad$ q.e.d.

\subsubsection{Funções cardinais}

Uma função cardinal é uma função-classe $\varphi: \mathcal{D} \rightarrow$ Card tal que

(i) $\mathcal{D} \subseteq \mathcal{T}$

(ii) $\varphi(X) \geq \omega$ para todo $X \in \mathcal{D}$;

(iii) para quaisquer $X, Y \in \mathcal{D}$ homeomorfos, tem-se que $\varphi(X)=\varphi(Y)$.

Uma função cardinal $\varphi: \mathcal{D} \rightarrow$ Card é dita monótona se, e somente se, $\varphi(X) \leq \varphi(Y)$ para quaisquer $X, Y \in \mathcal{D}$ tais que $X \subseteq Y$.

Dada uma função cardinal $\varphi: \mathcal{D} \rightarrow$ Card, definimos uma nova função cardinal

$$
\begin{aligned}
h \varphi: \mathcal{D} & \rightarrow \text { Card } \\
X & \mapsto \sup \{\varphi(Y) \mid Y \in \mathcal{D} \text { e } Y \subseteq X\} .
\end{aligned}
$$


Note que, se $\varphi$ é monótona, então $h \varphi=\varphi$.

Introduziremos agora as funções cardinais que serão de nosso interesse nesta dissertação. Para tanto, fixe $X \in \mathcal{T}$ arbitrário e seja $\tau$ sua topologia.

Comecemos pelas chamadas funções cardinais globais.

O peso de $X$ é definido por

$$
w(X)=\min \{|\mathcal{B}| \mid \mathcal{B} \text { é uma base de abertos para } X\}+\omega .
$$

Note que $w$ é uma função cardinal monótona.

A densidade de $X$ é definida por

$$
d(X)=\min \{|D| \mid D \subseteq X \text { é denso em } X\}+\omega .
$$

Dizemos que $X$ é separável se, e somente se, $d(X)=\omega$.

Um resultado importante no que concerne à função cardinal $d$ é o seguinte teorema - para um demonstração do mesmo, vide, e.g., 11.2 em [17] -:

Teorema (Hewitt-Marczewski-Pondiczery). Sejam $\kappa$ um cardinal infinito, I um conjunto não-vazio com $|I| \leq 2^{\kappa}$ e $\left\{X_{i} \mid i \in I\right\}$ uma família de espaços topológicos satisfazendo $d\left(X_{i}\right) \leq \kappa$ para todo $i \in I$. Então $d\left(\prod_{i \in I} X_{i}\right) \leq \kappa$.

O grau de Lindelöf de $X$ é definido por

$$
\begin{aligned}
L(X)=\min \{\kappa \in \text { Card } \mid \text { todo recobrimento aberto de } X \text { admite um } \\
\text { sub-recobrimento de cardinalidade menor ou igual a } \kappa\}+\omega .
\end{aligned}
$$


O spread de $X$ é definido por

$$
s(X)=\sup \{|A| \mid A \subseteq X \text { é discreto }\}+\omega
$$

- e é, assim como $w$, uma função cardinal monótona.

O extent de $X$ é definido por

$$
e(X)=\sup \{|A| \mid A \subseteq X \text { é discreto e fechado em } X\}+\omega .
$$

Um fato simples de se verificar e que será utilizado diversas vezes no texto é que $A \subseteq X$ é discreto e fechado em $X$ se, e somente se, $A$ não admite ponto de acumulação em $X$.

A celularidade de $X$ é definida por

$$
c(X)=\sup \{|\mathscr{C}| \mid \mathscr{C} \text { é uma família celular em } X\}+\omega .
$$

A proposição a seguir enuncia algumas desigualdades básicas envolvendo essas funções cardinais. $^{24}$

Proposição 1.2.12. Para $X \in \mathcal{T}$ arbitrário,

(i) $e(X) \leq L(X) \leq h L(X) \leq|X|+\omega$;

(ii) $c(X) \leq d(X) \leq h d(X) \leq|X|+\omega ;$

(iii) $e(X) \leq h e(X)=s(X) \leq h L(X) \leq w(X)$;

(iv) $c(X) \leq h c(X)=s(X) \leq h d(X) \leq w(X)$.

\footnotetext{
${ }^{24} \mathrm{~A}$ demonstração de tais desigualdades foge aos propósitos desta dissertação; no entanto, os mesmos podem ser verificados sem grandes dificuldades.
} 
Passemos agora às chamadas funções cardinais locais — as quais são assim denominadas por serem primeiramente definidas em cada ponto de $X$.

O caráter de $X$ é definido por $\chi(X)=\sup \{\chi(x, X) \mid x \in X\}$, sendo

$$
\begin{aligned}
\chi(x, X)=\min \{|\mathcal{V}| & \mid \mathcal{V} \text { é um sistema fundamental } \\
& \text { de vizinhanças para } x \text { em } X\}+\omega
\end{aligned}
$$

para todo $x \in X$.

Podemos ainda definir o caráter de um subconjunto de $X$ - conceito este que será utilizado na demonstração de um resultado na seção 2.3.1. Dado $A \subseteq X$, dizemos que $V \subseteq X$ é uma vizinhança de $A$ em $X$ se, e somente se, existe $U \in \tau$ tal que $A \subseteq U \subseteq V$. Dizemos ainda que $\mathcal{V}$ é um sistema fundamental de vizinhanças para $A$ em $X$ se, e somente se, todo elemento de $\mathcal{V}$ é uma vizinhança de $A$ em $X$ e, para todo $U \in \tau$ com $A \subseteq U$, existe $V \in \mathcal{V}$ tal que $V \subseteq U$.

Assim sendo, definimos

$$
\begin{aligned}
\chi(A, X)=\min \{|\mathcal{V}| & \mid \mathcal{V} \text { é um sistema fundamental } \\
& \text { de vizinhanças para } A \text { em } X\}+\omega
\end{aligned}
$$

para todo $A \subseteq X$

Sejam $x \in X$ e $\mathcal{W} \subseteq \tau_{x}$. Dizemos que $\mathcal{W}$ é uma pseudobase para $x$ em $X$ se, e somente se, $\mathcal{W} \neq \emptyset$ e $\bigcap \mathcal{W}=\{x\}$. Note que $x$ admite uma pseudobase em $X$ se, e somente se, $\bigcap \tau_{x}=\{x\}$, o que ocorre se, e somente se, $x \notin \overline{\{y\}}$ para todo $y \in X \backslash\{x\}$. Disto segue que todo ponto de $X$ admite uma pseudobase se, e somente se, $X$ é um espaço $T_{1}$.

Suponha então que $X \in \mathcal{T}_{1}$. Definimos o pseudocaráter de $X$ por $\psi(X)=\sup \{\psi(x, X) \mid x \in$ $X\}$, sendo

$$
\psi(x, X)=\min \{|\mathcal{W}| \mid \mathcal{W} \text { é uma pseudobase para } x \text { em } X\}+\omega
$$

para todo $x \in X$. 
O tightness de $X$ é definido por $t(X)=\sup \{t(x, X) \mid x \in X\}$, sendo

$$
\begin{aligned}
t(x, X)=\min \{\kappa \in C \text { ard } \mid & \text { para todo } A \subseteq X \text { tal que } x \in \bar{A}, \\
& \text { existe } B \in[A] \leq \kappa \text { tal que } x \in \bar{B}\}+\omega
\end{aligned}
$$

para todo $x \in X$.

As propriedades destas funções de que faremos uso podem ser resumidas na seguinte proposição — cuja demonstração, por ser bastante simples, será omitida —:

Proposição 1.2.13. As funções cardinais $\chi, \psi$ e $t$ são monótonas e satisfazem

(i) $\psi(X) \leq \chi(X)$ para todo $X \in \mathcal{T}_{1}$;

(ii) $t(X) \leq \chi(X)$ para todo $X \in \mathcal{T}$.

Enunciamos ainda um resultado do qual faremos uso no capítulo 3:

Lema 1.2.14. Sejam $(X, \tau) \in \mathcal{T}_{3}, D \subseteq X$ denso em $(X, \tau), x \in D$ e $\mathcal{V}$ um sistema fundamental de vizinhanças abertas para $x$ em $D$. Então $\mathcal{U}=\{\operatorname{int}(\bar{V}) \mid V \in \mathcal{V}\}$ é um sistema fundamental de vizinhanças abertas para $x$ em $X .^{25}$

Demonstração. Tome $\Omega \in \tau$ arbitrário tal que $x \in \Omega$. Como $(X, \tau)$ satisfaz $T_{3}$, existe $\Omega^{\prime} \in \tau$ tal que $x \in \Omega^{\prime} \subseteq \overline{\Omega^{\prime}} \subseteq \Omega$. Por hipótese, existe então $V \in \mathcal{V}$ tal que $V \subseteq \Omega^{\prime} \cap D$; disto segue que $\bar{V} \subseteq \overline{\Omega^{\prime} \cap D}=\overline{\Omega^{\prime}} \subseteq \Omega$, logo $\operatorname{int}(\bar{V}) \subseteq \Omega$.

Basta agora provar que $x \in \operatorname{int}(\bar{V})$. Para tanto, tome $U \in \tau$ tal que $V=U \cap D$; como $(X, \tau) \in \mathcal{T}_{3}$, existe $U^{\prime} \in \tau$ com $x \in U^{\prime} \subseteq \overline{U^{\prime}} \subseteq U$. Temos então que $x \in U^{\prime} \subseteq \overline{U^{\prime}}=\overline{U^{\prime} \cap D} \subseteq$ $\overline{U \cap D}=\bar{V}, \operatorname{logo} x \in \operatorname{int}(\bar{V})$.

q.e.d.

\footnotetext{
${ }^{25}$ Ao escrevermos $\operatorname{int}(\bar{V})$, tanto a aderência de $V$ quanto o interior de $\bar{V}$ são considerados com respeito a $(X, \tau)$.
} 
Note que este lema implica que, se $(X, \tau) \in \mathcal{T}_{3}$ e $D \subseteq X$ é denso em $(X, \tau)$, então $\chi(x, X)=\chi(x, D)$ para todo $x \in D$.

Maiores informações sobre funções cardinais e suas propriedades podem ser encontradas, por exemplo, em [17]. 


\section{Capítulo 2}

\section{Reflexão de funções cardinais}

Nosso interesse neste capítulo — baseado principalmente em [18] — é estudar as propriedades de reflexão de funções cardinais. O principal conceito, que expressa a propriedade enunciada na introdução desta dissertação, encontra-se no item (a) da definição 2.1.1; na mesma definição, são introduzidos outros três conceitos que se relacionam a este através da proposição 2.1.2: a reflexão forte de cardinais, a propriedade de Darboux e a propriedade $I U$. Cabe salientar que, embora estes dois últimos conceitos apresentem interesse próprio, a ênfase será dada a resultados sobre reflexão e reflexão forte de cardinais - vide observação feita após a proposição 2.1.2.

\section{$2.1 \quad$ Teoria geral}

Antes de mais nada, vamos definir os quatro conceitos fundamentais deste capítulo.

Para todos os enunciados desta seção, sejam $\varphi: \mathcal{D} \rightarrow$ Card uma função cardinal, $\kappa$ um cardinal infinito e $\mathcal{S} \subseteq \mathcal{D}$. Note que todas as funções cardinais que consideraremos estão definidas para todo espaço topológico - logo, para tais funções, $\mathcal{D}=\mathcal{T}$ - com exceção do 
pseudocaráter, que é definida apenas em $\mathcal{T}_{1}$. Cabe lembrar que a propriedade $T_{1}$ é hereditária; assim, se $X \in \mathcal{T}_{1}$, temos que $\psi(Y)$ está definido para todo $Y \subseteq X$.

Definição 2.1.1. Dizemos que

(a) $\varphi$ reflete $\kappa$ para $\mathcal{S}$ se, e somente se, a seguinte afirmação é verdadeira para todo $X \in \mathcal{S}:$ "Se $\varphi(X) \geq \kappa$, então existe $Y \in[X] \leq \kappa$ tal que $\varphi(Y) \geq \kappa$." 1

(b) $\varphi$ reflete $\kappa$ fortemente para $\mathcal{S}$ se, e somente se, a seguinte afirmação é verdadeira para todo $X \in \mathcal{S}:$

"Se $\varphi(X) \geq \kappa$, então existe $Y \in[X]^{\leq \kappa}$ tal que $\varphi(Z) \geq \kappa$ para todo $Z \subseteq X$ verificando $Y \subseteq Z$."

(c) $\varphi$ tem a propriedade de Darboux em $\kappa$ para $\mathcal{S}$ se, e somente se, para todo $X \in \mathcal{S}$ tal que $\varphi(X)>\kappa$, existe $Y \subseteq X$ tal que $\varphi(Y)=\kappa$.

(d) $\varphi$ satisfaz $I U(\kappa)$ para $\mathcal{S}$ se, e somente se, a seguinte afirmação é verdadeira para todo $X \in \mathcal{S}:$

"Seja $\left(X_{\alpha}\right)_{\alpha \in \lambda}$ uma seqüência em $\mathcal{D}$ tal que

(i) $\lambda>\kappa$ é regular,

(ii) $\left(X_{\alpha}\right)_{\alpha \in \lambda}$ é crescente, ${ }^{2}$

(iii) $\varphi\left(X_{\alpha}\right)<\kappa$ para todo $\alpha \in \lambda e$

(iv) $X=\bigcup_{\alpha \in \lambda} X_{\alpha}$.

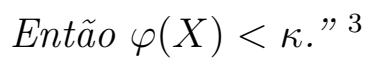

\footnotetext{
${ }^{1}$ Note que esta afirmação é equivalente a "se $\varphi(Y)<\kappa$ para todo $Y \in[X] \leq \kappa$, então $\varphi(X)<\kappa$ ".

${ }^{2}$ i.e., $\alpha<\beta \Rightarrow X_{\alpha} \subseteq X_{\beta}$ para quaisquer $\alpha, \beta \in \lambda$.

${ }^{3} \mathrm{~A}$ sigla $I U$ vem do inglês increasing union.
} 
Quando $\mathcal{S}$ não for especificado, assumiremos que $\mathcal{S}=\mathcal{D}$ - por exemplo, diremos apenas " $\varphi$ reflete $\kappa$ " para denotar que $\varphi$ reflete $\kappa$ para $\mathcal{D}$.

Note que, se $\kappa=\omega$, as propriedades $(a),(b)$ e $(d)$ são trivialmente satisfeitas por qualquer $\varphi$ para $\mathcal{D}$ - uma vez que, por definição, $\varphi(X) \geq \omega$ para qualquer $X \in \mathcal{D}$. Para as funções cardinais $\varphi$ que serão de nosso interesse nesta dissertação, o mesmo será válido para $(c)$, pois teremos que $\varphi(\{x\})=\omega$ sempre que $x \in X \in \mathcal{D}$.

Além disso, como os conceitos em 2.1.1 são definidos para $\kappa$ infinito, assumiremos que todos os cardinais considerados neste capítulo são infinitos. Assim, ao dizermos, por exemplo, que uma função cardinal $\varphi$ reflete todos os cardinais sucessores, entenda-se que $\varphi$ reflete todos os cardinais sucessores infinitos.

Vamos agora ver de que maneira tais conceitos estão relacionados entre si para uma mesma função cardinal.

Proposição 2.1.2. Valem as seguintes implicações:

(i) Se $\varphi$ reflete $\kappa$ fortemente para $\mathcal{S}$, então $\varphi$ reflete $\kappa$ para $\mathcal{S}$.

(ii) Se $\varphi$ é monótona e reflete $\kappa$ para $\mathcal{S}$, então $\varphi$ reflete $\kappa$ fortemente para $\mathcal{S}$.

(iii) Se $\varphi$ reflete $\kappa$ para $\mathcal{S}$ e $\varphi \leq|\cdot|$, então $\varphi$ tem a propriedade de Darboux em $\kappa$ para $\mathcal{S} .{ }^{4}$

(iv) Se $\varphi$ reflete $\kappa$ fortemente para $\mathcal{S}$, então $\varphi$ satisfaz $I U(\kappa)$ para $\mathcal{S}$.

Demonstração. Os itens (i) e (ii) decorrem imediatamente da definição 2.1.1.

Para (iii), suponha que $\varphi \leq|\cdot|$ e que $\varphi$ reflete $\kappa$ para $\mathcal{S}$. Se $X \in \mathcal{S}$ for tal que $\varphi(X)>\kappa$, então - como $\varphi$ reflete $\kappa$ para $\mathcal{S}-\varphi(Y) \geq \kappa$ para algum $Y \in[X]^{\leq \kappa}$. Assim, $\kappa \leq \varphi(Y) \leq$ $|Y|+\omega \leq \kappa, \log 0 \varphi(Y)=\kappa$.

\footnotetext{
${ }^{4}$ Por $\varphi \leq|\cdot|$, entenda-se que $\varphi(X) \leq|X|+\omega$ para todo espaço topológico $X$ no qual $\varphi$ está definida.
} 
Para (iv), assuma que $\varphi$ reflete $\kappa$ fortemente para $\mathcal{S}$ e sejam $X \in \mathcal{S}$ e $\left(X_{\alpha}\right)_{\alpha \in \lambda}$ uma seqüência crescente em $\mathcal{D}$ com $\lambda>\kappa$ regular, $\varphi\left(X_{\alpha}\right)<\kappa$ para todo $\alpha \in \lambda$ e $X=\bigcup_{\alpha \in \lambda} X_{\alpha}$. Suponha, por absurdo, que $\varphi(X) \geq \kappa$. Temos que $\varphi$ reflete $\kappa$ fortemente para $\mathcal{S}$, logo existe $Y \in[X]^{\leq \kappa}$ tal que $\varphi(Z) \geq \kappa$ para todo $Z \subseteq X \operatorname{com} Y \subseteq Z$. Como $Y \subseteq \bigcup_{\alpha \in \lambda} X_{\alpha},|Y| \leq \kappa<\lambda$, $\lambda$ é regular e $\left(X_{\alpha}\right)_{\alpha \in \lambda}$ é crescente, então existe $\alpha \in \lambda$ tal que $Y \subseteq X_{\alpha}$. Mas isto implica que $\varphi\left(X_{\alpha}\right) \geq \kappa$, uma contradição. Portanto, $\varphi(X)<\kappa$.

q.e.d.

Como já foi mencionado no início deste capítulo, nosso interesse se concentra nas propriedades de reflexão e reflexão forte de cardinais; assim, por exemplo, se a tese de um teorema deste capítulo for " $\varphi$ reflete $\kappa$ fortemente", julgaremos desnecessário salientar que $\varphi$ satisfaz $I U(\kappa)$.

O próximo lema será de grande valia no restante do capítulo.

Lema 2.1.3. Se $\varphi$ reflete fortemente todo cardinal sucessor para $\mathcal{S}$, então $\varphi$ reflete fortemente todo cardinal infinito para $\mathcal{S}$.

Demonstração. Assuma que $\varphi$ reflete fortemente todo cardinal sucessor para $\mathcal{S}$, e sejam $\kappa>\omega$ um cardinal limite e $X \in \mathcal{S}$ quaisquer. Suponha que $\varphi(X) \geq \kappa$. Para cada cardinal infinito $\lambda \in \kappa$, temos que $\varphi(X) \geq \lambda^{+}, \log 0-\operatorname{como} \varphi$ reflete $\lambda^{+}$fortemente para $\mathcal{S}$ - existe $Y_{\lambda} \subseteq X$ tal que $\left|Y_{\lambda}\right| \leq \lambda^{+}$e $\varphi(Z) \geq \lambda^{+}$para todo $Z \subseteq X$ tal que $Y_{\lambda} \subseteq Z$. Seja agora $Y=\bigcup\left\{Y_{\lambda} \mid \lambda \in\right.$ $\kappa, \lambda$ é cardinal infinito $\}$; temos então que $|Y| \leq \kappa$ e, se $Z \subseteq X$ é tal que $Y \subseteq Z$, segue que $Y_{\lambda} \subseteq Z$ para todo cardinal infinito $\lambda \in \kappa, \log \varphi(Z) \geq \lambda^{+}$para todo cardinal infinito $\lambda \in \kappa$, de modo que $\varphi(Z) \geq \kappa$.

q.e.d.

O lema seguinte nos permite obter propriedades de reflexão de $h \varphi$ a partir de propriedades de reflexão de $\varphi$.

Lema 2.1.4. Suponha que, para todo $X \in \mathcal{S}$, tenha-se $\wp(X) \subseteq \mathcal{S}$. Se $\varphi$ reflete $\kappa^{+}$para $\mathcal{S}$, então $h \varphi$ reflete $\kappa^{+}$fortemente para $\mathcal{S}$. 
Demonstração. Seja $X \in \mathcal{S}$ e suponha que $h \varphi(X) \geq \kappa^{+}$. Existe então $Y \subseteq X$ tal que $\varphi(Y) \geq \kappa^{+}$; assim, como $Y \in \mathcal{S}$ e $\varphi$ reflete $\kappa^{+}$para $\mathcal{S}$, segue que existe $Z \subseteq Y$ tal que $|Z| \leq \kappa^{+}$e $\varphi(Z) \geq \kappa^{+}$. Logo $h \varphi(Z) \geq \varphi(Z) \geq \kappa^{+}$e, portanto, $h \varphi$ reflete $\kappa^{+}$para $\mathcal{S}$. O resultado segue então da proposição 2.1.2(ii), pois $h \varphi$ é monótona.

q.e.d.

\subsection{Funções cardinais globais}

Nesta seção, trataremos de resultados de reflexão das funções cardinais $e, c, s, d, h d, L, h L$ e $w$. A referência principal é novamente [18]; referências bibliográficas adicionais serão ressaltadas no transcorrer do texto.

\subsubsection{Reflexão de $e, s$ e $c$}

Vamos começar pelas funções que apresentam as propriedades de reflexão mais gerais.

Teorema 2.2.1. As funções cardinais e, s e c refletem fortemente todos os cardinais infinitos.

Demonstração. Pelo lema 2.1.3, basta mostrar que tais funções cardinais refletem fortemente todos os cardinais sucessores. Fixe então um cardinal infinito $\kappa$ qualquer e tome $X \in \mathcal{T}$ arbitrário.

(i) Suponha que $e(X) \geq \kappa^{+}$; existe então $A \subseteq X$ discreto e fechado em $X$ tal que $|A| \geq \kappa^{+}$. Tome $Y \subseteq A \operatorname{com}|Y|=\kappa^{+}$. Como $A$ é discreto e fechado em $X$, então $A$ não admite nenhum ponto de acumulação; portanto, o mesmo ocorre com $Y$, o que implica que $Y$ é também discreto e fechado em $X$. Finalmente, note que, para todo $Z$ com $Y \subseteq Z \subseteq X$, tem-se que $Y$ é discreto e fechado em $Z$, de modo que $e(Z) \geq|Y|=\kappa^{+}$.

(ii) A demonstração para $s$ é análoga, pois todo subconjunto de um subespaço discreto de $X$ é também discreto. 
(iii) Suponha que $c(X) \geq \kappa^{+}$, e tome em $X$ uma família celular $\mathscr{C}$ com $|\mathscr{C}|=\kappa^{+}$. Para cada $U \in \mathscr{C}$, fixe $x_{U} \in U$, e considere $Y=\left\{x_{U} \mid U \in \mathscr{C}\right\}$ note que $|Y|=\kappa^{+}$. Tem-se, para todo $Z$ com $Y \subseteq Z \subseteq X$, que $\{U \cap Z \mid U \in \mathscr{C}\}$ é uma família celular em $Z$, $\operatorname{logo}$ $c(Z) \geq|\{U \cap Z \mid U \in \mathscr{C}\}|=|\mathscr{C}|=\kappa^{+}$.

q.e.d.

\subsubsection{Reflexão de $d$ e $h d$}

Passemos agora às funções cardinais $d$ e $h d$. No caso de $d$, os conceitos de reflexão e de propriedade de Darboux coincidem.

Proposição 2.2.2. Sejam $\kappa$ um cardinal infinito $e \mathcal{S} \subseteq \mathcal{T}$. Então d reflete $\kappa$ para $\mathcal{S}$ se, $e$ somente se, $d$ tem a propriedade de Darboux em $\kappa$ para $\mathcal{S}$.

Demonstração. Suponha que $d$ tem a propriedade de Darboux em $\kappa$ para $\mathcal{S}$. Seja $X \in \mathcal{S}$ tal que $d(X) \geq \kappa$; pela propriedade de Darboux, existe $Y \subseteq X$ tal que $d(Y)=\kappa$. Tome $D \subseteq Y$ denso em $Y \operatorname{com}|D|=\kappa$; temos então que $d(D)=\kappa$, logo $D \subseteq X$ testemunha a condição de reflexão em $X$.

A recíproca segue do item $($ iii) da proposição 2.1.2.

q.e.d.

A partir desta proposição, podemos agora enunciar como resultados de reflexão uma série de teoremas sobre a propriedade de Darboux demonstrados em [13] - a principal referência bibliográfica desta seção ${ }^{5}$.

O primeiro resultado de tal artigo que incluiremos é um lema auxiliar — do qual também faremos uso em outros pontos desta dissertação - :

\footnotetext{
${ }^{5}$ De modo geral, os resultados sobre $d$ aqui apresentados que se referem a reflexão provêm de [13], e os que se referem a reflexão forte provêm de [18].
} 
Proposição 2.2.3. Seja $(X, \tau)$ um espaço topológico tal que todo subespaço denso de $X$ é infinito. Então $(X, \tau)$ admite um subespaço denso $E \subseteq X$ que é separado à esquerda com tipo-de-ordem $d(X)$.

Demonstração. Tome $D \subseteq X$ denso em $X$ com $|D|=d(X)$, e fixe uma boa-ordem $\triangleleft$ em $D$ de modo que $t p(D)=d(X)$. Considere então

$$
E=\left\{\min _{\triangleleft}(U \cap D) \mid U \in \tau \backslash\{\emptyset\}\right\} .
$$

É imediato que $E$ é denso em $X$; assim, $|E|=d(X)$ e, portanto, $\triangleleft \uparrow(E \times E)$ é uma boa-ordem tal que $t p(E)=d(X)$.

Fixe agora $x \in E$ arbitrário. Temos que, para todo $y \in E \operatorname{com} x \unlhd y$, existe $U_{y} \in \tau$ tal que $y=\min _{\triangleleft}\left(U_{y} \cap D\right)$; para tal $U_{y}$, tem-se que $y=\min _{\triangleleft}\left(U_{y} \cap E\right)$, logo $y$ é ponto interior de $\{z \in E \mid y \unlhd z\} \subseteq\{z \in E \mid x \unlhd z\}$ na topologia de subespaço de $E$. Segue então que $\{z \in E \mid z \triangleleft x\} \subseteq E$ é fechado em $E$ e, como $x \in E$ foi tomado arbitrariamente, temos que $E$ é separado à esquerda.

q.e.d.

Podemos agora enunciar o primeiro teorema de reflexão sobre $d$, do qual decorre o fato de que $h d$ verifica todas as propriedades introduzidas em 2.1.1.

Teorema 2.2.4. A função cardinal d reflete todo cardinal regular.

Demonstração. Pela proposição 2.2.2, isto é equivalente a provar que $d$ tem a propriedade de Darboux em todo cardinal regular.

Seja então $\kappa$ um cardinal regular e suponha que $X \in \mathcal{T}$ é tal que $d(X)>\kappa$. Pela proposição 2.2.3, existe $E=\left\{x_{\alpha} \mid \alpha \in d(X)\right\} \subseteq X$ que é separado à esquerda e denso em $X$. Considere $F=\left\{x_{\alpha} \mid \alpha \in \kappa\right\} \subseteq E$.

Tome agora $A \in[F]^{<\kappa}$ arbitrário. Como $\kappa$ é regular e $|A|<\kappa$, então $\lambda=\sup \{\alpha \in$ $\left.\kappa \mid x_{\alpha} \in A\right\}+1 \in \kappa$; assim, $A \subseteq\left\{x_{\alpha} \mid \alpha \in \lambda\right\}$ e, como este é um conjunto fechado — já que $E$ é separado à esquerda -, segue que $\bar{A} \subseteq\left\{x_{\alpha} \mid \alpha \in \lambda\right\} \varsubsetneqq F, \operatorname{logo} A$ não é denso em $F$. Portanto, $d(F) \geq \kappa$ e, como $d(F) \leq|F|=\kappa$, temos que $d(F)=\kappa . \quad$ q.e.d. 
Corolário 2.2.5. A função cardinal hd reflete fortemente todos os cardinais infinitos.

Demonstração. Pelo teorema 2.2.4, d reflete todos os cardinais sucessores; logo, pelo lema 2.1.4, hd reflete fortemente todos os cardinais sucessores. O resultado segue então do lema 2.1.3.

q.e.d.

É natural agora perguntar sobre propriedades de reflexão de $d$ com respeito a cardinais singulares. O próximo resultado nos diz que nos restringir à classe dos espaços $T_{1}$ não é suficiente para a obtenção de uma resposta positiva.

Proposição 2.2.6. A função cardinal d não reflete nenhum cardinal singular para $\mathcal{T}_{1}$.

Demonstração. Seja $\lambda$ um cardinal singular. Pela proposição 2.2.2, isto é equivalente a provar que $d$ não possui a propriedade de Darboux em $\lambda$ para $\mathcal{T}_{1}$.

Considere, em $X=\lambda^{+}$, a topologia

$$
\tau=\left\{\left[\alpha, \lambda^{+}\left[\backslash F \mid \alpha \in \lambda^{+} \text {e } F \in\left[\lambda^{+}\right]^{<\omega}\right\}\right.\right.
$$

Note que $X$ é um espaço $T_{1}$ e que $d(X)=\lambda^{+}>\lambda$, uma vez que $\lambda^{+}$é um cardinal regular.

Fixe agora $Z \subseteq X$ arbitrário, e seja $\beta_{Z}$ o ordinal isomorfo em ordem a $Z$; existem então $k_{Z} \in \omega$ e um ordinal limite $\theta_{Z}$ tais que $\beta_{Z}=\theta_{Z}+k_{Z}$. Seja $R_{Z}$ o conjunto dos últimos $k_{Z}$ elementos de $Z$.

Afirmação: $D \subseteq Z$ é denso em $Z$ se, e somente se, existe $C$ cofinal em $Z \backslash R_{Z}$ tal que $D=C \cup R_{Z}$

De fato, seja $D \subseteq Z$ denso em $Z$. Segue da definição de $\tau$ que todo $\gamma \in R_{Z}$ é ponto isolado de $Z$, $\log$ o $R_{Z} \subseteq D$. Se $D \backslash R_{Z}$ não fosse cofinal em $Z \backslash R_{Z}$, teríamos que $\left(D \backslash R_{Z}\right) \cap\left[\delta, \lambda^{+}\left[=\emptyset\right.\right.$ para algum $\delta \in Z \backslash R_{Z}$; assim, $D \cap\left(\left[\delta, \lambda^{+}\left[\backslash R_{Z}\right)=\emptyset\right.\right.$, o que contradiria a hipótese de que $D$ é denso em $Z$. 
Reciprocamente, seja $C$ cofinal em $Z \backslash R_{Z}$. Para $\alpha \in \sup \left(Z \backslash R_{Z}\right)$ e $F \in\left[\lambda^{+}\right]^{<\omega}$ quaisquer, temos que $\left(\left[\alpha, \lambda^{+}[\backslash F) \cap\left(C \cup R_{Z}\right) \neq \emptyset\right.\right.$ - pois, como $C \subseteq Z \backslash R_{Z}$ é cofinal, então $\left[\alpha, \lambda^{+}\left[\cap C\right.\right.$ é infinito. Já se $\alpha \geq \sup \left(Z \backslash R_{Z}\right)$, é claro que, para todo $F \in\left[\lambda^{+}\right]^{<\omega}$, tem-se que $\left(\left[\alpha, \lambda^{+}[\backslash F) \cap\left(C \cup R_{Z}\right)=\emptyset\right.\right.$ implica $\left(\left[\alpha, \lambda^{+}[\backslash F) \cap Z=\emptyset\right.\right.$. Portanto, $C \cup R_{Z}$ é denso em $Z$.

Assim, $d(Z)=\min \left\{|C| \mid C\right.$ é cofinal em $\left.Z \backslash R_{Z}\right\}=c f\left(\theta_{Z}\right)$, o que implica que $d(Z)$ é regular e, portanto, $d(Z) \neq \lambda$.

q.e.d.

A situação muda, no entanto, ao considerarmos a classe dos espaços de Hausdorff.

Teorema 2.2.7. A função cardinal d reflete fortemente todo cardinal limite forte para $\mathcal{T}_{2}$.

Demonstração. Seja $\kappa$ um cardinal limite forte. Se $X \in \mathcal{T}_{2}$ é tal que $d(X) \geq \kappa$, temos que $|X| \geq \kappa$; fixe então $Y \subseteq X$ com $|Y|=\kappa$. Se existisse $Z \subseteq X$ com $Y \subseteq Z$ e $d(Z)<\kappa$, teríamos pela desigualdade ${ }^{6}|Z| \leq 2^{2^{d(Z)}}$ que $|Z|<\kappa$, uma contradição. q.e.d.

Corolário 2.2.8. Assumindo-se GCH, d reflete todos os cardinais infinitos para $\mathcal{T}_{2}$.

Demonstração. GCH implica que todo cardinal limite é limite forte, logo todo cardinal singular é limite forte e o resultado decorre dos resultados 2.2.4, 2.2.7 e 2.1.2(i).

q.e.d.

Antes do próximo teorema, que trata o caso particular em que $c f(\lambda)=\omega$, mostremos o seguinte lema.

Lema 2.2.9. Todo espaço de Hausdorff infinito possui uma família celular infinita.

\footnotetext{
${ }^{6}$ Este resultado é devido a B. Pospíšil, e sua demonstração é simples: basta tomar $D \subseteq Z$ denso em $Z$ com $|D| \leq d(Z)$ e notar que, como $Z$ verifica $T_{2}$, então a função

$$
\begin{aligned}
f: Z & \rightarrow \wp(\wp(D)) \\
z & \mapsto\{A \subseteq D \mid z \in \bar{A}\}
\end{aligned}
$$

é injetora.
} 
Demonstração. Seja $(X, \tau)$ um espaço de Hausdorff tal que $X$ é infinito, e considere $E=\{x \in$ $X \mid x$ é ponto isolado em $(X, \tau)\}$. Se $E$ for infinito, basta tomar $\{\{x\} \mid x \in E\} \subseteq \tau$. Suponha então que $E$ seja finito - note que, neste caso, $E$ é fechado em $(X, \tau)$. Vamos construir, indutivamente, seqüências $\left(U_{n}\right)_{n \in \omega}$ e $\left(V_{n}\right)_{n \in \omega}$ em $\tau$ e $\left(x_{n}\right)_{n \in \omega}$ em $X \backslash E$ como segue.

Defina $V_{0}=X \backslash E$ e tome $x_{0} \in V_{0}$ arbitrário. Suponha agora que, para um certo $k \in \omega$, já tenhamos construído as seqüências $\left(U_{n}\right)_{n \in k},\left(V_{n}\right)_{n \in(k+1)}$ e $\left(x_{n}\right)_{n \in(k+1)}$. Tome $x_{k+1} \in V_{k} \backslash\left\{x_{k}\right\}$ arbitrário - note que $V_{k} \backslash\left\{x_{k}\right\} \neq \emptyset$, pois $x_{k} \notin E$. Como $(X, \tau)$ é um espaço de Hausdorff, existem $\Omega, \Omega^{\prime} \in \tau$ disjuntos tais que $x_{k} \in \Omega$ e $x_{k+1} \in \Omega^{\prime}$; defina então $U_{k}=\Omega \cap V_{k}$ e $V_{k+1}=\Omega^{\prime} \cap V_{k}$.

Note que, por construção, $x_{n} \in U_{n} \subseteq V_{n} \subseteq V_{0}=X \backslash E$ para todo $n \in \omega-$ pois $V_{n+1} \subseteq V_{n}$ para todo $n \in \omega-$, de modo que, de fato, $\left\{x_{n} \mid n \in \omega\right\} \subseteq X \backslash E$.

Provemos agora, por indução, que $V_{n} \cap \bigcup\left\{U_{m} \mid m \in n\right\}=\emptyset$ para todo $n \in \omega$. O caso $n=0$ é trivial. Suponha agora que a afirmação seja válida para um certo $k \in \omega$. Como já observamos, $V_{k+1} \subseteq V_{k}, \log V_{k+1} \cap \bigcup\left\{U_{m} \mid m \in k\right\}=\emptyset$. Já $V_{k+1} \cap U_{k}=\emptyset$ decorre diretamente da construção e, assim, a afirmação é verdadeira para $k+1$.

Segue então que $U_{n} \cap \bigcup\left\{U_{m} \mid m \in n\right\} \subseteq V_{n} \cap \bigcup\left\{U_{m} \mid m \in n\right\}=\emptyset$ para todo $n \in \omega \mathrm{e}$, portanto, $\left\{U_{n} \mid n \in \omega\right\} \subseteq \tau$ é uma família nas condições desejadas. q.e.d.

Teorema 2.2.10. Seja $\lambda$ um cardinal singular tal que $\operatorname{cf}(\lambda)=\omega$. Então $d$ reflete $\lambda$ para $\mathcal{T}_{2}$.

Demonstração. Provaremos que $d$ tem a propriedade de Darboux em $\lambda$ para $\mathcal{T}_{2}$, e o resultado seguirá então da proposição 2.2.2.

Tome $X \in \mathcal{T}_{2}$ tal que $d(X)>\lambda$. Pela proposição 2.2.3, existe $E \subseteq X$ denso em $X$ e separado à esquerda com uma boa-ordem $\triangleleft$ tal que $t p(E)=d(X)$; seja então $S \subseteq E$ o segmento inicial de $E$ de comprimento $\lambda^{+}$, e considere $\mathcal{W}=\{W \subseteq S \mid W$ é aberto em $S$ e $|W| \leq \lambda\}$. Temos dois casos a considerar:

(i) $|\bigcup \mathcal{W}|=\lambda^{+}$. 
Construa seqüências $\left(x_{\alpha}\right)_{\alpha \in \lambda}$ em $\bigcup \mathcal{W}$ e $\left(W_{\alpha}\right)_{\alpha \in \lambda}$ em $\mathcal{W}$ como segue.

Suponha que, para um certo $\beta \in \lambda$, já tenhamos construído $\left(x_{\alpha}\right)_{\alpha \in \beta}$ e $\left(W_{\alpha}\right)_{\alpha \in \beta}$. Temos que $\left|\bigcup_{\alpha \in \beta} W_{\alpha}\right| \leq \lambda \cdot|\beta| \leq \lambda \cdot \lambda=\lambda$, $\log \mathrm{o}(\bigcup \mathcal{W}) \backslash\left(\bigcup_{\alpha \in \beta} W_{\alpha}\right) \neq \emptyset$; seja então

$$
x_{\beta}=\min _{\triangleleft}\left((\bigcup \mathcal{W}) \backslash\left(\bigcup_{\alpha \in \beta} W_{\alpha}\right)\right)
$$

e tome $W_{\beta} \in \mathcal{W}$ arbitrário $\operatorname{com} x_{\beta} \in W_{\beta}$.

Provemos agora que $E=\left\{x_{\alpha} \mid \alpha \in \lambda\right\}$ é discreto — e, portanto, $d(E)=|E|=\lambda$, como procurávamos.

Para cada $\alpha \in \lambda$, tome $U_{\alpha} \subseteq S$ aberto em $S$ tal que $x_{\alpha}=\min _{\triangleleft} U_{\alpha}$ - um tal $U_{\alpha}$ existe, pois $S$ é separado à esquerda. Fixe agora $\beta \in \lambda$ e suponha que $\alpha \in \lambda$ seja tal que $x_{\alpha} \in U_{\beta} \cap W_{\beta} \cap E$. De $x_{\alpha} \in U_{\beta} \cap E$ segue que $\alpha \geq \beta$, e de $x_{\alpha} \in W_{\beta} \cap E$ segue que $\alpha \leq \beta$; assim, $U_{\beta} \cap W_{\beta} \cap E=\left\{x_{\beta}\right\}$, logo $x_{\beta}$ é um ponto isolado de $E$.

(ii) $|\bigcup \mathcal{W}| \leq \lambda$

Seja $Z=S \backslash \bigcup \mathcal{W}$, e tome $\left\{\kappa_{n} \mid n \in \omega\right\} \subseteq \lambda$ tal que $\sup \left\{\kappa_{n} \mid n \in \omega\right\}=\lambda$; podemos supor que $\kappa_{n}$ é regular para todo $n \in \omega$, tomando, se necessário, $\kappa_{n}^{+}$ao invés de $\kappa_{n}$. Pelo lema anterior, existe uma família infinita $\left\{U_{n} \mid n \in \omega\right\}$ de abertos de $Z$ não-vazios e dois a dois disjuntos; note que $\left|U_{n}\right|=\lambda^{+}$para todo $n \in \omega$, pois, se $\Omega \subseteq S$ é um aberto de $S$ tal que $|\Omega \cap Z| \leq \lambda$, então $|\Omega|=|\Omega \cap Z|+|\Omega \cap \bigcup \mathcal{W}| \leq \lambda+\lambda=\lambda$, logo $\Omega \in \mathcal{W}$ e, portanto, $\Omega \cap Z=\emptyset$.

Fixe agora $n \in \omega$ arbitrário. Como $Z$ é separado à esquerda com $t p(Z)=\lambda^{+}$, o mesmo ocorre com $U_{n}$; logo, sendo $E_{n} \subseteq U_{n}$ o segmento inicial de $U_{n}$ de comprimento $\kappa_{n}$, temos que $d\left(E_{n}\right)=\left|E_{n}\right|=\kappa_{n}$, pois $\kappa_{n}$ é regular. $^{7}$

Seja então $E=\bigcup_{n \in \omega} E_{n} \subseteq Z$; provaremos que $d(E)=\lambda$, o que concluirá a demonstração.

\footnotetext{
${ }^{7}$ Análogo à demonstração do teorema 2.2.4.
} 
Por um lado, $d(E) \leq \lambda$ segue de

$$
|E|=\left|\bigcup_{n \in \omega} E_{n}\right|=\sum_{n \in \omega}\left|E_{n}\right|=\sum_{n \in \omega} \kappa_{n}=\lambda .
$$

Para a outra desigualdade, tome $D \subseteq E$ denso em $E$ arbitrário; para cada $n \in \omega$, temos que $E_{n}$ é aberto em $E$, pois $E_{n}=U_{n} \cap E$ e $U_{n} \subseteq Z$ é aberto em $Z$. Assim sendo, $D \cap E_{n}$ é denso em $E_{n}$ para todo $n \in \omega, \operatorname{logo}\left|D \cap E_{n}\right| \geq d\left(E_{n}\right)=\kappa_{n}$ e, portanto,

$$
|D|=\sum_{n \in \omega}\left|D \cap E_{n}\right| \geq \sum_{n \in \omega} \kappa_{n}=\lambda
$$

de modo que $d(E) \geq \lambda$.

q.e.d.

Tratemos agora da reflexão forte de cardinais por $d$ - para a qual, excetuando-se o teorema 2.2.7, há apenas resultados negativos, como os que se seguem.

Proposição 2.2.11. Seja $\kappa$ um cardinal não-enumerável. Então a função cardinal d não satisfaz $I U(\kappa)-e$, portanto ${ }^{8}$, não reflete $\kappa$ fortemente - para $\mathcal{T}_{1}$.

Demonstração. O espaço que nos servirá de contra-exemplo é o mesmo utilizado na demonstração da proposição 2.2.6: consideramos, em $X=\kappa^{+}$, a topologia

$$
\tau=\left\{\left[\alpha, \kappa^{+}\left[\backslash F \mid \alpha \in \kappa^{+} \text {e } F \in\left[\kappa^{+}\right]^{<\omega}\right\}\right.\right.
$$

que o torna um espaço $T_{1}$ de densidade $\kappa^{+}$.

Construamos, por indução transfinita, uma seqüência crescente $\left(X_{\alpha}\right)_{\alpha \in \kappa^{+}}$de subconjuntos de $X$ tal que, para todo $\alpha \in \kappa^{+}$,

(i) $\alpha \in X_{\alpha}$,

\footnotetext{
${ }^{8}$ Em virtude de 2.1.2(iv).
} 
(ii) $\left|X_{\alpha}\right| \leq \kappa$,

(iii) $d\left(X_{\alpha}\right)=\omega$

Suponha que, para $\beta \in \kappa^{+}$, já tenhamos construído $\left(X_{\alpha}\right)_{\alpha \in \beta}$ satisfazendo as condições $(i)$, (ii) e (iii) para todo $\alpha \in \beta$. Seja $\gamma=\sup \left(\{\beta\} \cup \bigcup_{\alpha \in \beta} X_{\alpha}\right)$; como $|\beta| \leq \kappa$ e $\left|X_{\alpha}\right| \leq \kappa$ para todo $\alpha \in \beta$, segue da regularidade de $\kappa^{+}$que $\gamma \in \kappa^{+}$. Tome então uma seqüência estritamente crescente $\left(\alpha_{n}\right)_{n \in \omega}$ em $\kappa^{+}$tal que $\alpha_{0}=\gamma$, e defina

$$
X_{\beta}=\{\beta\} \cup\left(\bigcup_{\alpha \in \beta} X_{\alpha}\right) \cup\left\{\alpha_{n} \mid n \in \omega\right\} .
$$

Desta forma, temos que $X_{\beta}$ verifica as condições $(i)$ e $(i i)$, e a condição $(i i i)$ decorre do fato de que $\left\{\alpha_{n} \mid n \in \omega\right\}$ é denso em $X_{\beta}$.

Concluímos então a construção de $\left(X_{\alpha}\right)_{\alpha \in \kappa^{+}}$e, assim, a demonstração — uma vez que $d(X)=\kappa^{+}$e a condição $(i)$ implica que $X=\bigcup_{\alpha \in \kappa^{+}} X_{\alpha}$.

q.e.d.

Proposição 2.2.12. Seja $\kappa$ um cardinal infinito. Então a função cardinal d não verifica $I U\left(\kappa^{+}\right)$- e, portanto, não reflete $\kappa^{+}$fortemente - para a classe dos espaços completamente regulares.

Demonstração. Considere o espaço ${ }^{\kappa^{++}}\{0,1\}$ com a topologia produto e defina, para cada $\alpha \in \kappa^{++}$,

$$
X_{\alpha}=\left\{f \in \kappa ^ { \kappa ^ { + + } } \{ 0 , 1 \} | f ( \beta ) = 0 \text { para todo } \beta \in \left[\alpha, \kappa^{++}[\} .\right.\right.
$$

Fixe $\alpha \in \kappa^{++}$arbitrário. Pelo teorema de Hewitt-Marczewski-Pondiczery ${ }^{9}$, temos que $d\left(X_{\alpha}\right) \leq \kappa<\kappa^{+}$, pois $X_{\alpha}$ é homeomorfo a $\{0,1\}^{\alpha}$ e $|\alpha| \leq \kappa^{+} \leq 2^{\kappa}$.

Afirmamos agora que $X=\bigcup_{\alpha \in \kappa^{++}} X_{\alpha} \subseteq \kappa^{++}\{0,1\}$ tem densidade maior ou igual a $\kappa^{++}$, o que concluirá a demonstração — note que $X$ é um espaço completamente regular, pois $\kappa^{++}\{0,1\}$ o é. De fato, dado $D \in[X]^{\leq \kappa^{+}}$, tem-se pela regularidade de $\kappa^{++}$que existe $\alpha \in \kappa^{++}$

\footnotetext{
${ }^{9}$ Vide seção 1.2.7.
} 
tal que $D \subseteq X_{\alpha}$. Como $\{f \in X \mid f(\alpha)=1\}$ é um aberto de $X$ disjunto de $X_{\alpha}$, então $X \neq \overline{X_{\alpha}} \supseteq \bar{D}$, e disto segue que $D$ não é denso em $X$.

q.e.d.

\subsubsection{Reflexão de $L$ e $h L$}

Tratemos agora de $L$ e $h L$. Assim como foi visto nos resultados que dizem respeito a $d$ e $h d$, o primeiro resultado sobre $L$ terá como conseqüência que $h L$ reflete fortemente todos os cardinais infinitos.

Teorema 2.2.13. A função cardinal L reflete todo cardinal sucessor.

Demonstração. Sejam $\kappa$ um cardinal infinito e $X \in \mathcal{T}$ tais que $L(X) \geq \kappa^{+}$. Segue então da definição de $L$ que existe um recobrimento aberto $\mathscr{U}$ de $X$ que não admite sub-recobrimento de cardinalidade menor ou igual a $\kappa$.

Construa, por indução transfinita, $\left\{U_{\alpha} \mid \alpha \in \kappa^{+}\right\} \subseteq \mathscr{U}$ e $Y=\left\{x_{\alpha} \mid \alpha \in \kappa^{+}\right\} \subseteq X$ tais que, para todo $\alpha \in \kappa^{+}$,

$$
x_{\alpha} \in U_{\alpha} \backslash \bigcup_{\beta \in \alpha} U_{\beta} .
$$

Para tanto, tendo $\left\{U_{\alpha} \mid \alpha \in \gamma\right\}$ e $\left\{x_{\alpha} \mid \alpha \in \gamma\right\}$ já construídos para $\gamma \in \kappa^{+}$, basta escolher $x_{\gamma} \in X \backslash \bigcup_{\alpha \in \gamma} U_{\alpha}$ - que é não-vazio, pela hipótese sobre $\mathscr{U}-$ e $U_{\gamma} \in \mathscr{U} \operatorname{com} x_{\gamma} \in U_{\gamma}$.

Assim, $Y \subseteq X$ é tal que $|Y|=\kappa^{+}$e $L(Y) \geq \kappa^{+}$- pois $\left\{U_{\alpha} \cap Y \mid \alpha \in \kappa^{+}\right\}$é um recobrimento aberto de $Y$ que não admite sub-recobrimento de cardinalidade menor ou igual a $\kappa$.

Corolário 2.2.14. A função cardinal hL reflete fortemente todos os cardinais infinitos.

Demonstração. Segue diretamente do teorema anterior e dos lemas 2.1.4 e 2.1.3. q.e.d.

No que diz respeito aos cardinais singulares, a situação novamente é análoga à que ocorre com $d$. 
Proposição 2.2.15. Seja $\lambda$ um cardinal singular. Então L não possui a propriedade de Darboux em $\lambda-e$, portanto $^{10}$, não reflete $\lambda$ - para $\mathcal{T}_{1}$.

Demonstração. O exemplo que construiremos é, num certo sentido, o dual do exemplo construído na demonstração da proposição 2.2.6. ${ }^{11}$

Considere, em $X=\lambda^{+}$, a topologia

$$
\tau=\left\{\left[0, \alpha\left[\backslash F \mid \alpha \in \lambda^{+} \text {e } F \in\left[\lambda^{+}\right]^{<\omega}\right\} .\right.\right.
$$

Note que $X$ é um espaço $T_{1}$ e que $L(X)=\lambda^{+}>\lambda$ - para tanto, considere o recobrimento $\left\{\left[0, \alpha\left[\mid \alpha \in \lambda^{+}\right\}\right.\right.$.

Tome $Y \subseteq X$ arbitrário; vamos mostrar que $L(Y) \neq \lambda$.

Se $|Y|=\lambda^{+}$, então $Y$ é cofinal em $\lambda^{+}, \operatorname{logo} t p(Y)=\lambda^{+}$e, por um argumento análogo ao do caso $Y=X$, segue que $L(Y)=\lambda^{+}$. Suponha então $|Y|<\lambda^{+}$, e seja $\rho \in \lambda^{+}$o ordinal isomorfo à ordem de $Y$. Temos dois casos a considerar:

(i) $\rho$ é um ordinal sucessor. Afirmamos que então $Y$ é compacto - e, portanto, $L(Y)=$ $\omega \neq \lambda$.

De fato, seja $\mathscr{C}$ um recobrimento aberto qualquer de $Y$. Como o tipo-de-ordem de $Y$ é um ordinal sucessor, então $Y$ possui elemento máximo; seja $\eta$ tal elemento. Tome $V \in \mathscr{C}$ com $\eta \in V$; pela definição de $\tau$, o conjunto $Y \backslash V$ é finito. Basta então tomar, para cada $\xi \in Y \backslash V$, um aberto $U_{\xi} \in \mathscr{C}$ tal que $\xi \in U_{\xi}$, e $\{V\} \cup\left\{U_{\xi} \mid \xi \in Y \backslash V\right\}$ será um sub-recobrimento finito de $\mathscr{C}$.

(ii) $\rho$ é um ordinal limite. Neste caso, temos que $L(Y)=c f(\rho) \neq \lambda$ - pois $c f(\rho)$ é um cardinal regular.

\footnotetext{
${ }^{10}$ Por 2.1.2(iii).

${ }^{11}$ De modo geral, exemplos interessantes para resultados sobre $d$ e $L$ podem ser obtidos ao se considerar, respectivamente, espaços separados à esquerda e espaços separados à direita.
} 
Para tanto, notemos, em primeiro lugar, que $L(Y) \geq c f(\rho)$, pois o recobrimento

$$
\{Y \cap[0, \alpha] \mid \alpha \in Y\}
$$

não admite sub-recobrimento de cardinalidade menor que $c f(\rho)$ - pela definição de cofinalidade.

Seja agora $\mathscr{C}$ um recobrimento aberto arbitrário de $Y$. Para cada $\alpha \in Y$, fixe $U_{\alpha} \in \mathscr{C}$ com $\alpha \in U_{\alpha}$. Tome agora $Z \subseteq Y$ cofinal em $Y \operatorname{com}|Z|=c f(\rho)$ - tal $Z$ existe, pois $Y$ e $\rho$ são isomorfos em ordem. Pela definição de $\tau$, para cada $\alpha \in Z$ tem-se que $D_{\alpha}=$ $(Y \cap[0, \alpha]) \backslash U_{\alpha}$ é finito; defina, então, $\mathscr{A}_{\alpha}=\left\{U_{\alpha}\right\} \cup\left\{U_{\beta} \mid \beta \in D_{\alpha}\right\}$ para todo $\alpha \in Z$. Note que $Y \cap[0, \alpha] \subseteq \bigcup \mathscr{A}_{\alpha}$ para todo $\alpha \in Z$, de modo que $\mathscr{C}^{\prime}=\bigcup\left\{\mathscr{A}_{\alpha} \mid \alpha \in Z\right\} \subseteq \mathscr{C}$ é um recobrimento de $Y$ que satisfaz $\left|\mathscr{C}^{\prime}\right|=|Z|=c f(\rho)$. Provamos então que $L(Y) \leq c f(\rho)$, como desejado.

Portanto, não existe $Y \subseteq X$ tal que $L(Y)=\lambda$, de modo que $L$ não possui a propriedade de Darboux em $\lambda$ para $\mathcal{T}_{1}$.

q.e.d.

Os resultados obtidos para $L$ na classe dos espaços de Hausdorff também apresentam semelhanças com os de $d$. Para esse caso, será importante o seguinte teorema, demonstrado em [14]:

Teorema 2.2.16. Seja $(X, \tau) \in \mathcal{T}_{2}$ tal que $\lambda=|X|$ é um cardinal limite forte e singular. Então existe $D \subseteq X$ discreto com $|D|=\lambda$.

Demonstração. Fixe uma boa-ordem $\triangleleft$ sobre $X$ e, para cada par $(x, y) \in X \times X$ tal que $x \triangleleft y$, tome $U_{(x, y)}, V_{(x, y)} \in \tau$ disjuntos tais que $x \in U_{(x, y)}$ e $y \in V_{(x, y)}$. Considere, agora, $\varphi_{1}:[X]^{3} \rightarrow\{0,1,2\}$ e $\varphi_{2}:[X]^{3} \rightarrow\{0,1,2\}$ tais que, para todo $\{x, y, z\} \in[X]^{3} \operatorname{com} x \triangleleft y \triangleleft z$,

- $\varphi_{1}(\{x, y, z\})=0$ se, e somente se, $x \notin U_{(y, z)} \cup V_{(y, z)}$;

- $\varphi_{1}(\{x, y, z\})=1$ se, e somente se, $x \in U_{(y, z)}$; 
- $\varphi_{1}(\{x, y, z\})=2$ se, e somente se, $x \in V_{(y, z)}$

- $\varphi_{2}(\{x, y, z\})=0$ se, e somente se, $z \notin U_{(x, y)} \cup V_{(x, y)}$;

- $\varphi_{2}(\{x, y, z\})=1$ se, e somente se, $z \in U_{(x, y)}$;

- $\varphi_{2}(\{x, y, z\})=2$ se, e somente se, $z \in V_{(x, y)}$.

Vamos agora definir uma partição $\left\{\mathcal{P}_{\left(a_{1}, a_{2}\right)} \mid\left(a_{1}, a_{2}\right) \in\{0,1,2\} \times\{0,1,2\}\right\}$ de $[X]^{3}$ por

$$
\{x, y, z\} \in \mathcal{P}_{\left(a_{1}, a_{2}\right)} \Leftrightarrow\left(\varphi_{1}(\{x, y, z\})=a_{1} \text { e } \varphi_{2}(\{x, y, z\})=a_{2}\right)
$$

para todo $\{x, y, z\} \in[X]^{3}$ tal que $x \triangleleft y \triangleleft z$ e todo $\left(a_{1}, a_{2}\right) \in\{0,1,2\} \times\{0,1,2\}$.

Aplicando agora o lema da canonização ${ }^{12}$, obtemos $S \subseteq X$ e uma partição $\left\{S_{\xi} \mid \xi \in \sigma\right\}$ de $S$ - sendo $\sigma=c f(\lambda)$ - satisfazendo

(i) $\left(\left|S_{\xi}\right|\right)_{\xi \in \sigma}$ é uma seqüência estritamente crescente de cardinais infinitos e tem limite $\lambda$.

(ii) Se $\xi_{1}, \xi_{2} \in \sigma$ são tais que $\xi_{1}<\xi_{2}$, tem-se que $x_{1} \triangleleft x_{2}$ para quaisquer $x_{1} \in S_{\xi_{1}}$ e $x_{2} \in S_{\xi_{2}}$.

(iii) Se $A, B \in[S]^{3}$ são tais que $\left|A \cap S_{\xi}\right|=\left|B \cap S_{\xi}\right|$ para todo $\xi \in \sigma$, então $\varphi_{1}(A)=\varphi_{1}(B)$ e $\varphi_{2}(A)=\varphi_{2}(B)$.

Podemos assumir que cada $S_{\xi}$ tem tipo-de-ordem $\left|S_{\xi}\right|$ com respeito a $\triangleleft$, pois a partição $\left\{S_{\xi}^{\prime} \mid \xi \in \sigma\right\}$ obtida tomando-se os segmentos iniciais de comprimento $\left|S_{\xi}\right|$ com respeito a $\triangleleft$ em cada $S_{\xi}$ também verifica as condições $(i),($ ii $)$ e $(i i i)$ do lema. Para cada $x \in S$, sejam $x^{+}$ o sucessor de $x$ e $x^{++}$o sucessor de $x^{+}$com respeito a $\triangleleft$.

Afirmamos que, para todo $x \in S$,

$$
S \cap V_{\left(x, x^{+}\right)} \cap U_{\left(x^{+}, x^{++}\right)}=\left\{x^{+}\right\},
$$

\footnotetext{
${ }^{12}$ Vide apêndice $\mathrm{A}$.
} 
$\operatorname{logo} x^{+}$é um ponto isolado de $S$. Isto concluirá a demonstração, pois implicará que $S^{+}=$ $\left\{x^{+} \mid x \in S\right\}$ é um subespaço discreto de $X$ e satisfaz $\left|S^{+}\right|=|S|=\lambda$.

De fato, tome $x \in S$ arbitrário e seja $\xi_{x} \in \sigma$ tal que $x \in S_{\xi_{x}}$; note que, da condição (ii) e da suposição de que $t p\left(S_{\xi_{x}}\right)=\left|S_{\xi_{x}}\right|$, segue que $x^{+}, x^{++} \in S_{\xi_{x}}$. Agora suponha, por absurdo, que exista

$$
y \in S \cap V_{\left(x, x^{+}\right)} \cap U_{\left(x^{+}, x^{++}\right)} \backslash\left\{x^{+}\right\} .
$$

Pela definição de $U_{\left(x^{+}, x^{++}\right)}$e de $V_{\left(x, x^{+}\right)}$, temos que $y \neq x$ e $y \neq x^{++}$. Há então dois casos a se considerar:

- Se $y \triangleleft x$, segue da condição (iii) que $\varphi_{1}\left(\left\{y, x, x^{+}\right\}\right)=\varphi_{1}\left(\left\{y, x^{+}, x^{++}\right\}\right)$. Mas isto é uma contradição, pois $y \in V_{\left(x, x^{+}\right)}$implica que $\varphi_{1}\left(\left\{y, x, x^{+}\right\}\right)=2$ e $y \in U_{\left(x^{+}, x^{++}\right)}$implica que $\varphi_{1}\left(\left\{y, x^{+}, x^{++}\right\}\right)=1$.

- Se $x^{++} \triangleleft y$, segue igualmente da condição (iii) que $\varphi_{2}\left(\left\{x, x^{+}, y\right\}\right)=\varphi_{2}\left(\left\{x^{+}, x^{++}, y\right\}\right)$. Temos novamente um absurdo, uma vez que $y \in V_{\left(x, x^{+}\right)}$implica que $\varphi_{2}\left(\left\{x, x^{+}, y\right\}\right)=2$ e $y \in U_{\left(x^{+}, x^{++}\right)}$implica que $\varphi_{2}\left(\left\{x^{+}, x^{++}, y\right\}\right)=1$.

q.e.d.

Corolário 2.2.17. Seja $\lambda$ um cardinal limite forte e singular. Então $L$ reflete $\lambda$ para $\mathcal{T}_{2}$.

Demonstração. Tome $X \in \mathcal{T}_{2}$ e suponha que $L(X) \geq \lambda$. Então $|X| \geq \lambda$, $\operatorname{logo}$ existe $Y \subseteq X$ com $|Y|=\lambda$. Pelo teorema 2.2.16, existe $D \subseteq Y$ discreto com $|D|=\lambda$. Por ser discreto, tal $D$ satisfaz $L(D)=\lambda$, testemunhando a reflexão.

q.e.d.

Corolário 2.2.18. Assuma GCH e a não-existência de cardinais fortemente inacessiveis ${ }^{13}$. Então $L$ reflete todo cardinal infinito para $\mathcal{T}_{2}$.

Demonstração. Seja $\kappa$ um cardinal infinito. Se $\kappa$ é sucessor, a afirmação decorre do teorema 2.2.13. Se $\kappa$ é limite, segue de GCH que $\kappa$ é limite forte; temos ainda que $\kappa$ é singular -

\footnotetext{
${ }^{13}$ Esta hipótese é consistente com ZFC; vide, e.g., [25], capítulo VI, corolário 4.13.
} 
pois, do contrário, $\kappa$ seria fortemente inacessível - , e o resultado segue então do corolário anterior.

A última proposição que apresentamos sobre $L$ diz respeito à reflexão forte de cardinais para a qual não se conhecem resultados positivos.

Proposição 2.2.19. Seja $\kappa$ um cardinal não-enumerável. Então L não satisfaz $I U(\kappa)-e$, portanto, não reflete $\kappa$ fortemente - para a classe dos espaços hereditariamente normais.

Demonstração. Tome $X=\kappa^{+}$com a topologia da ordem — que é um espaço hereditariamente normal. Note que $L(X)=\kappa^{+}$, já que $\kappa^{+}$é regular e, portanto, o recobrimento $\left\{\left[0, \alpha\left[\mid \alpha \in \kappa^{+}\right\}\right.\right.$ não admite sub-recobrimento de cardinalidade menor que $\kappa^{+}$.

Defina, para todo $\alpha \in \kappa^{+}, X_{\alpha}=[0, \alpha]$. Então todo $X_{\alpha}$ é compacto, $\operatorname{logo} L\left(X_{\alpha}\right)=\omega$ para todo $\alpha \in \kappa^{+}$. Como $X=\bigcup_{\alpha \in \kappa^{+}} X_{\alpha}$, segue então que $L$ não verifica $I U(\kappa)$. q.e.d.

\subsubsection{Reflexão de $w$}

Finalmente, demonstraremos o teorema de A. Hajnal e I. Juhász — [16] — sobre a reflexão do peso:

Teorema 2.2.20. A função cardinal $w$ reflete fortemente todos os cardinais infinitos.

Mas, antes, precisaremos do seguinte lema:

Lema 2.2.21. Seja $\mathcal{B}$ uma base de abertos para um espaço topológico $(X, \tau)$. Então existe $\mathcal{B}^{\prime} \subseteq \mathcal{B}$ tal que $\mathcal{B}^{\prime}$ é base para $X$ e $\left|\mathcal{B}^{\prime}\right| \leq w(X)$.

Demonstração. Fixe uma base de abertos $\mathcal{U}$ para $X$ tal que $|\mathcal{U}| \leq w(X)$. Considere

$$
\mathcal{P}=\left\{\left(U_{1}, U_{2}\right) \in \mathcal{U} \times \mathcal{U} \mid \exists W \in \mathcal{B}\left(U_{1} \subseteq W \subseteq U_{2}\right)\right\},
$$

e tome $\varphi: \mathcal{P} \rightarrow \mathcal{B}$ tal que $U_{1} \subseteq \varphi\left(\left(U_{1}, U_{2}\right)\right) \subseteq U_{2}$ para todo $\left(U_{1}, U_{2}\right) \in \mathcal{P}$. 
Afirmamos que $\varphi[\mathcal{P}] \subseteq \mathcal{B}$ é uma base de abertos para $X$; isto concluirá a demonstração, uma vez que $|\varphi[\mathcal{P}]| \leq|\mathcal{P}| \leq|\mathcal{U} \times \mathcal{U}| \leq w(X)$ - esta última desigualdade decorre do fato de que, se $\mathcal{U}$ é finito, então $|\mathcal{U} \times \mathcal{U}|<\omega \leq w(X)$ e, se $\mathcal{U}$ é infinito, então $|\mathcal{U} \times \mathcal{U}|=|\mathcal{U}| \leq w(X)$.

De fato, tome $x \in X$ e $\Omega \in \tau$ quaisquer e suponha que $x \in \Omega$. Como $\mathcal{B}$ e $\mathcal{U}$ são bases para $X$, existem $U_{1}, U_{2} \in \mathcal{U}$ e $W \in \mathcal{B}$ tais que $x \in U_{1} \subseteq W \subseteq U_{2} \subseteq \Omega$. Assim, $\left(U_{1}, U_{2}\right) \in \mathcal{P}$, $\operatorname{logo}$ $\varphi\left(\left(U_{1}, U_{2}\right)\right) \in \varphi[\mathcal{P}]$ satisfaz $x \in \varphi\left(\left(U_{1}, U_{2}\right)\right) \subseteq \Omega$.

q.e.d.

A demonstração a seguir para o teorema 2.2.20 encontra-se em [5] $]^{14}$ e utiliza técnicas de submodelos elementares desenvolvidas na seção 1.1.3:

Demonstração do teorema 2.2.20. Como $w$ é monótona, basta provar que $w$ reflete todo cardinal sucessor, e o resultado seguirá da proposição 2.1.2(ii) e do lema 2.1.3.

Fixe então um cardinal sucessor $\kappa^{+}$arbitrário e seja $(X, \tau)$ um espaço topológico tal que $w(Y) \leq \kappa$ para todo $Y \in[X]^{\leq \kappa^{+}}$. Tome agora um submodelo elementar $\kappa$-covering $M \prec H_{\theta}$ satisfazendo $\{X, \tau, \kappa\} \subseteq M, \kappa \subseteq M$ e $|M|=\kappa^{+}$.

Afirmação: $\tau \cap M$ induz uma base para a topologia de subespaço em $X \cap M$.

Para demonstrar a afirmação, tome $x \in X \cap M$ e $U \in \tau$ arbitrários com $x \in U$. Por hipótese, $w((X \cap M) \backslash U) \leq \kappa$, logo existe $D \subseteq(X \cap M) \backslash U$ denso tal que $|D| \leq \kappa$. Pela propriedade $\kappa$-covering, existe $D^{\prime} \in M$ tal que $\left|D^{\prime}\right| \leq \kappa-$ logo, pelo lema 1.1.4, $D^{\prime} \subseteq M-$ e $D \subseteq D^{\prime}$; podemos supor que $D^{\prime} \subseteq X$, tomando, se necessário, $D^{\prime} \cap X \in M$ em seu lugar.

Por hipótese, temos que $w\left(D^{\prime} \cup\{x\}\right) \leq \kappa ;$ assim,

$$
H_{\theta} \models \exists \mathcal{B}\left(\mathcal{B} \text { é uma base para } D^{\prime} \cup\{x\} \text { e }|\mathcal{B}| \leq \kappa\right)
$$

\footnotetext{
${ }^{14} \mathrm{O}$ resultado demonstrado em [5] é, na verdade, a afirmação de que $w$ reflete $\omega_{1}$; no entanto, a prova aqui exibida é, mutatis mutandis, a mesma.
} 
e, portanto, pelo critério de Tarski, existe $\mathcal{B} \in M$ que é base para $D^{\prime} \cup\{x\}$ tal que $|\mathcal{B}| \leq \kappa-$ novamente pelo lema 1.1.4, $\mathcal{B} \subseteq M$.

Como $\mathcal{B}$ é base para $D^{\prime} \cup\{x\}$, existe $V \in \mathcal{B}$ satisfazendo $x \in V \subseteq U \cap\left(D^{\prime} \cup\{x\}\right)$; da definição de topologia de subespaço, segue então que existe $W \in \tau$ tal que $W \cap\left(D^{\prime} \cup\right.$ $\{x\})=V$ - pelo critério de Tarski, podemos supor que $W \in \tau \cap M$, pois $\left\{D^{\prime}, x\right\} \subseteq M$ e $V \in \mathcal{B} \subseteq M$.

Finalmente, $D^{\prime} \backslash V=D^{\prime} \backslash W$, pois $V=\left(W \cap D^{\prime}\right) \cup\{x\}$; assim, segue de $D \subseteq D^{\prime} \backslash$ $V=D^{\prime} \backslash W$ que $\bar{D} \subseteq \overline{D^{\prime} \backslash W}$. Agora, $(X \cap M) \backslash U \subseteq \bar{D}$ e, como $W$ é aberto em $X$, tem-se que $\overline{D^{\prime} \backslash W} \subseteq X \backslash W$. Portanto, $(X \cap M) \backslash U \subseteq X \backslash W$, de modo que $(X \cap M) \backslash U \subseteq(X \cap M) \backslash W$, o que implica que $x \in W \cap(X \cap M) \subseteq U \cap(X \cap M)$, como desejado - pois $W \in \tau \cap M$.

Assim, como $\tau \cap M$ induz uma base para a topologia de subespaço em $X \cap M$ e, por hipótese, $w(X \cap M) \leq \kappa$, segue do lema 2.2.21 que existe $\mathcal{U} \subseteq \tau \cap M$ com $|\mathcal{U}| \leq \kappa$ que induz uma base em $X \cap M$. Pela propriedade $\kappa$-covering, podemos supor que $\mathcal{U} \in M$ - pois, se $\mathcal{U}^{\prime} \in M$ é tal que $\mathcal{U} \subseteq \mathcal{U}^{\prime}$ e $\left|\mathcal{U}^{\prime}\right| \leq \kappa$, então $\mathcal{U}^{\prime} \cap \tau \in M$ contém $\mathcal{U}$ e também induz uma base em $X \cap M$; além disso, como $\left|\mathcal{U}^{\prime} \cap \tau\right| \leq \kappa$, segue do lema 1.1.4 que $\mathcal{U}^{\prime} \cap \tau \subseteq M$, logo $\mathcal{U}^{\prime} \cap \tau \subseteq \tau \cap M$, como requerido.

Temos então que

$\exists \mathcal{U} \in M(\mathcal{U} \subseteq \tau \cap M$ e $|\mathcal{U}| \leq \kappa$ e $\forall x \in X \cap M \forall \Omega \in \tau$

$$
\left.\left(x \in \Omega \Rightarrow \exists \Omega^{\prime} \in \mathcal{U}\left(x \in \Omega^{\prime} \text { e } \Omega^{\prime} \cap(X \cap M) \subseteq \Omega \cap(X \cap M)\right)\right)\right)
$$

e, como $\mathcal{U} \subseteq M$, isto implica que

$\exists \mathcal{U} \in M(\mathcal{U} \cap M \subseteq \tau \cap M$ e $|\mathcal{U}| \leq \kappa$ e $\forall x \in X \cap M \forall \Omega \in \tau \cap M$

$$
\left.\left(x \in \Omega \Rightarrow \exists \Omega^{\prime} \in \mathcal{U} \cap M\left(x \in \Omega^{\prime} \text { e } \Omega^{\prime} \cap M \subseteq \Omega \cap M\right)\right)\right),
$$

ou seja, $M \models w(X) \leq \kappa$. Como $M \prec H_{\theta}$, tem-se então que $H_{\theta} \models w(X) \leq \kappa$, como desejado.

q.e.d. 


\subsection{Funções cardinais locais}

Trataremos agora das funções cardinais $t, \chi$ e $\psi$. O primeiro fato a ser notado é que, para obter resultados positivos, é preciso restringir bastante a classe de espaços topológicos a considerar.

Proposição 2.3.1. Seja $\kappa$ um cardinal não-enumerável. Então as funções cardinais t, $\chi$ e $\psi$ não satisfazem $I U(\kappa)-e$, portanto, não refletem $\kappa$ fortemente - para a classe dos espaços de Hausdorff paracompactos e hereditariamente normais. ${ }^{15}$

Demonstração. Considere, em $X=\left[0, \kappa^{+}\right]$, a topologia em que todo $\alpha \in \kappa^{+}$é ponto isolado e \{]$\left.\left.\alpha, \kappa^{+}\right] \mid \alpha \in \kappa^{+}\right\}$é sistema fundamental de vizinhanças abertas para $\kappa^{+}$. Note que $X$ é um espaço de Hausdorff paracompacto e hereditariamente normal.

Defina, agora, $X_{\alpha}=[0, \alpha] \cup\left\{\kappa^{+}\right\}$para todo $\alpha \in \kappa^{+}$. Como todo $X_{\alpha}$ é discreto, tem-se que $t\left(X_{\alpha}\right)=\chi\left(X_{\alpha}\right)=\psi\left(X_{\alpha}\right)=\omega<\kappa$. Por outro lado, $t\left(\kappa^{+}, X\right)=\chi\left(\kappa^{+}, X\right)=\psi\left(\kappa^{+}, X\right)=\kappa^{+}>$ $\kappa$. Portanto, $I U(\kappa)$ não se verifica, uma vez que $\kappa^{+}>\kappa$ é regular e $X=\bigcup_{\alpha \in \kappa^{+}} X_{\alpha}$. q.e.d.

No entanto, resultados são obtidos ao considerarmos a classe dos espaços de Hausdorff compactos, como veremos no restante deste capítulo.

\subsubsection{Reflexão de $t$}

Iniciaremos esta seção introduzindo um conceito que será essencial para a mesma.

Definição 2.3.2. Sejam $(X, \tau)$ um espaço topológico e $\eta \neq \emptyset$ um ordinal. Uma seqüência $\left(x_{\alpha}\right)_{\alpha \in \eta}$ em $X$ é dita uma seqüência livre em $(X, \tau)$ se, e somente se, $\overline{\left\{x_{\alpha} \mid \alpha \in \beta\right\}} \cap$ $\overline{\left\{x_{\alpha} \mid \alpha \in[\beta, \eta[\}\right.}=\emptyset$ para todo $\beta \in \eta$.

\footnotetext{
${ }^{15}$ Note que, assim sendo, $t, \chi$ e $\psi$ não refletem $\kappa$ para tal classe, pois são funções cardinais monótonas vide 2.1.2(ii).
} 
Note que, em particular, a imagem de toda seqüência livre é um subespaço discreto do espaço original.

O resultado principal sobre as propriedades de reflexão da função cardinal $t$ seguirá como uma consequiência do seguinte teorema, devido a A. Arhangel'skiü:

Teorema 2.3.3. Sejam $\kappa$ um cardinal infinito e $X$ um espaço topológico $T_{3}$ e inicialmente- $\kappa$ compacto. Se $t(X) \geq \kappa^{+}$, então existe uma seqüência livre em $X$ de comprimento $\kappa^{+}$.

A demonstração deste teorema que apresentaremos foi retirada de [23] 3.12, e faz uso do seguinte lema:

Lema 2.3.4. Sejam $(X, \tau)$ um espaço topológico e $A, B \subseteq X$ tais que $A \cap \bar{B} \neq \emptyset$. Então existe $C \subseteq B$ satisfazendo $|C| \leq \chi(A, X)$ e $A \cap \bar{C} \neq \emptyset$.

Demonstração. Fixe $p \in A \cap \bar{B}$ arbitrário e seja $\mathcal{U} \subseteq \tau$ um sistema fundamental de vizinhanças para $A$ em $X$ tal que $|\mathcal{U}| \leq \chi(A, X)$. Para cada $U \in \mathcal{U}$, tem-se que $U \cap B \neq \emptyset$ - pois $p \in U$ é aderente a $B-$, de modo que se pode tomar $x_{U} \in U \cap B$. Assim, $C=\left\{x_{U} \mid U \in \mathcal{U}\right\} \subseteq B$ é tal que $|C| \leq|\mathcal{U}| \leq \chi(A, X)$ e $A \cap \bar{C} \neq \emptyset$ - pois, se ocorresse $A \subseteq X \backslash \bar{C} \in \tau$, deveria então existir $V \in \mathcal{U}$ tal que $V \subseteq X \backslash \bar{C}$, o que é um absurdo, dado que $x_{V} \in V$ e $x_{V} \in C \subseteq \bar{C}$. q.e.d.

Demonstração do teorema 2.3.3. Assuma que $t(X) \geq \kappa^{+} ; \operatorname{como} t(X)=\sup \{t(p, X) \mid p \in X\}$, temos que existe $p \in X$ tal que $t(p, X) \geq \kappa^{+}$. Fixe então $S \subseteq X$ tal que $p \in \bar{S}$ e $p \notin \bar{T}$ para todo $T \in[S]^{\leq \kappa}$, e defina $B=\bigcup\left\{\bar{T} \mid T \in[S]^{\leq \kappa}\right\}$.

Afirmação: Todo $E \in[B]^{\leq \kappa}$ satisfaz $\bar{E} \subseteq B$.

Para cada $x \in E$, tome $T_{x} \in[S]^{\leq \kappa}$ tal que $x \in \overline{T_{x}}$, e defina então $T=\bigcup\left\{T_{x} \mid x \in E\right\}$. Como $T \in[S]^{\leq \kappa}$, tem-se que $\bar{T} \subseteq B$. Agora, todo $x \in E$ verifica $x \in \overline{T_{x}} \subseteq \bar{T}$, $\operatorname{logo}$ $E \subseteq \bar{T}$ e, portanto, $\bar{E} \subseteq \bar{T} \subseteq B$. 
Construiremos seqüências $\left(x_{\alpha}\right)_{\alpha \in \kappa^{+}}$em $B$ e $\left(A_{\alpha}\right)_{\alpha \in \kappa^{+}}$em $\wp(X)$ tais que, para todo $\alpha \in \kappa^{+}$, são satisfeitas as sete condições seguintes:

(i) $A_{\alpha}$ é fechado em $X$;

(ii) $p \in A_{\alpha}$;

(iii) $A_{\alpha} \subseteq A_{\beta}$ para todo $\beta \in \alpha$;

(iv) $A_{\alpha} \subseteq X \backslash \overline{\left\{x_{\beta} \mid \beta \in \alpha\right\}}$

$(v)$ existe uma família de abertos $\left\{U_{\xi}^{\alpha} \mid \xi \in \kappa\right\}$ tal que

$$
A_{\alpha}=\bigcap_{\xi \in \kappa} U_{\xi}^{\alpha}=\bigcap_{\xi \in \kappa} \overline{U_{\xi}^{\alpha}}
$$

(vi) $\chi\left(A_{\alpha}, X\right) \leq \kappa ;$

(vii) $x_{\alpha} \in B \cap A_{\alpha}$;

Procederemos por indução transfinita. Suponha que, para um certo $\gamma \in \kappa^{+}$, tenhamos construído $\left(x_{\alpha}\right)_{\alpha \in \gamma}$ e $\left(A_{\alpha}\right)_{\alpha \in \gamma}$ de modo a satisfazer as condições de $(i)$ a (vii) para todo $\alpha \in \gamma$.

Considere $S_{\gamma}=\left\{x_{\alpha} \mid \alpha \in \gamma\right\}$. Como $S_{\gamma} \in[B] \leq \kappa$, então $p \notin \overline{S_{\gamma}}$ - pois $p \notin B$ e, pela afirmação provada no início da demonstração, $B \supseteq \overline{S_{\gamma}}$ - $\operatorname{logo}$, por $X$ ser $T_{3}$, existe $H_{\gamma} \subseteq X \backslash \overline{S_{\gamma}}$ que é $G_{\delta}$ e fechado em $X$ e que contém $p$ - para tanto, fixe um sistema fundamental de vizinhanças abertas $\mathcal{V}$ para $p$ em $X$, tome $V_{0}^{\gamma} \in \mathcal{V}$ tal que $\overline{V_{0}^{\gamma}} \subseteq X \backslash \overline{S_{\gamma}}$ e, para todo $n \in \omega$, tome $V_{n+1}^{\gamma} \in \mathcal{V}$ tal que $\overline{V_{n+1}^{\gamma}} \subseteq V_{0}^{\gamma}$; finalmente, seja $H_{\gamma}=\bigcap_{n \in \omega} V_{n}^{\gamma}=\bigcap_{n \in \omega} \overline{V_{n}^{\gamma}}$.

Defina então

$$
A_{\gamma}=H_{\gamma} \cap \bigcap_{\alpha \in \gamma} A_{\alpha}
$$

- no caso $\gamma=0$, defina apenas $A_{\gamma}=H_{\gamma}$. 
É imediato que $A_{\gamma}$ satisfaz as condições $(i),(i i),($ iii $)$ e (iv). Para a condição $(v)$, segue da hipótese de indução e do processo de obtenção de $H_{\gamma}$ que

$$
A_{\gamma}=H_{\gamma} \cap \bigcap_{\alpha \in \gamma} A_{\alpha}=\left(\bigcap_{n \in \omega} V_{n}^{\gamma}\right) \cap \bigcap_{\alpha \in \gamma}\left(\bigcap_{\xi \in \kappa} U_{\xi}^{\alpha}\right)=\left(\bigcap_{n \in \omega} \overline{V_{n}^{\gamma}}\right) \cap \bigcap_{\alpha \in \gamma}\left(\bigcap_{\xi \in \kappa} \overline{U_{\xi}^{\alpha}}\right) ;
$$

assim, como $\omega+|\gamma| \cdot \kappa \leq \omega+\kappa \cdot \kappa=\kappa$, basta tomar uma ordenação - com eventuais repetições, caso seja necessário -

$$
\left\{U_{\xi}^{\gamma} \mid \xi \in \kappa\right\}=\left\{V_{n}^{\gamma} \mid n \in \omega\right\} \cup \bigcup_{\alpha \in \gamma}\left\{U_{\xi}^{\alpha} \mid \xi \in \kappa\right\}
$$

Afirmamos agora que o conjunto

$$
\mathcal{U}=\left\{\bigcap_{\xi \in F} U_{\xi}^{\gamma} \mid \mathcal{F} \in[\kappa]^{<\omega}\right\}
$$

é um sistema fundamental de vizinhanças abertas para $A_{\gamma}$ em $X$ - o que, por sua vez, implica que $A_{\gamma}$ também satisfaz a condição $(v i)$, uma vez que $|\mathcal{U}|=\kappa$. De fato, tome $\Omega$ aberto em $X$ tal que $A_{\gamma} \subseteq \Omega$. Por $(v)$, temos que

$$
\bigcap_{\xi \in \kappa} \overline{U_{\xi}^{\gamma}}=A_{\gamma} \subseteq \Omega
$$

de modo que $\left\{X \backslash \overline{U_{\xi}^{\gamma}} \mid \xi \in \kappa\right\} \cup\{\Omega\}$ é um recobrimento aberto de $X$. Como $X$ é inicialmente$\kappa$-compacto, existe $F \in[\kappa]^{<\omega}$ tal que

$$
X=\Omega \cup \bigcup_{\xi \in F} X \backslash \overline{U_{\xi}^{\gamma}}
$$

i.e.,

$$
\bigcap_{\xi \in F} \overline{U_{\xi}^{\gamma}} \subseteq \Omega
$$

assim,

$$
A_{\gamma}=\bigcap_{\xi \in \kappa} U_{\xi}^{\gamma} \subseteq \bigcap_{\xi \in F} U_{\xi}^{\gamma} \subseteq \bigcap_{\xi \in F} \overline{U_{\xi}^{\gamma}} \subseteq \Omega
$$


como desejado.

Finalmente, note que $p \in A_{\gamma} \cap \bar{S} \subseteq A_{\gamma} \cap \bar{B}$ - pois $S \subseteq B$-; assim, pelo lema 2.3.4, existe $C \subseteq B$ tal que $|C| \leq \chi\left(A_{\gamma}, X\right) \leq \kappa$ e $A_{\gamma} \cap \bar{C} \neq \emptyset$. Tome então $x_{\gamma} \in A_{\gamma} \cap \bar{C}$ arbitrário. Pela afirmação 1 , temos que $\bar{C} \subseteq B$, o que implica que a condição (vii) também é satisfeita.

Tendo assim concluído a construção, temos que $\left(x_{\alpha}\right)_{\alpha \in \kappa^{+}}$é uma seqüência livre em $X$, pois, para todo $\alpha \in \kappa^{+}$,

$$
\overline{\left\{x_{\beta} \mid \beta \in\left[\alpha, \kappa^{+}[\}\right.\right.} \subseteq \overline{A_{\alpha}}=A_{\alpha} \subseteq X \backslash \overline{\left\{x_{\beta} \mid \beta \in \alpha\right\}} .
$$

q.e.d.

Podemos agora enunciar a principal propriedade de reflexão de $t$ - a demonstração que apresentamos foi extraída de $[18]^{16}$ —:

Teorema 2.3.5. Seja $\kappa$ um cardinal infinito. Então $t$ reflete $\kappa^{+}$fortemente para a classe dos espaços topológicos $T_{3}$ e inicialmente- $\kappa^{+}$-compactos.

Demonstração. Pela proposição 2.1.2(ii), basta provar que $t$ reflete $\kappa^{+}$para a classe dos espaços topológicos $T_{3}$ e inicialmente- $\kappa^{+}$-compactos - uma vez que $t$ é uma função cardinal monótona.

Seja $X$ um espaço topológico $T_{3}$ e inicialmente- $\kappa^{+}$-compacto e suponha que $t(X) \geq \kappa^{+}$. Pelo teorema 2.3.3, existe uma seqüência livre $\left(x_{\alpha}\right)_{\alpha \in \kappa^{+}}$em $X$. Como $X$ é inicialmente- $\kappa^{+}-$ compacto, então

$$
\bigcap_{\alpha \in \kappa^{+}} \overline{\left\{x_{\beta} \mid \beta \in\left[\alpha, \kappa^{+}[\}\right.\right.} \neq \emptyset ;
$$

assim sendo, tome $z \in \bigcap_{\alpha \in \kappa^{+}} \overline{\left\{x_{\beta} \mid \beta \in\left[\alpha, \kappa^{+}[\}\right.\right.}$e seja $Y=\{z\} \cup\left\{x_{\alpha} \mid \alpha \in \kappa^{+}\right\}$. Então $Y \in[X]^{\leq \kappa^{+}}$e $t(Y) \geq \kappa^{+}$, pois $t(z, Y) \geq \kappa^{+}$- de fato, $z \in \overline{\left\{x_{\alpha} \mid \alpha \in \kappa^{+}\right\}}$e, como a seqüência é livre, para todo $\alpha \in \kappa^{+}$tem-se que $z \notin \overline{\left\{x_{\beta} \mid \beta \in \alpha\right\}}$; assim, a regularidade de $\kappa^{+}$implica que, se $A \subseteq\left\{x_{\alpha} \mid \alpha \in \kappa^{+}\right\}$é tal que $|A| \leq \kappa$, então $z \notin \bar{A}$. q.e.d.

\footnotetext{
${ }^{16}$ Os enunciados originais dos teoremas 2.3.3 e 2.3.5 se referem a espaços de Hausdorff compactos; a hipótese original foi enfraquecida, uma vez que é suficiente assumir que os espaços topológicos considerados são $T_{3}$ e inicialmente- $\kappa$-compactos.
} 
Corolário 2.3.6. A função cardinal t reflete fortemente todos os cardinais infinitos para $\mathcal{C}_{2}$.

Demonstração. Segue do corolário anterior que, dado um cardinal infinito $\kappa$ arbitrário, $t$ reflete $\kappa^{+}$para $\mathcal{C}_{2}$. Assim, o resultado segue da proposição 2.1.2(ii) e do lema 2.1.3, pois $t$ é monótona.

q.e.d.

\subsubsection{Reflexão de $\chi$}

Já tendo o resultado 2.3.5, podemos agora demonstrar o principal teorema de reflexão da função cardinal $\chi$ :

Teorema 2.3.7. Seja $\kappa$ um cardinal infinito. Então $\chi$ reflete $\kappa^{+}$fortemente para a classe dos espaços topológicos $T_{3}$ e inicialmente- $\kappa^{+}$-compactos.

Para tanto, será necessário o seguinte lema:

Lema 2.3.8. Sejam $(X, \tau)$ um espaço topológico, $x \in X$ e $\mathcal{V}$ um sistema fundamental de vizinhanças abertas para $x$. Então existe $\mathcal{V}^{\prime} \subseteq \mathcal{V}$ tal que $\mathcal{V}^{\prime}$ é sistema fundamental de vizinhanças para $x$ e $\left|\mathcal{V}^{\prime}\right| \leq \chi(x, X)$.

Demonstração. Análoga à demonstração do lema 2.2.21.

q.e.d.

A demonstração que apresentaremos agora foi retirada de $[5]^{17}$, e mais uma vez é baseada em técnicas de submodelos elementares.

Demonstração do teorema 2.3.7. Como $\chi$ é uma função cardinal monótona, é suficiente provar que $\chi$ reflete $\kappa^{+}$para a classe dos espaços topológicos $T_{3}$ e inicialmente- $\kappa^{+}$-compactos.

Seja $(X, \tau)$ um espaço $T_{3}$ e inicialmente- $\kappa^{+}$-compacto tal que $\chi(Y) \leq \kappa$ para todo $Y \in$ $[X]^{\leq \kappa^{+}}$. Então $t(Y) \leq \kappa$ para todo $Y \in[X]^{\leq \kappa^{+}}$; logo, pelo teorema 2.3.5, tem-se que $t(X) \leq \kappa$.

\footnotetext{
${ }^{17}$ Assim como no teorema 2.2.20, o resultado demonstrado em [5] refere-se a $\omega_{1}$; no entanto, uma demonstração análoga se aplica ao caso geral.
} 
Seja $M$ um submodelo elementar $\kappa$-covering de $H_{\theta}$ tal que $\{X, \tau, \kappa\} \cup \kappa \subseteq M$ e $|M| \leq \kappa^{+}$. Afirmamos que $\tau \cap M$ induz uma base para a topologia de subespaço em $X \cap M$.

Suponha, por absurdo, que existam $x \in X \cap M$ e $U \in \tau_{x}$ tais que $(T \cap M) \backslash U \neq \emptyset$ para todo $T \in \tau_{x} \cap M$. Isto implica que existe $z \in \bigcap\left\{\overline{(T \cap M) \backslash U} \mid T \in \tau_{x} \cap M\right\}$, pois $(X, \tau)$ é inicialmente- $\kappa^{+}$-compacto. Assim, em particular, $z \in \overline{(X \cap M) \backslash U}$; como $t(X) \leq \kappa$, existe então $D \subseteq(X \cap M) \backslash U$ tal que $|D| \leq \kappa$ e $z \in \bar{D}$. Pela propriedade $\kappa$-covering de $M$, existe $D^{\prime} \in M$ tal que $\left|D^{\prime}\right| \leq \kappa$ e $D \subseteq D^{\prime} \subseteq X$ - caso $D^{\prime} \nsubseteq X$, tome $D^{\prime} \cap X \in M$ em seu lugar.

Por hipótese, $\chi\left(D^{\prime} \cup\{x\}\right) \leq \kappa$; logo, pelo critério de Tarski, existe $\mathcal{B}_{x} \in M$ tal que $\mathcal{B}_{x} \subseteq \tau_{x}$, $\left|\mathcal{B}_{x}\right| \leq \kappa$ - portanto, pelo lema $1.1 .4, \mathcal{B}_{x} \subseteq M-$ e $\mathcal{B}_{x}$ induz um sistema fundamental de vizinhanças para $x$ em $D^{\prime} \cup\{x\}$. Assim, existe $V \in \mathcal{B}_{x} \subseteq M$ tal que $x \in V$ e $V \cap D^{\prime} \subseteq U \cap D^{\prime}$, $\operatorname{logo} D \subseteq D^{\prime} \backslash U \subseteq D^{\prime} \backslash V \subseteq X \backslash V$, o que implica que $V \cap D=\emptyset$. Como $(X, \tau) \in \mathcal{T}_{3}$ e $x \in V \in \tau \cap M$, segue novamente do critério de Tarski ${ }^{18}$ que existe $W \in \tau_{x} \cap M$ tal que $\bar{W} \subseteq V \subseteq X \backslash D$; por $V$ ser aberto, tem-se que $V \subseteq \operatorname{int}(X \backslash D)=X \backslash \bar{D}, \operatorname{logo} \bar{W} \cap \bar{D}=\emptyset$. Mas isto é uma contradição, pois $z \in \bar{D}$ e $z \in \overline{(W \cap M) \backslash U} \subseteq \bar{W}$.

Assim, $\{U \cap M \mid U \in \tau \cap M\}$ é uma base para a topologia de subespaço em $X \cap M$. Como, por hipótese, $\chi(X \cap M) \leq \kappa$, segue do lema 2.3.8 que

$$
\forall x \in X \cap M \exists \mathcal{V}_{x}\left(\mathcal{V}_{x} \subseteq \tau \cap M,\left|\mathcal{V}_{x}\right| \leq \kappa \text { e } \forall T \in \tau\left(x \in T \Rightarrow \exists A \in \mathcal{V}_{x}(A \cap M \subseteq T \cap M)\right)\right)
$$

Para $x \in X \cap M$ arbitrário, temos então pela propriedade $\kappa$-covering - uma vez que $\mathcal{V}_{x} \subseteq M$ e $\left|\mathcal{V}_{x}\right| \leq \kappa-$ que existe $\mathcal{V}_{x}^{\prime} \in M$ tal que $\mathcal{V}_{x} \subseteq \mathcal{V}_{x}^{\prime}$ e $\left|\mathcal{V}_{x}^{\prime}\right| \leq \kappa, \operatorname{logo} \mathcal{V}_{x}^{\prime} \cap \tau \in M$ também induz um sistema fundamental de vizinhanças para $x \in X \cap M$ em $X \cap M$. Como $\left|\mathcal{V}_{x}^{\prime} \cap \tau\right| \leq \kappa$,

${ }^{18}$ Note que a função

$$
\begin{aligned}
F: \wp(X) & \rightarrow \wp(X) \\
A & \mapsto \bar{A}
\end{aligned}
$$

é um elemento de $M$, por uma aplicação do critério de Tarski a

$$
\exists F \in{ }^{\wp(X)} \wp(X) \forall A \in \wp(X) \forall y \in X\left(y \in F(A) \Leftrightarrow \forall U \in \tau_{y}(U \cap A \neq \emptyset)\right)
$$

assim, nesta passagem, pode-se proceder de modo semelhante ao feito na demonstração do lema 1.1.14(b) — vide nota de rodapé na mesma. 
segue do lema 1.1.4 que $\mathcal{V}_{x}^{\prime} \cap \tau \subseteq \tau \cap M$, de modo que podemos tomar $\mathcal{V}_{x}^{\prime} \cap \tau \in M$ no lugar de $\mathcal{V}_{x}$ - ou, simplesmente, podemos supor que $\mathcal{V}_{x} \in M$ na fórmula anterior.

Segue então que

$\forall x \in X \cap M \exists \mathcal{V}_{x} \in M\left(\mathcal{V}_{x} \cap M \subseteq \tau \cap M,\left|\mathcal{V}_{x}\right| \leq \kappa\right.$ e $\forall T \in \tau \cap M$

$$
\left.\left(x \in T \Rightarrow \exists A \in \mathcal{V}_{x} \cap M(A \cap M \subseteq T \cap M)\right)\right),
$$

i.e., $M \models \chi(X) \leq \kappa$. Portanto, por elementaridade, $H_{\theta} \models \chi(X) \leq \kappa$.

q.e.d.

Corolário 2.3.9. A função cardinal $\chi$ reflete fortemente todos os cardinais infinitos para $\mathcal{C}_{2}$.

Demonstração. Análoga à demonstração de 2.3.6 a partir de 2.3.5.

q.e.d.

Façamos agora uma observação — presente em [5] — sobre a necessidade da hipótese de $(X, \tau)$ ser um espaço $T_{3}$ no teorema 2.3.7. Temos que é consistente que o resultado seja verdadeiro se trocarmos a hipótese " $(X, \tau) \in \mathcal{T}_{3}$ " por " $(X, \tau) \in \mathcal{T}_{2}$ e $t(X) \leq \kappa$ ", fato que decorre da seguinte afirmação:

Proposição 2.3.10. Sejam $\kappa$ um cardinal infinito e $(X, \tau)$ um espaço de Hausdorff inicialmente- $2^{\kappa}$-compacto tal que $t(X) \leq \kappa$. Então $(X, \tau)$ é compacto.

Demonstração. Suponha, por absurdo, que $(X, \tau)$ não seja compacto. Existe então uma família $\mathscr{F}$ de fechados em $(X, \tau)$ com a propriedade da intersecção finita tal que $\bigcap \mathscr{F}=\emptyset$; assim, pelo lema de Kuratowski-Zorn, existe um filtro maximal de fechados em $(X, \tau)$ - digamos, $\mathscr{G}$ - com $\mathscr{F} \subseteq \mathscr{G}$. Fazendo uso do lema 1.1.10, tome então $M \prec H_{\theta} \kappa$-fechado tal que $\{X, \tau, \mathscr{G}\} \subseteq M$ e $|M| \leq 2^{\kappa}$.

Afirmamos que a família $\{\overline{F \cap M} \mid F \in \mathscr{G} \cap M\}$ tem a propriedade da intersecção finita. De fato, se houvesse $\mathscr{G}^{\prime} \subseteq \mathscr{G} \cap M$ finito e não-vazio tal que $\bigcap\left\{\overline{F \cap M} \mid F \in \mathscr{G}^{\prime}\right\}=\emptyset$, teríamos que $\emptyset=\bigcap\left\{F \cap M \mid F \in \mathscr{G}^{\prime}\right\}=\left(\bigcap_{\mathscr{G}^{\prime}}\right) \cap M$, de modo que $\forall x \in M\left(x \notin \bigcap^{\mathscr{G}^{\prime}}\right)$, i.e., $M \models \bigcap \mathscr{G}^{\prime}=\emptyset$ - note que, como $\mathscr{G}^{\prime} \subseteq M$ é finito, então $\mathscr{G}^{\prime} \in M$-; isto é um absurdo, pois $M \prec H_{\theta}$ e $\mathscr{G} \in M$ é um filtro. 
Considere $F_{M}=\bigcap\{\overline{F \cap M} \mid F \in \mathscr{G} \cap M\} ;$ como $|\mathscr{G} \cap M| \leq 2^{\kappa}$ e $(X, \tau)$ é inicialmente- $2^{\kappa}$ compacto, tem-se que $F_{M} \neq \emptyset$. Fixe então $z \in F_{M}$ arbitrário.

Provaremos agora que $\{\overline{F \cap M} \mid F \in \mathscr{G} \cap M\} \subseteq \mathscr{G}$. Para tanto, tome $F \in \mathscr{G} \cap M$ arbitrário; como $t(X) \leq \kappa$, existe $D_{F} \subseteq F \cap M$ tal que $z \in \overline{D_{F}}$ e $\left|D_{F}\right| \leq \kappa-\operatorname{logo} D_{F} \in M$, pois $[M]^{\leq \kappa} \subseteq M$. Agora, para cada $G \in \mathscr{G} \cap M$, tem-se que $z \in \overline{D_{F}} \cap \overline{G \cap M} \subseteq \overline{D_{F}} \cap \bar{G}=$ $\overline{D_{F}} \cap G$, logo $H_{\theta} \models \exists y \in \overline{D_{F}} \cap G$ e, do critério de Tarski, segue que existe $y \in M$ tal que $y \in \overline{D_{F}} \cap G$. Assim, $M \models \forall G \in \mathscr{G}\left(\overline{D_{F}} \cap G \neq \emptyset\right)$, e tem-se então por elementaridade que $H_{\theta} \models \forall G \in \mathscr{G}\left(\overline{D_{F}} \cap G \neq \emptyset\right)$. Isto implica que $\overline{D_{F}} \in \mathscr{G}$ - pois $\mathscr{G}$ é maximal - e, portanto, $\overline{F \cap M} \in \mathscr{G}$.

Podemos, com isso, concluir que $F_{M} \in \mathscr{G}$. De fato, suponha, por absurdo, que $F_{M} \notin \mathscr{G}$; como $\mathscr{G}$ é maximal, deve então existir $H \in \mathscr{G}$ tal que $F_{M} \cap H=\emptyset$. Mas então $\emptyset=F_{M} \cap H=$ $(\bigcap\{\overline{F \cap M} \mid F \in \mathscr{G} \cap M\}) \cap H=\bigcap\{\overline{F \cap M} \cap H \mid F \in \mathscr{G} \cap M\}$, o que é um absurdo, pois $(X, \tau)$ é inicialmente-2 ${ }^{\kappa}$-compacto, $\{\overline{F \cap M} \cap H \mid F \in \mathscr{G} \cap M\}$ é uma família de fechados com a propriedade da interseç̧ão finita - uma vez que $\{\overline{F \cap M} \mid F \in \mathscr{G} \cap M\} \subseteq \mathscr{G}$ e $H \in \mathscr{G}$ - e $|\mathscr{G} \cap M| \leq 2^{\kappa}$.

Finalmente, como $\bigcap \mathscr{G}=\emptyset$, tem-se que $F_{M} \neq\{z\}$ - do contrário, como $F_{M} \in \mathscr{G}$, ocorreria $\mathscr{G}=\{F \subseteq X \mid F$ é fechado em $(X, \tau)$ e $z \in F\}$, implicando que $\bigcap \mathscr{G}=\{z\}$, uma contradição. Tome então $z^{\prime} \in F_{M} \backslash\{z\}$ e $U, U^{\prime} \in \tau$ tais que $z \in U, z^{\prime} \in U^{\prime}$ e $U \cap U^{\prime}=\emptyset$. Dado $F \in \mathscr{G} \cap M$ arbitrário, tem-se que $z \in \overline{(F \cap M) \cap U}$, logo - como $t(X) \leq \kappa-$ existe $D \subseteq F \cap M \cap U$ tal que $|D| \leq \kappa$ e $z \in \bar{D}$; da mesma forma, existe $D^{\prime} \subseteq F \cap M \cap U^{\prime}$ com $\left|D^{\prime}\right| \leq \kappa$ e $z^{\prime} \in \overline{D^{\prime}}-$ note que $D, D^{\prime} \in M$, pois $M$ é $\kappa$-fechado. Agora, pelo mesmo argumento utilizado no quarto parágrafo desta demonstração para provar que $\overline{D_{F}} \in \mathscr{G}$, podemos concluir que $\bar{D}, \overline{D^{\prime}} \in \mathscr{G}$. Assim sendo, $\overline{D^{\prime}} \in \mathscr{G} \cap M, \log z \in \overline{\overline{D^{\prime}} \cap M} \subseteq \overline{\overline{D^{\prime}}}=\overline{D^{\prime}}$; isto é um absurdo, pois $z \in U, U \in \tau$ e $U \cap D^{\prime}=\emptyset$.

q.e.d.

De fato, assumindo-se a hipótese de que $2^{\kappa}=\kappa^{+}$, obtemos:

Corolário 2.3.11. Seja $\kappa$ um cardinal infinito. Suponha que $2^{\kappa}=\kappa^{+}$. Então $\chi$ reflete $\kappa^{+}$fortemente para a classe dos espaços de Hausdorff inicialmente- $\kappa^{+}$-compactos tais que 
$t(X) \leq \kappa$.

Demonstração. Seja $(X, \tau) \in \mathcal{T}_{2}$ inicialmente- $\kappa^{+}$-compacto tal que $t(X) \leq \kappa$. Pela proposição 2.3.10, tem-se que $(X, \tau) \in \mathcal{C}_{2}, \operatorname{logo}(X, \tau)$ é regular. A afirmação decorre então do teorema 2.3.7.

q.e.d.

\subsubsection{Reflexão de $\psi$}

Passemos agora à função cardinal $\psi$. Antes de mais nada, notemos o seguinte fato:

Proposição 2.3.12. Sejam $\kappa$ um cardinal infinito $e(X, \tau)$ um espaço topológico $T_{3}$ e inicial-

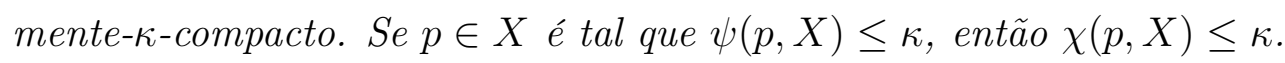

Demonstração. Suponha que $p \in X$ verifica $\psi(p, X) \leq \kappa$ e tome $\mathscr{C} \subseteq \tau$ tal que $|\mathscr{C}| \leq \kappa$ e $\bigcap \mathscr{C}=\{p\}$. Como $(X, \tau)$ verifica $T_{3}$, então para cada $U \in \mathscr{C}$ existe $V_{U} \in \tau$ satisfazendo $p \in V_{U} \subseteq \overline{V_{U}} \subseteq U$.

Afirmamos que $\mathcal{V}=\left\{\bigcap_{U \in \mathscr{C}^{\prime}} V_{U} \mid \mathscr{C}^{\prime} \in[\mathscr{C}]^{<\omega} \backslash\{\emptyset\}\right\}$ é um sistema fundamental de vizinhanças para $p$ em $(X, \tau)$ - o que nos permite então concluir o desejado, uma vez que $\left|[\mathscr{C}]^{<\omega} \backslash\{\emptyset\}\right|=|\mathscr{C}| \leq \kappa$.

De fato, tome $\Omega \in \tau_{p}$ arbitrário. Se $\Omega=X$, é imediato que todo $V \in \mathcal{V}$ satisfaz $p \in V \subseteq \Omega$; suponha então $\Omega \neq X$. Temos que $\bigcap_{U \in \mathscr{C}} \overline{V_{U}} \subseteq \bigcap \mathscr{C}=\{p\}$, de modo que $\Omega \cup \bigcup_{U \in \mathscr{C}}\left(X \backslash \overline{V_{U}}\right)=$ $X$; logo, por ser $(X, \tau)$ inicialmente- $\kappa$-compacto, existe $\mathscr{C}^{\prime} \subseteq \mathscr{C}$ finito tal que $\Omega \cup \bigcup_{U \in \mathscr{C}^{\prime}}(X \backslash$ $\left.\overline{V_{U}}\right)=X$ - note que $\mathscr{C}^{\prime} \neq \emptyset$, pois $\Omega \neq X$. Assim, $p \in \bigcap_{U \in \mathscr{C}^{\prime}} V_{U} \subseteq \bigcap_{U \in \mathscr{C}^{\prime}} \overline{V_{U}} \subseteq \Omega$ e, como $\bigcap_{U \in \mathscr{C}}, V_{U} \in \mathcal{V}$, temos o afirmado.

q.e.d.

Resultados afirmativos em ZFC sobre propriedades de reflexão de $\psi$ são desconhecidos até o momento. Exibiremos aqui um resultado de consistência, baseado no seguinte teorema enunciado em $[24]^{19}$; esta demonstração do mesmo deve-se a O. T. Alas -:

\footnotetext{
${ }^{19}$ No caso $\kappa=\omega$ e com a hipótese mais forte $(X, \tau) \in \mathcal{C}_{2}$.
} 
Teorema 2.3.13. Sejam $\kappa$ um cardinal infinito $e(X, \tau)$ um espaço topológico $T_{3}$ e inicialmente- $\kappa^{+}$-compacto. Suponha que $\psi(Y) \leq \kappa$ para todo $Y \in[X]^{\leq 2^{\kappa}}$. Então $\psi(X) \leq \kappa$.

Demonstração. Tome $Y \in[X]^{\leq 2^{\kappa}}$ arbitrário. Construiremos, usando indução transfinita, uma seqüência crescente $\left(Y_{\alpha}\right)_{\alpha \in \kappa^{+}}$em $[X]^{\leq 2^{\kappa}}$ da seguinte maneira:

$\cdot Y_{0}=Y$.

- Em posse de $Y_{\alpha}$ para $\alpha \in \kappa^{+}$dado, fixe, para cada $A \in\left[Y_{\alpha}\right]^{\leq \kappa}$, um elemento $y_{A} \in X$ que é ponto de acumulação completo de $A$ em $(X, \tau)$ — pela proposição 1.2.5, um tal $y_{A}$ existe, pois, por hipótese, $(X, \tau)$ é inicialmente- $\kappa$-compacto. Defina então $Y_{\alpha+1}=$ $Y_{\alpha} \cup\left\{y_{A} \mid A \in\left[Y_{\alpha}\right]^{\leq \kappa}\right\}$ - note que, como $\left|Y_{\alpha}\right| \leq 2^{\kappa}$, então $\left|\left[Y_{\alpha}\right]^{\leq \kappa}\right| \leq 2^{\kappa}$, de modo que $\left|Y_{\alpha+1}\right| \leq 2^{\kappa}$.

- Suponha que, para um certo $\alpha \in \kappa^{+}$limite, já tenhamos construído $\left(Y_{\beta}\right)_{\beta \in \alpha}$. Nesse caso, defina $Y_{\alpha}=\bigcup_{\beta \in \alpha} Y_{\beta}$ - que é um elemento de $[X]^{\leq 2^{\kappa}}$, uma vez que $|\alpha| \leq \kappa$.

Considere agora $Z=\bigcup_{\alpha \in \kappa^{+}} Y_{\alpha}$. Tome $A \in[Z]^{\leq \kappa}$ arbitrário. Como $\kappa^{+}$é regular, existe $\alpha \in \kappa^{+}$tal que $A \subseteq Y_{\alpha}$; logo, por construção, existe $y_{A} \in Y_{\alpha+1} \subseteq Z$ que é ponto de acumulação completo de $A$ em $(X, \tau)$ — e, portanto, em $Z$. Assim, como $A$ é arbitrário, tem-se — pela proposição 1.2.5 - que $Z$ é inicialmente- $\kappa$-compacto. Além disso, $|Z| \leq 2^{\kappa} \cdot \kappa^{+} \leq 2^{\kappa} \cdot 2^{\kappa}=2^{\kappa}$, logo - por hipótese - $\psi(Z) \leq \kappa$.

Sendo $Z$ um espaço inicialmente- $\kappa$-compacto e $T_{3}$ tal que $\psi(Z) \leq \kappa$, tem-se pela proposição 2.3.12 que $\chi(Z) \leq \kappa$, o que implica que $\chi(Y) \leq \kappa$, uma vez que a função cardinal $\chi$ é monótona e $Y \subseteq Z$. Segue então que $\chi(Y) \leq \kappa$ para todo $Y \in[X]^{\leq 2^{\kappa}}$; assim, pelo teorema 2.3.7, tem-se que $\chi(X) \leq \kappa, \log$ $\psi(X) \leq \chi(X) \leq \kappa$.

q.e.d.

Corolário 2.3.14. Seja $\kappa$ um cardinal infinito e suponha que $2^{\kappa}=\kappa^{+}$. Então $\psi$ reflete $\kappa^{+}$ fortemente para a classe dos espaços topológicos $T_{3}$ e inicialmente- $\kappa^{+}$-compactos.

Demonstração. Decorre imediatamente do teorema anterior e da proposição 2.1.2(ii). q.e.d. 
Corolário 2.3.15. Assuma GCH. Então a função cardinal $\psi$ reflete fortemente todos os cardinais infinitos para $\mathcal{C}_{2}$.

Demonstração. Também análoga à demonstração de 2.3.6 a partir de 2.3.5.

q.e.d.

\subsection{Problemas em aberto}

Concluiremos este capítulo com alguns comentários sobre problemas que permanecem em aberto sobre os tópicos abordados no mesmo.

A primeira pergunta diz respeito à densidade; mais precisamente, à necessidade de se assumir GCH no corolário 2.2.8, levando-se em conta os teoremas 2.2.4 e 2.2.10.

Pergunta 1. Seja $\lambda$ um cardinal tal que $\lambda \neq c f(\lambda)>\omega$. É consistente que $d$ não reflita $\lambda$ $\operatorname{para} \mathcal{T}_{2}$ ?

No que diz respeito a $L$, pode-se também questionar a necessidade de hipóteses de teoria dos conjuntos nos resultados obtidos — neste caso, o corolário 2.2.18, tendo-se em conta o teorema 2.2.13 e o corolário 2.2.17.

Pergunta 2. Seja $\kappa>\omega$ um cardinal limite. É consistente que L não reflita $\kappa$ para $\mathcal{T}_{2}$ ?

Pergunta 3. Seja $\lambda$ um cardinal singular. É consistente que $L$ não reflita $\lambda$ para $\mathcal{T}_{2}$ ?

Passando agora às funções cardinais locais, podemos começar com uma pergunta que já foi sugerida no texto:

Pergunta 4. Pode-se enfraquecer a hipótese de $X$ ser um espaço $T_{3}$ no teorema 2.3.7?

E, uma vez que não há resultados sobre propriedades de reflexão de $\psi$ que não sejam de consistência, é natural também questionar a necessidade de se assumir GCH no corolário 2.3.15; como tal hipótese foi considerada em virtude da hipótese $2^{\kappa}=\kappa^{+}$no corolário 2.3.14, a pergunta pode ser formulada da seguinte maneira: 
Pergunta 5. Seja $\kappa$ um cardinal infinito. É consistente que $\psi$ não reflita $\kappa^{+}$para a classe dos espaços topológicos $T_{3}$ e inicialmente- $\kappa$-compactos?

Na verdade, como é salientado em [24], tal pergunta encontra-se em aberto mesmo sob hipóteses mais fortes - e ainda que se restrinja ao caso particular $\kappa=\omega-$ :

Pergunta 6. É consistente que $\psi$ não reflita $\omega_{1}$ para $\mathcal{C}_{2}$ ? 


\section{Capítulo 3}

\section{Reflexão da metrizabilidade}

\subsection{O problema de Hamburger}

Os tópicos abordados neste capítulo estão relacionados ao problema de Hamburger, nome pelo qual ficou conhecida a seguinte pergunta ${ }^{1}$ atribuída a P. Hamburger — vide, e.g., [15] —:

Existe algum espaço de Hausdorff $X$ não-metrizável e de caráter enumerável tal que todo $Y \in[X]^{\leq \omega_{1}}$ é metrizável?

Note que, se a hipótese $\chi(X)=\omega$ for omitida, a resposta é afirmativa: basta considerar, sobre o conjunto $X=\omega_{2}+1$, a topologia em que todo $\alpha \in \omega_{2}$ é um ponto isolado e \{]$\left.\left.\alpha, \omega_{2}\right] \mid \alpha \in \omega_{2}\right\}$ é um sistema fundamental de vizinhanças abertas para $\omega_{2} \in X$. Nestas condições, decorre da regularidade de $\omega_{2}$ que todo $Y \in[X]^{\leq \omega_{1}}$ é discreto e, portanto, metrizável; no entanto, $X$ não é metrizável, uma vez que, novamente por ser $\omega_{2}$ um cardinal regular, $\chi\left(\omega_{2}, X\right)=\omega_{2}$.

\footnotetext{
${ }^{1}$ A pergunta, originalmente, foi feita sob a hipótese de $X$ ser normal ao invés de $T_{2}$. No entanto, como não se encontraram exemplos em ZFC de tais espaços mesmo sob a hipótese de $X$ ser $T_{2}$, e como não se obteve resposta negativa sob a hipótese original, hoje a pergunta é considerada com o enunciado aqui apresentado.
} 
O problema de Hamburger permanece sem solução em ZFC; o que se tem até o momento são resultados parciais - mais precisamente, são resultados de consistência ou que assumem hipóteses topológicas adicionais —, como os que veremos a seguir.

Os primeiros teoremas desta seção decorrem do seguinte resultado, obtido implicitamente da demonstração do teorema 3.1 em [5] e enunciado em [32]: ${ }^{2}$

Proposição 3.1.1. Seja $(X, \tau)$ um espaço topológico tal que todo $Y \in[X] \leq d(X)$ é regular $e$ tem caráter enumerável. Então

(i) $(X, \tau)$ é regular;

(ii) $(X, \tau)$ tem caráter enumerável;

(iii) $|X| \leq d(X)^{\omega}$

Demonstração. Fixe $D \in[X] \leq d(X)$ denso em $(X, \tau)$ arbitrário.

Comecemos pelo item $(i)$. Temos que $(X, \tau)$ é $T_{1}$, pois, se houvesse $x \in X$ tal que $\overline{\{x\}} \neq$ $\{x\}$, teríamos que, tomando $y \in \overline{\{x\}} \backslash\{x\}$ qualquer, o subespaço $\{x, y\} \subseteq X$ não seria $T_{1}$, contradizendo a hipótese do teorema.

Para provar que $(X, \tau)$ é $T_{3}$, suponha, por absurdo, que existam $x \in X$ e $U \in \tau_{x}$ tais que $\bar{V} \nsubseteq U$ para todo $V \in \tau_{x}$. Tome agora $M \prec H_{\theta} \operatorname{com}\{X, \tau, D, x, U\} \cup D \subseteq M \mathrm{e}$ $|M| \leq|D| \cdot \omega=d(X)$. Por hipótese, $X \cap M$ é regular, logo existe $V \in \tau_{x}$ tal que $\overline{V \cap M}^{X \cap M} \subseteq$ $U \cap M$; como $X \cap M$ é denso em $X$ - uma vez que $D \subseteq X \cap M$-, temos que $\bar{V} \cap M=$ $\overline{V \cap M} \cap M=\overline{V \cap M}^{X \cap M} \subseteq U \cap M$. Novamente por hipótese, $\chi(D \cup\{x\})=\omega$, de modo que existe $\mathcal{V} \subseteq \tau$ enumerável que induz um sistema fundamental de vizinhanças para $x$ em $D \cup\{x\}$; pelo critério de Tarski, podemos tomar um tal $\mathcal{V} \in M$. Existe então $W \in \mathcal{V}$ tal que $x \in W \cap(D \cup\{x\}) \subseteq V \cap(D \cup\{x\})$; temos que $W \in M$, pois, pelo corolário 1.1.5,

\footnotetext{
${ }^{2}$ Enunciamos aqui esta proposição numa forma um pouco mais geral: suas hipóteses em $[32]$ são " $d(X) \leq \omega_{1}$ e todo $Y \in[X] \leq \omega_{1}$ é metrizável"; a demonstração, no entanto, é essencialmente a mesma.
} 
$\mathcal{V} \subseteq M$. Como $D \cup\{x\}$ é denso em $X$, então $\bar{W}=\overline{W \cap(D \cup\{x\})} \subseteq \overline{V \cap(D \cup\{x\})}=\bar{V}$, logo $\bar{W} \cap M \subseteq \bar{V} \cap M \subseteq U \cap M$, i.e., $M \models \bar{W} \subseteq U$, o que é um absurdo, pois $H_{\theta} \models \bar{W} \backslash U \neq \emptyset \mathrm{e}$ $M \prec H_{\theta}$. Portanto, $(X, \tau)$ é regular.

Agora, para $x \in X$ arbitrário, tem-se que $\chi(x, D \cup\{x\})=\omega$ - por hipótese -; como, pelo item anterior, $(X, \tau) \in \mathcal{T}_{3}$, segue então do lema 1.2.14 que $\chi(x, X)=\omega$, o que demonstra o item $(i i)$.

Finalmente, como $D$ é denso em $X$ e $\chi(X)=\omega$, segue que, para todo $x \in X$, existe uma seqüência $\left(a_{n}^{x}\right)_{n \in \omega}$ em $D$ que converge para $x$. Tem-se então que a função $f: X \rightarrow{ }^{\omega} D$ dada por $f(x)=\left(a_{n}^{x}\right)_{n \in \omega}$ para todo $x \in X$ é injetora - uma vez que $(X, \tau)$ é $T_{2}$-, logo $|X| \leq|\omega|=|D|^{\omega} \leq d(X)^{\omega}$, provando o item (iii). q.e.d.

Cabe notar que, com uma demonstração análoga, é possível obter a seguinte versão - mais geral - desta proposição: se um espaço topológico $(X, \tau)$ e um cardinal infinito $\kappa \leq d(X)$ são tais que todo $Y \in[X]^{\leq d(X)}$ é regular e satisfaz $\chi(Y) \leq \kappa$, então $(X, \tau)$ é regular, $\chi(X) \leq \kappa$ e $|X| \leq d(X)^{\kappa}$.

Veremos agora algumas conseqüências desta proposição, demonstradas em [4] — vide também [32].

A primeira delas é a não-existência de um espaço separável nas condições do problema de Hamburger. Mas, antes, façamos uma pequena observação:

Lema 3.1.2. Se $(X, \tau) \in \mathcal{T}$ admite uma base pontualmente enumerável, então $d(X)=w(X)$.

Demonstração. Já sabemos que $d(X) \leq w(X)$. Sejam agora $\mathcal{B} \subseteq \tau$ uma base pontualmente enumerável para $(X, \tau)$ e $D \in[X]^{\leq d(X)}$ denso em $(X, \tau)$. Tem-se então que $\mathcal{B}=\bigcup_{y \in D}\{U \in$ $\mathcal{B} \mid y \in U\}$, de modo que $|\mathcal{B}| \leq \omega \cdot|D| \leq \omega \cdot d(X)=d(X)$, logo $w(X) \leq|\mathcal{B}|+\omega \leq d(X)+\omega=$ $d(X)$.

q.e.d. 
Corolário 3.1.3. Seja $(X, \tau)$ um espaço topológico separável tal que todo $Y \in[X]^{\leq \omega_{1}}$ é regular e admite uma base pontualmente enumerável ${ }^{3}$. Então $(X, \tau)$ é metrizável.

Demonstração. Seja $D \subseteq X$ enumerável e denso em $(X, \tau)$. Dado $Y \in[X] \leq \omega_{1}$ arbitrário, tem-se, por hipótese, que $Y \cup D$ é regular e admite uma base pontualmente enumerável; como $D$ é denso em $X$, então é denso em $Y \cup D, \operatorname{logo} Y \cup D$ é separável. Pelo lema anterior, tem-se assim que $w(Y \cup D)=\omega$, e portanto $w(Y)=\omega$ - uma vez que $w$ é uma função cardinal monótona. Como $Y$ foi tomado de maneira arbitrária, segue então do teorema 2.2.20 que $w(X)=\omega$. Finalmente, tem-se da proposição 3.1.1 que $(X, \tau)$ é regular, o que implica que $(X, \tau)$ é metrizável em virtude do teorema de Nagata-Smirnov.

q.e.d.

Corolário 3.1.4. Seja $(X, \tau)$ um espaço topológico separável tal que todo $Y \in[X] \leq \omega_{1}$ é metrizável. Então $(X, \tau)$ é metrizável.

Note que, se $(X, \tau)$ é um espaço topológico tal que $d(X)=\omega_{1}$ e todo $Y \in[X]^{\leq \omega_{1}}$ é metrizável, segue da proposição 3.1.1(iii) que $|X| \leq \omega_{1}^{\omega} \leq\left(2^{\omega}\right)^{\omega}=2^{\omega}$. Portanto, assumindose $\mathrm{CH}$, tem-se que $(X, \tau)$ é metrizável — uma vez que, nestas condições, $X \in[X] \leq \omega_{1}$.

Por outro lado, assumindo-se MA $+\neg \mathrm{CH}$, é possível obter um espaço de Lindelöf $(X, \tau)$ regular e não-metrizável tal que $\chi(X)=\omega, d(X)=\omega_{1}$ e todo $Y \in[X]^{\leq \omega_{1}}$ é metrizável vide [4]. Assim sendo, a afirmação que se obtém substituindo-se $d(X)=\omega$ por $d(X) \leq \omega_{1}$ no enunciado do corolário $3.1 .4^{4}$ é independente de ZFC. ${ }^{5}$

O próximo corolário atesta que não existe um espaço nas condições do problema de Hamburger que tenha spread enumerável; para demonstrar tal afirmação, utilizaremos a seguinte propriedade de espaços metrizáveis:

\footnotetext{
${ }^{3}$ Esta afirmação foi originalmente enunciada como no corolário 3.1.4; no entanto, a demonstração apresentada para a mesma permanece válida com estas hipóteses.

${ }^{4}$ Pode-se mesmo incluir as hipóteses de $(X, \tau)$ ser um espaço de Lindelöf, regular e de caráter enumerável.

${ }^{5} \mathrm{~A}$ afirmação, no entanto, torna-se verdadeira ao substituirmos a tese pela afirmação mais fraca " $(X, \tau)$ admite uma base pontualmente enumerável", como veremos mais adiante no teorema 3.3.2.
} 
Proposição 3.1.5. Seja $(X, \tau)$ um espaço topológico metrizável. Então $c(X)=d(X)$.

Demonstração. Como já observamos na proposição 1.2.12(ii), tem-se que $c(X) \leq d(X)$; provemos então que $d(X) \leq c(X)$.

Sejam $\kappa=c(X)$ e $m: X \times X \rightarrow \mathbb{R}_{+}$uma métrica compatível com $\tau$. Para cada $n \in \omega$, seja $\mathscr{C}_{n} \subseteq\left\{B_{m}\left(x, \frac{1}{2^{n}}\right) \mid x \in X\right\}$ uma família celular maximal com respeito à ordem da inclusão ${ }^{6}$. Considere agora $D=\bigcup_{n \in \omega}\left\{x \in X \mid B_{m}\left(x, \frac{1}{2^{n}}\right) \in \mathscr{C}_{n}\right\}$; como $\left|\mathscr{C}_{n}\right| \leq \kappa$ para todo $n \in \omega$, então $|D| \leq \kappa \cdot \omega=\kappa$.

Afirmamos que $D$ é denso em $(X, \tau)$ - o que conclui a demonstração, pois então $d(X) \leq$ $|D|+\omega \leq \kappa+\omega=\kappa=c(X)$. De fato, tome $U \in \tau \backslash\{\emptyset\}$ arbitrário. Existem então $z \in U$ e $p \in \omega$ tais que $B_{m}\left(z, \frac{1}{2^{p}}\right) \subseteq U$. Agora, como $\mathscr{C}_{p+1}$ é maximal, existe $x \in X$ tal que $B_{m}\left(x, \frac{1}{2^{p+1}}\right) \in \mathscr{C}_{p+1}$ e $B_{m}\left(x, \frac{1}{2^{p+1}}\right) \cap B_{m}\left(z, \frac{1}{2^{p+1}}\right) \neq \emptyset$ - pois, do contrário, a família celular $\mathscr{C}_{p+1} \cup\left\{B_{m}\left(z, \frac{1}{2^{p+1}}\right)\right\}$ contradiria a maximalidade de $\mathscr{C}_{p+1}$. Segue então da desigualdade triangular que $m(x, z) \leq \frac{1}{2^{p+1}}+\frac{1}{2^{p+1}}=\frac{1}{2^{p}}, \log x \in B_{m}\left(z, \frac{1}{p}\right) \subseteq U$ e, como $x \in D$, tem-se que $U \cap D \neq \emptyset$.

q.e.d.

Corolário 3.1.6. Seja $(X, \tau)$ um espaço topológico tal que $s(X)=\omega$ e todo $Y \in[X]^{\leq \omega_{1}}$ é metrizável. Então $(X, \tau)$ é metrizável.

Demonstração. Suponha, por absurdo, que $(X, \tau)$ não seja metrizável. Então, pelo corolário 3.1.4, $d(X) \geq \omega_{1}$, e segue assim da proposição 2.2 .3 que existe $S \in[X]^{\omega_{1}}$ separado à esquerda. Nestas condições, $d(S)=\omega_{1}$ e, como $S$ é, por hipótese, metrizável, tem-se da proposição anterior que $c(S)=\omega_{1}$, o que implica que $s(X)=h c(X) \geq c(S)=\omega_{1}$, uma contradição.

q.e.d.

O próximo teorema — obtido em [5] — garante que não existe um espaço nas condições do problema de Hamburger que seja enumeravelmente compacto. Mas, antes, provemos uma afirmação da qual faremos uso algumas vezes neste capítulo:

\footnotetext{
${ }^{6}$ Uma tal família pode ser facilmente obtida fazendo-se uso do lema de Kuratowski-Zorn.
} 
Lema 3.1.7. Sejam $(X, \tau)$ um espaço topológico e $M \prec H_{\theta}$ tal que $\chi(X) \cup\{X, \tau, \chi(X)\} \subseteq M$. Então $\tau \cap M$ contém um sistema fundamental de vizinhanças em $(X, \tau)$ para cada ponto de $X \cap M$.

Demonstração. Tome $x \in X \cap M$ arbitrário. Por hipótese, existe $\mathcal{V} \subseteq \tau$ tal que $|\mathcal{V}| \leq \chi(X)$ e que constitui um sistema fundamental de vizinhanças para $x$ em $(X, \tau)$; logo, pelo critério de Tarski, existe um tal $\mathcal{V} \in M$. Segue então do corolário 1.1.4 que $\mathcal{V} \subseteq \tau \cap M$. q.e.d.

Teorema 3.1.8. Seja $(X, \tau)$ um espaço de Hausdorff enumeravelmente compacto tal que todo $Y \in[X]^{\leq \omega_{1}}$ é regular e admite uma base pontualmente enumerável ${ }^{T}$. Então $(X, \tau)$ é metrizável.

Demonstração. Pelo corolário 1.2.11, é suficiente mostrar que $w(X)=\omega$. Suponha, por absurdo, que isso não ocorra.

Seja $\left(M_{\alpha}\right)_{\alpha \in \omega_{1}}$ como na demonstração do lema 1.1.12, tomando $\kappa=\omega$ e $A=\left\{X, \tau, \omega_{1}\right\}$. Como $w(X) \geq \omega_{1}$, segue do teorema 2.2.20 que existe $Y \in[X]^{\leq \omega_{1}}$ tal que $w(Y) \geq \omega_{1}$; $\log$, pelo critério de Tarski, existe um tal $Y \in M=\bigcup_{\alpha \in \omega_{1}} M_{\alpha} \prec H_{\theta}$. Considere agora $Z=\bar{Y} \in M$, e note que $w(Z) \geq w(Y) \geq \omega_{1}$.

Deste ponto em diante, consideraremos apenas o espaço topológico $\left(Z, \tau^{Z}\right) \in M$; em particular, designaremos a aderência em $\left(Z, \tau^{Z}\right)$ de um conjunto $E \subseteq Z$ simplesmente por $\bar{E}$.

Podemos supor, sem perda de generalidade, que $\left\{Z, \tau^{Z}\right\} \subseteq M_{1}$, pois $\left\{Z, \tau^{Z}\right\} \subseteq M_{\xi}$ para todo $\xi \in \omega_{1}$ maior que um certo $\xi_{0} \in \omega_{1}$. Temos, para todo $\alpha \in \omega_{1} \backslash\{0\}$, que $\left|M_{\alpha}\right|=\omega$, $\operatorname{logo} \tau^{Z} \cap M_{\alpha}$ não é uma base para $Z$ - pois, como já observamos, $w(Z) \geq \omega_{1}$. Assim, pelo lema 1.2.9, existe $x_{\alpha} \in \overline{Z \cap M_{\alpha}}$ tal que $\tau^{Z} \cap M_{\alpha}$ não contém um sistema fundamental de vizinhanças para $x_{\alpha}$ em $Z$; pelo critério de Tarski, podemos tomar um tal $x_{\alpha} \in M_{\alpha+1}$, uma vez que $M_{\alpha} \in M_{\alpha+1}$.

Como $Y$ é denso em $\left(Z, \tau^{Z}\right)$, então $d(Z) \leq \omega_{1}$; logo, pela proposição $3.1 .1,\left(Z, \tau^{Z}\right)$ é regular

\footnotetext{
${ }^{7}$ Assim como fizemos em 3.1.3, enfraquecemos a hipótese deste teorema — que foi enunciado em [5] como o enunciamos em 3.1.9 - devido ao fato de que sua demonstração original aplica-se igualmente para este enunciado.
} 
e tem caráter enumerável. Assim, pelo lema 3.1.7, $\tau^{Z} \cap M$ induz uma base de abertos em $Z \cap M$; consideremos então a base de abertos $\mathcal{B}_{M}=\left\{U \cap(Z \cap M) \mid U \in \tau^{Z} \cap M\right\}$ para $Z \cap M$.

Tome agora $N \prec H_{\theta}$ enumerável tal que $\left\{Z, \tau^{Z},\left(M_{\alpha}\right)_{\alpha \in \omega_{1}}\right\} \subseteq N$, e seja $\gamma=N \cap \omega_{1}$ - que, pelo lema 1.1.13, é um ordinal limite —; como $N$ é enumerável, segue que $\gamma \in \omega_{1}$. Pelo lema 1.1.14(b), temos que $M \cap N=M_{\gamma}$, $\operatorname{logo} \tau^{Z} \cap M \cap N=\tau^{Z} \cap M_{\gamma}$. Assim, pela escolha de $x_{\gamma}$, temse que $\tau^{Z} \cap M \cap N$ não induz - e, portanto, $\mathcal{B}_{M} \cap N$ não contém — um sistema fundamental de vizinhanças para $x_{\gamma} \in \overline{Z \cap M_{\gamma}} \cap(Z \cap M)=\overline{(Z \cap M) \cap N} \cap(Z \cap M)=\overline{(Z \cap M) \cap N}^{Z \cap M}$ em $Z \cap M$, e segue então do lema 1.2.8 que $Z \cap M$ não admite base pontualmente enumerável, contrariando a hipótese do teorema. q.e.d.

Corolário 3.1.9. Seja $(X, \tau)$ um espaço de Hausdorff enumeravelmente compacto tal que todo $Y \in[X] \leq \omega_{1}$ é metrizável. Então $(X, \tau)$ é metrizável.

Cabe citar que o teorema 3.1.8 será obtido mais adiante - corolário 3.3.3 — sob uma hipótese mais fraca - a saber, $(X, \tau) \in \mathcal{T}_{1}$ ao invés de $(X, \tau) \in \mathcal{T}_{2}$. A opção de incluir sua demonstração original deveu-se ao fato de que a mesma apresenta interesse próprio, ilustrando — de modo mais simples que na seção 3.3 - a técnica de se tomar um $N \prec H_{\theta}$ ao qual pertença uma cadeia elementar de $H_{\theta}$ previamente considerada.

Veremos agora que é consistente com ZFC — mais especificamente, decorre de $V=L$ que o problema de Hamburger tenha resposta afirmativa.

O resultado crucial que nos permitirá obter um exemplo de um espaço nas condições requeridas é:

Teorema 3.1.10. Assuma $V=L$. Então existe $E \subseteq\left\{\alpha \in \omega_{2} \mid c f(\alpha)=\omega\right\}$ tal que E é estacionário em $\omega_{2}$ e, para todo $\beta \in \lim \left(\omega_{2}\right)$, o conjunto $E \cap \beta$ não é estacionário em $\beta$.

Demonstração. Pelo teorema 1.1.1, existe $E \subseteq\left\{\alpha \in \omega_{2} \mid \operatorname{cf}(\alpha)=\omega\right\}$ estacionário em $\omega_{2}$ satisfazendo $\square_{\omega_{1}}(E)$. Fixe então uma $\square_{\omega_{1}}(E)$-seqüência $\left(C_{\alpha}\right)_{\alpha \in \lim \left(\omega_{2}\right)}$ e tome $\beta \in \lim \left(\omega_{2}\right)$ arbitrário. Se $c f(\beta)=\omega$, considere $S \subseteq \beta$ cofinal em $\beta$ tal que $t p(S)=\omega$ e todo elemento de 
$S$ é um ordinal sucessor; nestas condições, tem-se que $S$ é c.u.b. em $\beta$ e é disjunto de $E \cap \beta$, de modo que $E \cap \beta$ não é estacionário em $\beta$. Já se $c f(\beta)=\omega_{1}$, temos que $\lim \left(C_{\beta}\right)$ é c.u.b. em $\beta$; assim, como $(E \cap \beta) \cap \lim \left(C_{\beta}\right)=\emptyset$ - por (iii) em $\square_{\omega_{1}}(E)$-, segue que $E \cap \beta$ não é estacionário em $\beta$.

q.e.d.

Um conjunto $E$ nas condições do teorema anterior recebe o nome de $E$-set.

A partir desse resultado, o primeiro exemplo de um espaço que verifica as condições do problema de Hamburger — retirado de [15] — é bastante direto:

Teorema 3.1.11. Assuma $V=L$ e tome um E-set E. Então E, com a topologia de subespaço herdada de $\omega_{2}$, é um espaço não-metrizável e de caráter enumerável tal que todo $Y \in[E]^{\leq \omega_{1}}$ é metrizável.

Mas, antes, precisaremos de um lema:

Lema 3.1.12. Seja $X$ um espaço topológico regular de caráter enumerável. Seja ainda $p \in X$ tal que $X \backslash\{p\}$ é metrizável. Então $X$ é metrizável.

Demonstração. Sejam $\left\{V_{n} \mid n \in \omega\right\}$ um sistema fundamental de vizinhanças para $p$ em $X$ e $\mathcal{B}=\bigcup_{m \in \omega} \mathcal{B}_{m}$ uma base $\sigma$-localmente finita de $X \backslash\{p\}$, com $\mathcal{B}_{m}$ localmente finito para todo $m \in \omega$ - a existência de uma tal base é garantida pelo teorema de Nagata-Smirnov.

Defina, para todo $(m, n) \in \omega \times \omega$,

$$
\mathcal{B}_{(m, n)}^{\prime}=\left\{B \backslash \overline{V_{n}} \mid B \in \mathcal{B}_{m}\right\} \cup\left\{V_{n}\right\},
$$

e $\mathcal{B}^{\prime}=\bigcup\left\{\mathcal{B}_{(m, n)}^{\prime} \mid(m, n) \in \omega \times \omega\right\}$ será uma base $\sigma$-localmente finita de $X$.

q.e.d.

Demonstração do teorema 3.1.11. O fato de que $E$ tem caráter enumerável decorre imediatamente do fato de que $c f(\alpha)=\omega$ para todo $\alpha \in E$ : dados $\alpha \in E$ e $\left\{\alpha_{n} \mid n \in \omega\right\} \subseteq \alpha$ cofinal em $\alpha$, tem-se que o conjunto \{]$\left.\left.\alpha_{n}, \alpha\right] \cap E \mid n \in \omega\right\}$ é um sistema fundamental de vizinhanças para $\alpha$ em $E$. 
Suponha agora, por absurdo, que $E$ seja metrizável; pelo teorema de Nagata-Smirnov, temos então que $E$, em particular, admite uma base pontualmente enumerável - digamos, $\mathcal{B}$. Para cada $\alpha \in E$, tome $U_{\alpha} \in \mathcal{B}$ tal que $\alpha \in U_{\alpha} \subseteq[0, \alpha]$. Temos então, pela definição da topologia da ordem, que existe uma função $f: E \rightarrow \omega_{2}$ tal que, para todo $\alpha \in E$, valem $f(\alpha)<\alpha$ e $] f(\alpha), \alpha] \cap E \subseteq U_{\alpha}$. Assim, pelo lema pressing-down, existe $\gamma \in \omega_{2}$ tal que $F=\{\alpha \in E \mid f(\alpha)=\gamma\} \subseteq \omega_{2}$ é estacionário em $\omega_{2}$. Tomando então $\delta=\min (E \cap] \gamma, \omega_{2}[)$, temos que $\delta \in] f(\beta), \beta]$ para todo $\beta \in F$, de modo que $\delta \in U_{\beta}$ para todo $\beta \in F$; isto contradiz a hipótese de que $\mathcal{B}$ é pontualmente enumerável, pois a função $\alpha \in E \mapsto U_{\alpha} \in \mathcal{B}$ é injetora e $|F|=\omega_{2}$.

Finalmente, provemos que todo $Y \in[E]^{\leq \omega_{1}}$ é metrizável. Procederemos por indução transfinita em $\lambda=t p(Y)$.

Tome $Y \in[E]^{\leq \omega_{1}}$ arbitrário e suponha que a afirmação seja válida para todo $Z \in[E]^{\leq \omega_{1}}$ com $t p(Z)<\lambda=t p(Y)$. Se $\lambda$ é um ordinal sucessor, o resultado segue do lema 3.1.12 - pois, como observamos na primeira seção, todo subconjunto de um ordinal é um espaço topológico regular na topologia da ordem. Suponha então que $\lambda$ é um ordinal limite.

Seja $\sigma=\sup Y$; como $\omega_{2}$ é um cardinal regular e $\lambda$ é um ordinal limite, então $\sigma \in \lim \left(\omega_{2}\right)$, $\operatorname{logo} E \cap \sigma$ não é estacionário em $\sigma$. Assim sendo, $Y \subseteq E \cap \sigma$ também não é estacionário em $\sigma$, de modo que existe $C \subseteq \sigma$ c.u.b. em $\sigma$ tal que $C \cap Y=\emptyset$. Como $\sigma \backslash C$ é aberto em $\sigma$, existe então uma família $\left\{J_{i} \mid i \in \mathcal{I}\right\}$ de intervalos abertos em $\sigma$ satisfazendo as condições do lema 1.2.4 para $\sigma \backslash C$.

Considere $\lambda_{i}=\operatorname{tp}\left(J_{i} \cap Y\right)$ para cada $i \in \mathcal{I}$. Dado $i \in \mathcal{I}$ arbitrário, temos que $\lambda_{i}<\lambda$, pois $C$ é ilimitado em $\sigma$; assim, pela hipótese de indução, $J_{i} \cap Y$ é metrizável. A metrizabilidade de $Y$ decorre então do corolário 1.2.7 - uma vez que $Y=\dot{\bigcup}_{i \in \mathcal{I}}\left(J_{i} \cap Y\right)$ e todo $J_{i} \cap Y$ é aberto em $Y$.

q.e.d.

Cabe observar que, como $E$ é um subespaço de $\omega_{2}$, então $E$ é hereditariamente normal. 


\subsection{Espaços $\omega_{1}$-coletivamente de Hausdorff}

Trataremos agora de uma outra pergunta, feita ${ }^{8}$ em [9]:

Existe algum espaço $\omega_{1}$-coletivamente de Hausdorff de caráter enumerável que não é coletivamente de Hausdorff?

Omitindo-se a hipótese do caráter enumerável, a pergunta apresentada tem resposta afirmativa - vide, e.g., [27]. Considerando-se tal hipótese, no entanto, a pergunta permanece sem resposta em ZFC até o momento — vide, e.g., [29].

Embora esta pergunta não se trate exatamente de uma propriedade de reflexão — no sentido considerado nesta dissertação - ela se relaciona a uma tal propriedade; mais precisamente, tem-se:

Proposição 3.2.1. Se existe um espaço $\omega_{1}$-coletivamente de Hausdorff de caráter enumerável que não é coletivamente de Hausdorff, então existe um espaço de Hausdorff $(X, \tau)$ de caráter enumerável que não é coletivamente de Hausdorff e tal que todo $Y \in[X] \leq \omega_{1}$ é coletivamente de Hausdorff.

Demonstração. Seja $\left(Z, \tau^{\prime}\right)$ um espaço topológico nas condições da hipótese; existe então $D \subseteq$ $Z$ discreto e fechado em $\left(Z, \tau^{\prime}\right)$ que testemunha o fato de que $\left(Z, \tau^{\prime}\right)$ não é coletivamente de Hausdorff. Considere, sobre $X=Z$, a topologia $\tau$ em que $\{x\}$ é aberto para todo $x \in X \backslash D$ e, para todo $y \in D$, as vizinhanças de $y$ em $(X, \tau)$ são exatamente as vizinhanças de $y$ em $\left(Z, \tau^{\prime}\right)$.

Como $\tau^{\prime}$ é $T_{2}$ e $\tau^{\prime} \subseteq \tau$, então $\tau$ também é $T_{2}$; além disso, $\chi(X)=\omega$, uma vez que todo $x \in X \backslash D$ é ponto isolado de $(X, \tau)$ e $\chi(y, X)=\chi(y, Z)=\omega$ para todo $y \in D$. Tem-se ainda que $(X, \tau)$ não é coletivamente de Hausdorff, pois $D \subseteq X$ é discreto e fechado em $(X, \tau)$ — já que o era em $\left(Z, \tau^{\prime}\right)$ e $\tau^{\prime} \subseteq \tau$ - e as vizinhanças de seus pontos não foram alteradas.

\footnotetext{
${ }^{8}$ Com a hipótese de o espaço ser normal ao invés de $T_{2}$.
} 
Finalmente, tome $Y \in[X]^{\leq \omega_{1}}$ arbitrário e $F \subseteq Y$ discreto e fechado em $(X, \tau)$. Note que, como $D$ não admite ponto de acumulação em $\left(Z, \tau^{\prime}\right)$ - por ser discreto e fechado -, o mesmo ocorre com qualquer subconjunto seu; assim, $F \cap D$ é discreto e fechado em $\left(Z, \tau^{\prime}\right)$. Segue então da hipótese que existe uma família celular $\left\{U_{y} \mid y \in F \cap D\right\} \subseteq \tau^{\prime} \subseteq \tau$ satisfazendo $y \in U_{y}$ para todo $y \in F \cap D$. Agora, como $F$ não admite ponto de acumulação em $(X, \tau)$, tem-se que, para cada $y \in F \cap D$, existe $V_{y} \in \tau$ com $V_{y} \cap F=\{y\}$. Nestas condições, definido-se $W_{y}=U_{y} \cap V_{y}$ para todo $y \in F \cap D$ e $W_{x}=\{x\}$ para todo $x \in F \backslash D$, tem-se que $\left\{W_{x} \mid x \in F\right\}$ é uma família celular em $(X, \tau)$ tal que $x \in W_{x}$ para todo $x \in F-\mathrm{e}$, assim, $\left\{W_{x} \cap Y \mid x \in F\right\}$ é a família celular no subespaço $Y \subseteq X$ procurada.

q.e.d.

Em princípio, a reflexão da propriedade de o espaço ser coletivamente de Hausdorff — para espaços de Hausdorff de caráter enumerável - é mais pertinente ao tema desta dissertação que a pergunta apresentada; no entanto, esta última é um problema mais clássico e ocorre com maior freqüência na literatura ${ }^{9}$ — vide, e.g., [29] —, o que justifica sua presença neste trabalho sob esta forma ${ }^{10}$. Além disso, surge também uma interessante relação entre a mesma e o problema de Hamburger, como se vê pelo resultado a seguir ${ }^{11}$ - e.g., 4.4 em [29] —:

Proposição 3.2.2. Se existe um espaço $\omega_{1}$-coletivamente de Hausdorff de caráter enumerável que não é coletivamente de Hausdorff, então existe um espaço de Hausdorff $X$ não-metrizável e de caráter enumerável tal que todo $Y \in[X]^{\leq \omega_{1}}$ é metrizável.

Demonstração. Suponha que $\left(Z, \tau^{\prime}\right)$ seja um espaço nas condições da hipótese, e fixe $D \subseteq Z$ discreto e fechado em $\left(Z, \tau^{\prime}\right)$ que testemunha o fato de que $\left(Z, \tau^{\prime}\right)$ não é coletivamente de Hausdorff. Considere, no conjunto $X=Z$, a topologia $\tau$ gerada por $\tau^{\prime} \cup\{\{z\} \mid z \in X \backslash D\}$. Como $\left(Z, \tau^{\prime}\right)$ é $T_{2}$ e tem caráter enumerável, o mesmo ocorre com $(X, \tau)$; além disso, $(X, \tau)$

\footnotetext{
${ }^{9}$ Muitas vezes, substituindo-se "coletivamente de Hausdorff" por " $\lambda$-coletivamente de Hausdorff" e " $\omega_{1}$ coletivamente de Hausdorff" por " $\kappa$-coletivamente de Hausdorff para todo cardinal $\kappa<\lambda$ ", sendo $\kappa$ um cardinal maior ou igual a $\omega_{2}$ - vide, e.g., [9] e [30].

${ }^{10}$ Cabe citar que a pergunta de reflexão também é estudada; vide, e.g., [11].

${ }^{11}$ Cuja demonstração se assemelha à da proposição anterior.
} 
não é coletivamente de Hausdorff — logo, pela proposição 1.2.3, não é metrizável —, pois o mesmo $D \subseteq X$ testemunha este fato.

Tome agora $Y \in[X]^{\leq \omega_{1}}$ arbitrário. Como $Y \cap D \in[Z]^{\leq \omega_{1}}$ é discreto e fechado em $\left(Z, \tau^{\prime}\right)$, existe uma família celular $\left\{U_{y} \mid y \in Y \cap D\right\} \subseteq \tau^{\prime} \subseteq \tau$ tal que $y \in U_{y}$ para todo $y \in Y \cap D$. Pela lema 3.1.12, cada $U_{y} \subseteq X$ é metrizável como subespaço de $(X, \tau)$; assim, pelo corolário 1.2.7,

$$
\bigcup_{y \in Y \cap D} U_{y} \subseteq X
$$

também o é. Portanto, novamente pelo corolário 1.2.7, o mesmo ocorre com

$$
Y=\left(Y \cap\left(\bigcup_{y \in Y \cap D} U_{y}\right)\right) \cup\left(Y \backslash\left(\bigcup_{y \in Y \cap D} U_{y}\right)\right) \subseteq X
$$

q.e.d.

Vamos agora, novamente a partir de 3.1.10, construir um exemplo a partir de $V=L-$ retirado de [10] — de um espaço $\omega_{1}$-coletivamente de Hausdorff de caráter enumerável que não é coletivamente de Hausdorff. ${ }^{12}$

Assuma $V=L$ e tome um E-set $E$. Para cada $\alpha \in E$, fixe uma seqüência estritamente crescente $s_{\alpha}: \omega \rightarrow \alpha$ tal que $s_{\alpha}(n)$ é um ordinal sucessor para todo $n \in \omega$ e $\sup \left(s_{\alpha}[\omega]\right)=\alpha$. Seja agora $D=\left\{s_{\alpha}(n) \mid \alpha \in E\right.$ e $\left.n \in \omega\right\}$ — note que $D \cap E=\emptyset$ - e defina

$$
V_{\alpha}^{n}=\{\alpha\} \cup\left\{s_{\alpha}(m) \mid m \in \omega \text { e } m \geq n\right\}
$$

para cada $\alpha \in E$ e cada $n \in \omega$.

Considere então, sobre o conjunto $S=E \cup D$, a topologia em que:

\footnotetext{
${ }^{12}$ Cabe ainda comentar que tal exemplo se trata de um espaço de Moore - embora tal conceito não tenha sido trabalhado neste texto, ressaltamos este fato para interesse do leitor, uma vez que o estudo de espaços $\kappa$-coletivamente de Hausdorff teve origem no estudo da metrizabilidade de espaços de Moore; vide, e.g., [9] e [10].
} 
- todo elemento de $D$ é um ponto isolado de $S$;

- para todo $\alpha \in E$, o conjunto $\left\{V_{\alpha}^{n} \mid n \in \omega\right\}$ é um sistema fundamental de vizinhanças para $\alpha$ em $S$.

Teorema 3.2.3. O espaço topológico $S$ definido na página anterior é $\omega_{1}$-coletivamente de Hausdorff, possui caráter enumerável e não é coletivamente de Hausdorff.

Demonstração. É imediato que $S$ tem caráter enumerável.

Suponha, por absurdo, que $S$ seja coletivamente de Hausdorff. Temos que $E \subseteq S$ é fechado e discreto; assim, para cada $\alpha \in E$ deve existir $n_{\alpha} \in \omega$ de modo a $\left\{V_{\alpha}^{n_{\alpha}} \mid \alpha \in E\right\}$ ser uma família celular. Defina agora

$$
\begin{aligned}
f: E & \rightarrow \omega_{2} \\
\alpha & \mapsto s_{\alpha}\left(n_{\alpha}\right),
\end{aligned}
$$

que é uma função injetora, uma vez que $\left\{V_{\alpha}^{n_{\alpha}} \mid \alpha \in E\right\}$ é uma família celular. Temos então um absurdo, pois, aplicando o lema pressing-down, obtemos $\gamma \in \omega_{2}$ tal que $F=\left\{\alpha \in E \mid s_{\alpha}\left(n_{\alpha}\right)=\right.$ $\gamma\} \subseteq \omega_{2}$ é estacionário em $\omega_{2}$.

Finalmente, provemos que $S$ é $\omega_{1}$-coletivamente de Hausdorff. Seja $Y \in[S]^{\leq \omega_{1}}$ fechado e discreto; como $Y$ não admite ponto de acumulação em $S$, temos que $\left\{m \in \omega \mid s_{\alpha}(m) \in Y\right\}$ é finito para todo $\alpha \in E$. Assim, todo $\alpha \in Y \cap E$ admite uma vizinhança disjunta de $Y \cap D=Y \backslash(Y \cap E)$; como os pontos de $Y \cap D$ são isolados, podemos então supor que $Y \subseteq E$.

Procederemos novamente por indução transfinita em $\lambda=t p(Y)$. Suponha que todo $Z \in[E]^{\leq \omega_{1}}$ discreto e fechado em $S$ tal que $t p(Z)<\lambda=t p(Y)$ admita uma família celular $\left\{U_{\alpha}^{Z} \mid \alpha \in Z\right\}$ em $S$ tal que $\alpha \in U_{\alpha}^{Z}$ para todo $\alpha \in Z$. O caso em que $\lambda$ é um ordinal sucessor é imediato, pois, sendo $\xi \in Y$ o último elemento de $Y$ na ordem $\in$, temos que $\xi>\sup (Y \backslash\{\xi\})$; logo, como $\xi \in Y \subseteq E$ é um ordinal limite, existe $n \in \omega$ tal que $s_{\xi}(n)>\sup (Y \backslash\{\xi\})$ e, nestas condições, $\left\{U_{\alpha}^{Y \backslash\{\xi\}} \mid \alpha \in Y \backslash\{\xi\}\right\} \cup\left\{V_{\xi}^{n}\right\}$ é uma família celular.

O caso em que $\lambda$ é um ordinal limite será tratado de maneira similar à feita na demonstração do teorema 3.1.11. Considere, no ordinal $\sigma=\sup Y \in \lim \left(\omega_{2}\right)$, a topologia da ordem. Temos 
que $E \cap \sigma$ não é estacionário em $\sigma$, logo existe $C \subseteq \sigma$ c.u.b. em $\sigma$ tal que $C \cap Y \subseteq C \cap(E \cap \sigma)=$ $\emptyset$. Assim sendo, podemos encontrar uma família $\left\{J_{i} \mid i \in \mathcal{I}\right\}$ de intervalos abertos em $\sigma$ satisfazendo as condições do lema 1.2.4 para o aberto $\sigma \backslash C \subseteq \sigma$. Para cada $\alpha \in Y$, fixe $i_{\alpha} \in \mathcal{I}$ e $n_{\alpha} \in \omega$ tais que $\left.] s_{\alpha}\left(n_{\alpha}\right), \alpha\right] \subseteq J_{i_{\alpha}}$ - isto é sempre possível, pois $\alpha$ é um ordinal limite e todo $J_{i}$ é um intervalo aberto. Aplicando então a hipótese de indução a cada $Y \cap J_{i}-$ uma vez que, como $C \subseteq \sigma$ é ilimitado, então $t p\left(Y \cap J_{i}\right)<\lambda-$, obtemos uma família celular $\left\{W_{\alpha}^{i} \mid \alpha \in Y \cap J_{i}\right\}$ em $S$ tal que $\alpha \in W_{\alpha}^{i}$ para todo $\alpha \in Y \cap J_{i}$. Para cada $\alpha \in Y$, seja agora $m_{\alpha} \in \omega$ tal que $V_{\alpha}^{m_{\alpha}} \subseteq W_{\alpha}^{i_{\alpha}} \cap V_{\alpha}^{n_{\alpha}}$. Temos então, pela escolha de $n_{\alpha}$, que $\left\{V_{\alpha}^{m_{\alpha}} \mid \alpha \in Y\right\}$ é uma família celular em $S$ tal que $\alpha \in V_{\alpha}^{m_{\alpha}}$ para todo $\alpha \in Y$.

q.e.d.

Uma última observação pertinente a este tópico: assumindo-se $\mathfrak{b}>\omega_{1}$ - uma hipótese ${ }^{13}$ da teoria dos conjuntos que é independente de $\mathrm{ZFC}$, inconsistente com $\mathrm{ZFC}+\mathrm{CH}$ e que decorre, por exemplo, de $\mathrm{MA}+\neg \mathrm{CH}-$, é também possível obter um espaço $\omega_{1}$-coletivamente de Hausdorff $X$ de caráter enumerável que não é coletivamente de Hausdorff — vide [7].

\subsection{Reflexão de bases pontualmente enumeráveis}

Nesta seção — baseada em [2] —, trataremos da reflexão com respeito a $\omega_{1}$ da propriedade de o espaço ser regular e admitir uma base pontualmente enumerável ${ }^{14}$. O resultado principal é o teorema 3.3.2, do qual várias conseqüências são obtidas; dentre elas, merece destaque o corolário 3.3.3, que é uma versão mais forte do teorema 3.1.8.

\footnotetext{
${ }^{13}$ Não entraremos em detalhes sobre tal hipótese no presente trabalho; para maiores informações sobre a mesma — bem como sobre outras hipóteses relacionadas —, vide, e.g., [7].

${ }^{14}$ Cabe citar que, embora nosso interesse se concentre em tal caso — o que, em grande parte, se deve ao fato de que buscamos relacionar os resultados desta seção ao problema de Hamburger —, seria possível tratar, obtendo resultados análogos, da reflexão da função cardinal $p w$, definida para todo $X \in \mathcal{T}$ por

$$
p w(X)=\min \left\{\kappa \mid \exists \mathcal{B}\left(\mathcal{B} \text { é uma base de abertos para } X \text { e }\left|\mathcal{B} \cap \tau_{x}\right| \leq \kappa\right)\right\}+\omega
$$
}


O teorema 3.3.2 é uma variação do teorema 1.5 em [2]; o resultado original assume que $(X, \tau) \in \mathcal{T}_{3}$ e, ao invés de supor a existência de uma base pontualmente enumerável para cada $Y \in[X]^{\leq \omega_{1}}$, tal hipótese é restrita apenas a um subconjunto de $[X]^{\leq \omega_{1}}$ - o conjunto dos ditos subespaços thin-short de $(X, \tau)$. Como o conceito de espaço thin-short é um tanto quanto específico e nosso interesse se concentra em resultados de reflexão de propriedades verificadas por subespaços de cardinalidade limitada, tal hipótese foi substituída - com a vantagem de se obter uma demonstração mais simples do resultado em questão, além de se poder suprimir a hipótese de $(X, \tau)$ verificar $T_{3}$, possibilitando que resultados já conhecidos sejam obtidos sem que se faça necessário assumir propriedades de separação adicionais.

Apresentemos então um lema e, em seguida, o teorema.

Lema 3.3.1. Seja $(X, \tau)$ um espaço topológico verificando $t(X)=\omega$ e $d(X) \leq \omega_{1}$. Sejam ainda $M_{0}=\emptyset$ e $\left\{M_{\alpha} \mid 0<\alpha<\omega_{1}\right\}$ uma $\epsilon$-cadeia elementar contínua de $H_{\theta}$ tal que $\left\{X, \tau, \omega_{1}\right\} \subseteq M_{1}$ e, para todo $\alpha \in \omega_{1}, M_{\alpha}$ é enumerável e $\alpha \in M_{\alpha+1}$. Suponha que exista $C \subseteq \omega_{1}$ c.u.b. em $\omega_{1}$ tal que, para todo $\alpha \in C, \tau \cap M_{\alpha}$ contém um sistema fundamental de vizinhanças em $(X, \tau)$ para cada ponto de $\overline{X \cap M_{\alpha}}$. Então $(X, \tau)$ admite uma base pontualmente enumerável.

Demonstração. Podemos, sem perda de generalidade, supor que $C=\omega_{1}$, pois $\operatorname{tp}(C)=\omega_{1}$ e, como $C$ é fechado em $\omega_{1}$, tem-se que $\left\{M_{\alpha} \mid \alpha \in C\right.$ e $\left.0<\alpha\right\}$ é também uma $\epsilon$-cadeia elementar contínua de $H_{\theta}$. Considere, para cada $\alpha \in \omega_{1}, \mathcal{B}_{\alpha}=\left\{U \backslash \overline{X \cap M_{\alpha}} \mid U \in \tau \cap M_{\alpha+1}\right\}$, e defina $\mathcal{B}=\bigcup\left\{\mathcal{B}_{\alpha} \mid \alpha \in \omega_{1}\right\}$.

Afirmamos que $\mathcal{B}$ é pontualmente enumerável. De fato, fixe $x \in X$ arbitrário e seja $M=\bigcup\left\{M_{\alpha} \mid \alpha \in \omega_{1}\right\} \prec H_{\theta}$. Por hipótese, existe $D \in[X]^{\leq \omega_{1}}$ denso em $X$; assim, pelo critério de Tarski, existe um tal $D \in M$ e, nestas condições, segue do lema 1.1.4 que $D \subseteq M$ — pois $\omega_{1} \subseteq M$, uma vez que todo $\alpha \in \omega_{1}$ satisfaz $\alpha \in M_{\alpha+1} \subseteq M$. Assim,

$$
X=\bigcup\left\{\bar{A} \mid A \in[D]^{\leq \omega}\right\} \subseteq \bigcup\left\{\bar{A} \mid A \in[X \cap M]^{\leq \omega}\right\} \subseteq \bigcup_{\alpha \in \omega_{1}} \overline{X \cap M_{\alpha}}
$$


sendo que a primeira igualdade decorre do fato de que $D$ é denso em $X$ e $t(X)=\omega$, e a última inclusão decorre da regularidade de $\omega_{1}$. Portanto, existe $\alpha \in \omega_{1}$ tal que $x \in \overline{X \cap M_{\alpha}}$, de modo que $\{U \in \mathcal{B} \mid x \in U\} \subseteq \bigcup\left\{\mathcal{B}_{\beta} \mid \beta \in \alpha\right\}$, e o resultado segue do fato de que $\alpha$ é enumerável e $\mathcal{B}_{\beta}$ é enumerável para todo $\beta \in \omega_{1}$.

Provemos agora que $\mathcal{B}$ é uma base de abertos para $(X, \tau)$. Para tanto, tome $x \in X$ e $\Omega \in \tau$ arbitrários com $x \in \Omega$, e seja $\alpha=\min \left\{\gamma \in \omega_{1} \mid x \in \overline{X \cap M_{\gamma}}\right\} \geq 1$. Por hipótese, $\tau \cap M_{\alpha}$ contém um sistema fundamental de vizinhanças para $x$ em $(X, \tau)$, logo existe $U \in \tau \cap M_{\alpha}$ tal que $x \in U \subseteq \Omega$. Agora, se $\alpha$ é um ordinal sucessor - digamos, $\alpha=\beta+1$-, então, pela minimalidade de $\alpha$, temos que $V=U \backslash \overline{X \cap M_{\beta}} \in \mathcal{B}_{\beta} \subseteq \mathcal{B}$ verifica $x \in V \subseteq \Omega$. Já se $\alpha$ é um ordinal limite, existe $\beta \in \alpha$ tal que $U \in \tau \cap M_{\beta+1}$, e novamente a minimalidade de $\alpha$ implica que $V=U \backslash \overline{X \cap M_{\beta}} \in \mathcal{B}_{\beta} \subseteq \mathcal{B}$ satisfaz $x \in V \subseteq \Omega$.

q.e.d.

Teorema 3.3.2. Seja $(X, \tau)$ um espaço topológico tal que $d(X) \leq \omega_{1}$ e todo $Y \in[X]^{\leq \omega_{1}}$ é regular e admite uma base pontualmente enumerável. Então $(X, \tau)$ é regular e admite uma base pontualmente enumerável.

Demonstração. O fato de que $(X, \tau)$ é regular decorre da proposição 3.1.1(i).

Se $d(X)=\omega$, o resultado segue do corolário 3.1.3; suponha então que $d(X)=\omega_{1}$, e fixe $D=\left\{a_{\xi} \mid \xi \in \omega_{1}\right\} \in[X]^{\omega_{1}}$ denso em $(X, \tau)$. Construamos, indutivamente, $\left\{M_{\alpha} \mid \alpha \in \omega_{1}\right\}$ nas condições do enunciado do lema 3.3.1.

- Defina $M_{0}=\emptyset$ e tome $M_{1} \prec H_{\theta}$ enumerável tal que $\left\{X, \tau, D, \omega_{1}, a_{0}\right\} \subseteq M_{1}$.

- Em posse de $M_{\beta}$ para um dado $\beta \in \omega_{1}$, tome $M_{\beta+1} \prec H_{\theta}$ enumerável tal que $M_{\beta} \cup$ $\left\{M_{\beta}, a_{\beta}\right\} \subseteq M_{\beta+1}$.

- Se $\beta \in \omega_{1} \backslash\{0\}$ é um ordinal limite tal que $M_{\gamma}$ foi construído para todo $\gamma \in \beta$, defina $M_{\beta}=\bigcup\left\{M_{\gamma} \mid \gamma \in \beta\right\}$.

Suponha, por absurdo, que $(X, \tau)$ não admita uma base pontualmente enumerável. Pelo lema 3.1.1(ii), $t(X) \leq \chi(X)=\omega$; assim, do lema 3.3.1, obtemos $S \subseteq \omega_{1}$ estacionário em 
$\omega_{1}$ tal que, para todo $\eta \in S$, existe $x_{\eta} \in \overline{X \cap M_{\eta}}$ tal que $\tau \cap M_{\eta}$ não contém um sistema fundamental de vizinhanças para $x_{\eta}$ em $(X, \tau)$. Como $\left\{\overline{X \cap M_{\alpha}}, \tau \cap M_{\alpha}\right\} \subseteq M_{\alpha+1}$ para todo $\alpha \in \omega_{1}$, podemos supor, pelo critério de Tarski, que $x_{\eta} \in M_{\eta+1}$ para todo $\eta \in S$.

Seja agora $M=\bigcup\left\{M_{\alpha} \mid \alpha \in \omega_{1}\right\} \prec H_{\theta}$, e tome uma outra $\epsilon$-cadeia elementar contínua $\left\{N_{\gamma} \mid \gamma \in \omega_{1}\right\}$ de $H_{\theta}$ tal que $\left\{D,\left(x_{\eta}\right)_{\eta \in S},\left(M_{\alpha}\right)_{\alpha \in \omega_{1}}\right\} \subseteq N_{0}$ e $\left|N_{\gamma}\right| \leq \omega$ para todo $\gamma \in \omega_{1}$. Considere $Z=D \cup\left\{x_{\eta} \mid \eta \in S\right\} \subseteq X \cap M$ - que é também um subespaço denso de $X$.

Afirmação: Para todo $\gamma \in \omega_{1}$, tem-se que $\tau \cap M \cap N_{\gamma}$ contém um sistema fundamental de vizinhanças para cada ponto de $\overline{X \cap M \cap N_{\gamma}} \cap Z$.

Fixe $\gamma \in \omega_{1}$ arbitrário. Pela hipótese do teorema, $Z$ admite uma base pontualmente enumerável - digamos, $\mathcal{B}$. Para cada $B \in \mathcal{B}$, fixe $V_{B} \in \tau$ tal que $B=V_{B} \cap Z$. Afirmamos que, para todo $y \in Z,\left\{V_{B} \mid B \in \mathcal{B}\right.$ e $\left.y \in V_{B}\right\}$ é um sistema fundamental de vizinhanças enumerável para $y \mathrm{em} X$. De fato, fixe $y \in Z$ e tome $\Omega \in \tau$ arbitrário com $y \in \Omega$; como $X$ verifica $T_{3}$, existe $\Omega^{\prime} \in \tau$ tal que $y \in \Omega^{\prime} \subseteq \overline{\Omega^{\prime}} \subseteq \Omega$. Tome então $B \in \mathcal{B}$ com $y \in B \subseteq \Omega^{\prime} \cap Z$. Se, por absurdo, não ocorresse $V_{B} \subseteq \Omega$, então $V_{B} \backslash \overline{\Omega^{\prime}}$ seria um aberto não-vazio de $X$; logo, como $Z$ é denso em $X$, haveria $x \in\left(V_{B} \backslash \overline{\Omega^{\prime}}\right) \cap Z=B \backslash \overline{\Omega^{\prime}} \subseteq$ $B \backslash \Omega^{\prime}=\emptyset$. Assim, $\left\{V_{B} \mid B \in \mathcal{B}\right\}$ contém um sistema fundamental de vizinhanças para $y$ em $X$, e $\left\{V_{B} \mid B \in \mathcal{B}\right.$ e $\left.y \in V_{B}\right\}$ é enumerável pois $\mathcal{B}$ é pontualmente enumerável e $y \in V_{B} \Leftrightarrow y \in B$.

Portanto, existe $\mathcal{U} \subseteq \tau$ tal que, para todo $y \in Z, \mathcal{U}_{y}=\{U \in \mathcal{U} \mid y \in U\}$ é um sistema fundamental de vizinhanças enumerável para $y$ em $(X, \tau)$. Como $\{X, \tau, Z\} \subseteq N_{\gamma}$, o critério de Tarski implica que existe um tal $\mathcal{U} \in N_{\gamma}$.

Fixe agora $z \in \overline{X \cap M \cap N_{\gamma}} \cap Z$ arbitrário e tome $W \in \tau$ com $z \in W$. Pela escolha de $\mathcal{U}$ e pelo lema 3.1.7 - dado que $z \in Z \subseteq X \cap M$-, existem $U_{1}, U_{2} \in \mathcal{U}$ e $V \in \tau \cap M$ tais que $z \in U_{1} \subseteq V \subseteq U_{2} \subseteq W$.

Note agora que $Z \cap N_{\gamma}$ é denso em $X \cap M \cap N_{\gamma}$, pois, como $Z \subseteq X \cap M$ é denso em $X$ 
— e, em particular, em $X \cap M-$, tem-se que

$$
H_{\theta} \models \forall a \in X \cap M \forall A \in \tau(a \in A \Rightarrow \exists b \in A \cap Z) ;
$$

$\operatorname{logo}$, como $N_{\gamma} \prec H_{\theta}$ e $\{X, M, \tau, Z\} \subseteq N_{\gamma}$, segue que

$$
N_{\gamma} \models \forall a \in X \cap M \forall A \in \tau(a \in A \Rightarrow \exists b \in A \cap Z),
$$

i.e., $\forall a \in X \cap M \cap N_{\gamma} \forall A \in \tau \cap N_{\gamma}\left(a \in A \Rightarrow \exists b \in A \cap Z \cap N_{\gamma}\right.$ ), o que implica o afirmado em virtude do lema 3.1.7.

Portanto, $z \in \overline{Z \cap N_{\gamma}}$, logo existe $y \in U_{1} \cap Z \cap N_{\gamma}$. Como $\{\mathcal{U}, y\} \subseteq N_{\gamma}$, então $\mathcal{U}_{y}=\{U \in$ $\mathcal{U} \mid y \in U\} \in N_{\gamma}$ e, por ser $\mathcal{U}_{y}$ um conjunto enumerável, segue do corolário 1.1.5 que $\mathcal{U}_{y} \subseteq N_{\gamma}$, de modo que $U_{1}, U_{2} \in N_{\gamma}$. Assim, como existe $V \in \tau \cap M$ tal que $U_{1} \subseteq V \subseteq U_{2}$, segue do critério de Tarski que existe $V^{\prime} \in \tau \cap M \cap N_{\gamma}$ nas mesmas condições — pois $\left\{\tau, M, U_{1}, U_{2}\right\} \subseteq N_{\gamma}-$, de modo que $z \in V^{\prime} \subseteq W$, como desejado.

Temos agora, pela primeira parte do lema 1.1.14, que $\left\{\omega_{1} \cap N_{\gamma} \mid \gamma \in \omega_{1}\right\}$ é um subconjunto c.u.b. de $\omega_{1}$. Assim, como $S$ é estacionário, existe $\gamma^{\prime} \in \omega_{1}$ tal que $\delta=\omega_{1} \cap N_{\gamma^{\prime}} \in S$. Finalmente, pela segunda parte do lema 1.1.14, tem-se que $M \cap N_{\gamma^{\prime}}=M_{\delta}, \operatorname{logo} x_{\delta} \in \overline{X \cap M_{\delta}}=$ $\overline{X \cap M \cap N_{\gamma^{\prime}}}$ é tal que $\tau \cap M_{\delta}=\tau \cap M \cap N_{\gamma^{\prime}}$ não contém um sistema fundamental de vizinhanças para $x_{\delta}$; isto contradiz a afirmação demonstrada anteriormente, pois $x_{\delta} \in \overline{X \cap M \cap N_{\gamma^{\prime}}} \cap$ $Z$.

q.e.d.

Vejamos agora que conseqüências podem ser obtidas deste teorema.

A primeira é o resultado que foi comentado no primeiro parágrafo desta seção:

Corolário 3.3.3. Seja $X \in \mathcal{T}_{1}$ enumeravelmente compacto tal que todo $Y \in[X] \leq \omega_{1}$ é regular e admite uma base pontualmente enumerável. Então X é metrizável.

Demonstração. Suponha, por absurdo, que $d(X) \geq \omega_{1}$. Pela proposição 2.2.3, existe $E=$ $\left\{x_{\alpha} \mid \alpha \in d(X)\right\} \subseteq X$ denso em $X$ e separado à esquerda. Seja $Z=\overline{\left\{x_{\alpha} \mid \alpha \in \omega_{1}\right\}} \subseteq X$. 
Temos que $Z$ encontra-se nas hipóteses do teorema 3.3.2, pois $d(Z) \leq \omega_{1}$ e $[Z]^{\leq \omega_{1}} \subseteq[X]^{\leq \omega_{1}}$; portanto, $Z$ admite uma base pontualmente enumerável, e segue então do teorema 1.2.10 que $w(Z)=\omega-$ uma vez que $Z$, por ser fechado em $X$, é enumeravelmente compacto.

Seja então $\mathcal{B}$ uma base de abertos enumerável para $Z$ e, para cada $\alpha \in \omega_{1}$, fixe $U_{\alpha} \in \mathcal{B}$ tal que $x_{\alpha} \in U_{\alpha} \subseteq Z \backslash \overline{\left\{x_{\beta} \mid \beta \in \alpha\right\}}$ - um tal $U_{\alpha}$ sempre existe, pois $E$ é separado à esquerda. Obtemos assim um absurdo, pois a função $\varphi: \omega_{1} \rightarrow \mathcal{B}$ dada por $\varphi(\alpha)=U_{\alpha}$ para todo $\alpha \in \omega_{1}$ é injetora.

Portanto, $d(X)=\omega$, e segue então do corolário 3.1 .3 que $X$ é metrizável. $\quad$ q.e.d.

A segunda conseqüência é um resultado de [32]:

Corolário 3.3.4. Seja $X$ um espaço topológico tal que $d(X) \leq \omega_{1}$ e todo $Y \in[X]^{\leq \omega_{1}}$ é regular e admite uma base pontualmente enumerável ${ }^{15}$. Então $w(X)=d(X)$.

Demonstração. Pelo teorema 3.3.2, $X$ admite uma base pontualmente enumerável, e o resultado decorre então do lema 3.1.2.

q.e.d.

A terceira - e última - conseqüência depende também do seguinte teorema, retirado de [3]:

Teorema 3.3.5. Seja X um espaço de Hausdorff localmente compacto que admite uma base pontualmente enumerável. Então $X$ é metrizável.

Demonstração. Seja $\mathcal{B}$ uma base pontualmente enumerável para $X$. Para cada $x \in X$, existem uma vizinhança compacta $V_{x}$ de $x$ e $U_{x} \in \mathcal{B}$ tais que $x \in U_{x} \subseteq V_{x}$. Pelo corolário 1.2.11, cada $V_{x}$ é metrizável e tem peso enumerável; portanto, $U_{x}$ é metrizável e separável para todo $x \in X$. Seja agora $\mathcal{B}^{\prime}=\left\{U_{x} \mid x \in X\right\}$ e defina, para $x, y \in X$ quaisquer, $x \sim y$ se, e somente se, existem $n \in \omega$ e $\left\{W_{0}, \ldots, W_{n}\right\} \subseteq \mathcal{B}^{\prime}$ tais que $x \in W_{0}, y \in W_{n}$ e, para todo $i \in n, W_{i} \cap W_{i+1} \neq \emptyset$. Nestas condições, temos que $\sim$ é uma relação de equivalência sobre $X$.

\footnotetext{
${ }^{15}$ Mais uma vez, enfraquecemos a hipótese original "é metrizável" para "é regular e admite uma base pontualmente enumerável", sem que se fizesse necessário alterar a demonstração.
} 
Fixe uma $\sim$-classe de equivalência $A \subseteq X$ arbitrária. Dado $a \in A$, tem-se pela definição de $\sim$ que $U_{a} \subseteq A, \operatorname{logo} A$ é um aberto de $X$. Fixe agora $a_{0} \in A$ e defina

$$
A_{n}=\left\{x \in X \mid \exists\left\{W_{0}, \ldots, W_{n}\right\} \subseteq \mathcal{B}^{\prime}\left(a_{0} \in W_{0} \text { e } x \in W_{n} \text { e } \forall i \in n\left(W_{i} \cap W_{i+1} \neq \emptyset\right)\right)\right\}
$$

para todo $n \in \omega$. Note que $A=\bigcup\left\{A_{n} \mid n \in \omega\right\}$.

Agora, para cada $U \in \mathcal{B}^{\prime}$, como $U$ é separável e $\mathcal{B}^{\prime} \subseteq \mathcal{B}$ é pontualmente enumerável, segue que $\left\{W \in \mathcal{B}^{\prime} \mid W \cap U \neq \emptyset\right\}$ é enumerável. Temos então, por indução em $n \in \omega$, que todo $A_{n}$ é uma união enumerável de elementos de $\mathcal{B}^{\prime}$ - e, portanto, o mesmo vale para $A-$; de fato,

- $A_{0}=\bigcup\left\{W \in \mathcal{B}^{\prime} \mid a_{0} \in W\right\}$, donde decorre o afirmado por $\mathcal{B}^{\prime}$ ser pontualmente enumerável;

- Suponha que, para um certo $n \in \omega$, a tese seja válida, de modo que $A_{n}=\bigcup \mathcal{U}_{n}$ para um certo $\mathcal{U}_{n} \subseteq \mathcal{B}^{\prime}$ enumerável. Da construção de $A_{n+1}$, temos que

$$
\begin{aligned}
A_{n+1}=\bigcup\left\{W \in \mathcal{B}^{\prime} \mid W \cap A_{n} \neq \emptyset\right\}=\bigcup\left\{W \in \mathcal{B}^{\prime} \mid W \cap \bigcup \mathcal{U}_{n} \neq \emptyset\right\}= & \\
& =\bigcup\left\{W \in \mathcal{B}^{\prime} \mid \exists U \in \mathcal{U}_{n}(W \cap U \neq \emptyset)\right\},
\end{aligned}
$$

$\log \mathrm{O}$

$$
A_{n+1}=\bigcup\left(\bigcup_{U \in \mathcal{U}_{n}}\left\{W \in \mathcal{B}^{\prime} \mid W \cap U \neq \emptyset\right\}\right)
$$

e, como $\mathcal{U}_{n}$ é enumerável e $\left\{W \in \mathcal{B}^{\prime} \mid W \cap U \neq \emptyset\right\}$ é enumerável para todo $U \in \mathcal{B}^{\prime}$, obtemos o desejado.

Assim, $A$ é uma união enumerável de conjuntos separáveis, logo é também separável e, por admitir uma base pontualmente enumerável, tem peso enumerável — pelo lema 3.1.2. Portanto, $X$ é uma união disjunta de subconjuntos abertos que admitem base enumerável; segue então do corolário 1.2.7 que $X$ é metrizável, pois, por ser $T_{2}$ e localmente compacto, é um espaço regular.

q.e.d.

Disto decorrem naturalmente os últimos resultados desta seção: 
Corolário 3.3.6. Seja $X$ um espaço de Hausdorff localmente compacto tal que $d(X) \leq \omega_{1} e$ todo $Y \in[X]^{\leq \omega_{1}}$ admite uma base pontualmente enumerável. Então $X$ é metrizável.

Demonstração. Pelo teorema 3.3.2, $\mathrm{X}$ admite uma base pontualmente enumerável ${ }^{16}$, e o resultado segue então do teorema 3.3.5.

q.e.d.

Corolário 3.3.7. Seja $X$ um espaço de Hausdorff localmente compacto tal que $d(X) \leq \omega_{1}$ e todo $Y \in[X]^{\leq \omega_{1}}$ é metrizável. Então $X$ é metrizável.

Concluiremos esta seção com uma observação presente em [2] sobre a necessidade da hipótese $d(X) \leq \omega_{1}$ no corolário 3.3.6. Foi provada em [1] a consistência da afirmação

Se $X$ é um espaço de Hausdorff localmente compacto tal que todo $Y \in[X]^{\leq \omega_{1}}$ admite uma base pontualmente enumerável, então X é metrizável.

Por outro lado, o espaço topológico $S$ construído na seção 3.2 é localmente compacto ${ }^{17}$ e não-metrizável — uma vez que não é coletivamente de Hausdorff —; além disso, por um argumento similar ao utilizado na demonstração do teorema 3.1.11, tem-se que todo $Y \in[S]^{\leq \omega_{1}}$ é metrizável — logo, em particular, admite uma base pontualmente enumerável.

Assim sendo, tem-se que a negação da afirmação em destaque acima também é consistente - e, portanto, pelo parágrafo anterior, a afirmação é independente de ZFC.

\footnotetext{
${ }^{16}$ Note que $X$ é regular, pois $X \in \mathcal{T}_{2}$ e é localmente compacto.

${ }^{17}$ Este fato segue diretamente da definição de sua topologia.
} 


\section{Apêndice A}

\section{O lema da canonização}

O objetivo deste apêndice é demonstrar o lema da canonização - devido a P. Erdôs, A. Hajnal e R. Rado -, o qual foi utilizado na demonstração do teorema 2.2.16. O enunciado do mesmo segue-se à seguinte definição:

Definição A.1. Um conjunto $\mathscr{A}$ é dito uma partição se, e somente se, $\emptyset \notin \mathscr{A} e \forall \mathcal{A}, \mathcal{B} \in$ $\mathscr{A}(\mathcal{A} \neq \mathcal{B} \Rightarrow \mathcal{A} \cap \mathcal{B}=\emptyset)$. Se $\mathcal{Z}=\bigcup \mathscr{A}$, dizemos ainda que $\mathscr{A}$ é uma partição de $\mathcal{Z}$.

Lema da canonização. Sejam $\lambda$ um cardinal limite forte e singular, $T$ um conjunto com $|T|=\lambda e \triangleleft$ uma boa-ordem sobre T. Sejam ainda $\kappa \in \lambda$ um cardinal, $n \in \omega \backslash\{0\}$ e $\mathscr{P}$ uma partição de $[T]^{n}$ com $|\mathscr{P}|=\kappa$. Finalmente, seja $p:[T]^{n} \rightarrow \mathscr{P}$ definida por $\forall A \in[T]^{n}(A \in$ $p(A))$.

Então existem $S \subseteq T$ e uma partição $\left\{S_{\xi} \mid \xi \in \sigma\right\}$ de $S$, sendo $\sigma=c f(\lambda)$, verificando as seguintes condições:

(i) $\left(\left|S_{\xi}\right|\right)_{\xi \in \sigma}$ é uma seqüência estritamente crescente de cardinais infinitos e tem limite $\lambda$;

(ii) se $\xi_{1}, \xi_{2} \in \sigma$ são tais que $\xi_{1}<\xi_{2}$, tem-se que $x_{1} \triangleleft x_{2}$ para quaisquer $x_{1} \in S_{\xi_{1}}$ e $x_{2} \in S_{\xi_{2}}$;

(iii) se $A, B \in[S]^{n}$ são tais que $\left|A \cap S_{\xi}\right|=\left|B \cap S_{\xi}\right|$ para todo $\xi \in \sigma$, então $p(A)=p(B)$. 
O principal resultado utilizado na demonstração do lema da canonização que apresentaremos - retirada de [22] — é o teorema de Erdôs-Rado, demonstrado a seguir. Antes, uma definição necessária para enunciá-lo:

Definição A.2. Dado um cardinal $\kappa$, define-se o cardinal $\exp ^{(n)} \kappa$ para $n \in \omega$ recursivamente por $\exp ^{(0)} \kappa=\kappa e, \forall n \in \omega, \exp ^{(n+1)} \kappa=2^{\left(\exp ^{(n)} \kappa\right)}$.

Teorema (Erdôs-Rado). Sejam $\kappa$ um cardinal infinito, $n \in \omega, E$ um conjunto com $|E|>$ $\exp ^{(n)} \kappa e\left\{\mathcal{P}_{\alpha} \mid \alpha \in \kappa\right\}$ uma partição de $[E]^{n+1}$. Então existem $\alpha \in \kappa$ e $D \subseteq E$ com $|D|>\kappa$ tais que $[D]^{n+1} \subseteq \mathcal{P}_{\alpha}$.

Demonstração. Procederemos por indução em $n \in \omega$. Para o caso $n=0$, basta notar que, como $\left|[E]^{1}\right|=|E|>\kappa$, deve então existir $\alpha \in \kappa$ tal que $\left|\mathcal{P}_{\alpha}\right|>\kappa$; assim, o resultado segue tomando-se $D=\bigcup \mathcal{P}_{\alpha} \subseteq E$.

Assuma agora que a afirmação seja verdadeira para um certo $n \in \omega$, e tome $E$ com $|E|>\exp ^{(n+1)} \kappa$ e uma partição $\left\{\mathcal{P}_{\alpha} \mid \alpha \in \kappa\right\}$ de $[E]^{n+2}$.

Considere, para cada ordinal $\alpha \leq\left(\exp ^{(n)} \kappa\right)^{+}$,

$$
G_{\alpha}=\prod_{\beta \in \alpha}{ }^{[\beta]^{n}} \kappa
$$

e $\left(s_{\beta}\right)_{\beta \in \gamma}=s\left\lceil\gamma \in G_{\gamma}\right.$ para $s=\left(s_{\beta}\right)_{\beta \in \alpha} \in G_{\alpha}$ e $\gamma \in \alpha$ quaisquer.

Defina $R_{\emptyset}=E$ e tome $x_{\emptyset} \in E$ arbitrário. Construiremos, indutivamente, $\left\{R_{s} \mid s \in G_{\alpha}\right\} \subseteq$ $\wp(E)$ e $\left\{x_{s} \mid s \in G_{\alpha}\right\} \subseteq E$ para cada $\alpha \leq\left(\exp ^{(n)} \kappa\right)^{+}$, procedendo da seguinte maneira:

- Para $\alpha$ sucessor - digamos, $\alpha=\gamma+1$-, defina, para cada $s=\left(s_{\beta}\right)_{\beta \in \alpha} \in G_{\alpha}$,

$$
R_{s}=\left\{y \in R_{s \mid \gamma} \backslash\left\{x_{s \mid \gamma}\right\} \mid \forall\left\{\beta_{1}, \ldots, \beta_{n}\right\} \in[\gamma]^{n}\left(\left\{x_{s \mid \beta_{1}}, \ldots, x_{s\left\lceil\beta_{n}\right.}, x_{s \mid \gamma}, y\right\} \in \mathcal{P}_{s_{\gamma}\left(\left\{\beta_{1}, \ldots, \beta_{n}\right\}\right)}\right)\right\} .
$$

- Se $\alpha \neq 0$ é um ordinal limite, defina $R_{s}=\bigcap\left\{R_{s \mid \beta} \mid \beta \in \alpha\right\}$ para cada $s \in G_{\alpha}$.

- Em ambos os casos, para cada $s \in G_{\alpha}$, tome $x_{s}=x_{\emptyset}$ se $R_{s}=\emptyset$ e $x_{s} \in R_{s}$ arbitrário caso contrário. 
Afirmação 1: Dados $\alpha, \beta \leq\left(\exp ^{(n)} \kappa\right)^{+} \operatorname{com} \beta<\alpha$ e $s \in G_{\alpha}$ arbitrários, tem-se que $R_{s \uparrow \beta} \supseteq R_{s}$ e $x_{s \uparrow \beta} \notin R_{s}$.

Provaremos a primeira parte por indução transfinita em $\alpha$.

- Para $\alpha=0$, nada há a se fazer.

- Suponha que a afirmação seja verdadeira para um certo $\alpha \leq\left(\exp ^{(n)} \kappa\right)^{+}$e tome $\beta \in(\alpha+1)$ e $s \in G_{\alpha+1}$ arbitrários. Por construção, tem-se que $R_{s\lceil\alpha} \supseteq R_{s}$; assim, como $\beta \leq \alpha$, segue da hipótese de indução que $R_{s \uparrow \beta} \supseteq R_{s \uparrow \alpha} \supseteq R_{s}$.

- Se $\alpha \neq 0$ é um ordinal limite e $\beta \in \alpha$, é imediato pela construção que $R_{s \uparrow \beta} \supseteq$ $\bigcap\left\{R_{s \uparrow \beta} \mid \beta \in \alpha\right\}=R_{s}$.

A segunda parte segue então do fato de que, novamente por construção, $x_{s \uparrow \beta} \notin R_{s \uparrow(\beta+1)} \supseteq$ $R_{s}$.

Afirmação 2: Fixe $\alpha \leq\left(\exp ^{(n)} \kappa\right)^{+}$arbitrário. Para quaisquer $s, t \in G_{\alpha}$ distintos, tem-se que $R_{s} \cap R_{t}=\emptyset$.

Considere $s=\left(s_{\beta}\right)_{\beta \in \alpha}$ e $t=\left(t_{\beta}\right)_{\beta \in \alpha}$ elementos distintos de $G_{\alpha}$ e seja $\gamma=\min \{\beta \in$ $\left.\alpha \mid s_{\beta} \neq t_{\beta}\right\}$. Então existe $\left\{\beta_{1}, \ldots, \beta_{n}\right\} \in[\gamma]^{n}$ tal que $s_{\gamma}\left(\left\{\beta_{1}, \ldots, \beta_{n}\right\}\right) \neq t_{\gamma}\left(\left\{\beta_{1}, \ldots, \beta_{n}\right\}\right)$, $\log 0$

$$
R_{s} \subseteq R_{s \uparrow(\gamma+1)} \subseteq\left\{y \in R_{s\lceil\gamma} \mid\left\{x_{s\left\lceil\beta_{1}\right.}, \ldots, x_{s \uparrow \beta_{n}}, x_{s\lceil\gamma}, y\right\} \in \mathcal{P}_{s_{\gamma}\left(\left\{\beta_{1}, \ldots, \beta_{n}\right\}\right)}\right\}=: E_{s}
$$

e

$$
R_{t} \subseteq R_{t \uparrow(\gamma+1)} \subseteq\left\{y \in R_{t \uparrow \gamma} \mid\left\{x_{t \uparrow \beta_{1}}, \ldots, x_{t \uparrow \beta_{n}}, x_{t \uparrow \gamma}, y\right\} \in \mathcal{P}_{t_{\gamma}\left(\left\{\beta_{1}, \ldots, \beta_{n}\right\}\right)}\right\}=: E_{t}
$$

Pela minimalidade de $\gamma$, temos que $s \uparrow \gamma=t \uparrow \gamma$ e $s\left\lceil\beta_{i}=t \uparrow \beta_{i}\right.$ para todo $i \in\{1, \ldots, n\}$; assim,

$$
E_{t}=\left\{y \in R_{s\lceil\gamma} \mid\left\{x_{s \uparrow \beta_{1}}, \ldots, x_{s \uparrow \beta_{n}}, x_{s\lceil\gamma}, y\right\} \in \mathcal{P}_{t_{\gamma}\left(\left\{\beta_{1}, \ldots, \beta_{n}\right\}\right)}\right\}
$$

e segue então da escolha de $\left\{\beta_{1}, \ldots, \beta_{n}\right\}$ que $E_{s} \cap E_{t}=\emptyset$. Portanto, $R_{s} \cap R_{t}=\emptyset$. 
Afirmação 3: Para todo $\alpha \leq\left(\exp ^{(n)} \kappa\right)^{+}$, tem-se

$$
\bigcup_{s \in G_{\alpha}} R_{s}=E \backslash \bigcup_{\beta \in \alpha}\left\{x_{t} \mid t \in G_{\beta}\right\} .
$$

Demonstraremos esta afirmação por indução transfinita em $\alpha$.

- Se $\alpha=0$, a afirmação é trivialmente verdadeira.

- Suponha que a afirmação seja verdadeira para um certo $\alpha \in\left(\exp ^{(n)} \kappa\right)^{+}$. Para uma inclusão, suponha, por absurdo, que exista

$$
y \in\left(\bigcup_{s \in G_{\alpha+1}} R_{s}\right) \cap\left(\bigcup_{\beta \in \alpha+1}\left\{x_{t} \mid t \in G_{\beta}\right\}\right),
$$

e sejam $\beta \in \alpha+1$ e $t \in G_{\beta}$ tais que $y=x_{t}$. Se ocorresse $\beta<\alpha$, seguiria da hipótese de indução que

$$
y \notin \bigcup_{s^{\prime} \in G_{\alpha}} R_{s^{\prime}} \supseteq \bigcup_{s \in G_{\alpha+1}} R_{s}
$$

- pois, por construção, tem-se que

$$
R_{s} \subseteq R_{s\lceil\alpha} \subseteq \bigcup_{s^{\prime} \in G_{\alpha}} R_{s^{\prime}}
$$

para todo $s \in G_{\alpha+1}-$, o que é falso. Assim, $\beta=\alpha$ e, portanto, de

$$
y=x_{t} \in \bigcup_{s \in G_{\alpha+1}} R_{s} \subseteq \bigcup_{s \in G_{\alpha+1}} R_{s\lceil\alpha} \backslash\left\{x_{s\lceil\alpha}\right\}
$$

segue que

$$
y \in \bigcup_{\substack{s \in G_{\alpha+1} \\ s\lceil\alpha \neq t}} R_{s\lceil\alpha} \backslash\left\{x_{s \uparrow \alpha}\right\}
$$

o que é um absurdo, pois $y=x_{t} \in R_{t}$ e, pela afirmação $2, R_{t} \cap R_{s^{\prime}}=\emptyset$ para todo $s^{\prime} \in G_{\alpha} \backslash\{t\}$. Logo,

$$
\bigcup_{s \in G_{\alpha+1}} R_{s} \subseteq E \backslash \bigcup_{\beta \in \alpha+1}\left\{x_{t} \mid t \in G_{\beta}\right\}
$$


Para a outra inclusão, note que

$$
E \backslash \bigcup_{\beta^{\prime} \in \alpha}\left\{x_{t} \mid t \in G_{\beta^{\prime}}\right\} \supseteq E \backslash \bigcup_{\beta \in \alpha+1}\left\{x_{t} \mid t \in G_{\beta}\right\}
$$

e, como já foi observado,

$$
\bigcup_{s^{\prime} \in G_{\alpha}} R_{s^{\prime}} \supseteq \bigcup_{s \in G_{\alpha+1}} R_{s}
$$

Pela hipótese de indução,

$$
\bigcup_{s^{\prime} \in G_{\alpha}} R_{s^{\prime}}=E \backslash \bigcup_{\beta^{\prime} \in \alpha}\left\{x_{t} \mid t \in G_{\beta^{\prime}}\right\} ;
$$

portanto, a inclusão desejada é equivalente a

$$
\left(\bigcup_{s^{\prime} \in G_{\alpha}} R_{s^{\prime}}\right) \backslash\left(\bigcup_{s \in G_{\alpha+1}} R_{s}\right) \subseteq\left\{x_{t} \mid t \in G_{\alpha}\right\} .
$$

Tome então

$$
y \in\left(\bigcup_{s^{\prime} \in G_{\alpha}} R_{s^{\prime}}\right) \backslash\left(\bigcup_{s \in G_{\alpha+1}} R_{s}\right),
$$

e seja $s_{0}^{\prime} \in G_{\alpha}$ tal que $y \in R_{s_{0}^{\prime}}$. Suponha, por absurdo, que $y \neq x_{s_{0}^{\prime}}$, e tome $\tilde{s} \in G_{\alpha+1}$ definida por $\tilde{s}\left\lceil\alpha=s_{0}^{\prime}\right.$ e, para todo $\left\{\beta_{1}, \ldots, \beta_{n}\right\} \in[\alpha]^{n}$,

$$
\left\{x_{s_{0}^{\prime} \mid \beta_{1}}, \ldots, x_{s_{0}^{\prime} \mid \beta_{n}}, x_{s_{0}^{\prime}}, y\right\} \in \mathcal{P}_{\tilde{s}_{\alpha}\left(\left\{\beta_{1}, \ldots, \beta_{n}\right\}\right)}
$$

- note que, em virtude da segunda parte da afirmação 1 , o conjunto $\left\{x_{s_{0}^{\prime} \mid \beta_{1}}, \ldots\right.$, $\left.x_{s_{0}^{\prime} \mid \beta_{n}}, x_{s_{0}^{\prime}}, y\right\}$ tem, de fato, $n+2$ elementos. Por hipótese, $y \notin R_{\tilde{s}}$; logo, como supusemos que $y \neq x_{s_{0}^{\prime}}$, existe $\left\{\tilde{\beta}_{1}, \ldots, \tilde{\beta}_{n}\right\} \in[\alpha]^{n}$ tal que

$$
\left\{x_{\tilde{s} \mid \tilde{\beta}_{1}}, \ldots, x_{\tilde{s} \mid \tilde{\beta}_{n}}, x_{\tilde{s} \mid \alpha}, y\right\} \notin \mathcal{P}_{\tilde{s}_{\alpha}\left(\left\{\tilde{\beta}_{1}, \ldots, \tilde{\beta}_{n}\right\}\right)},
$$

contradizendo a definição de $\tilde{s}$. Portanto, $y=x_{s_{0}^{\prime}} \in\left\{x_{t} \mid t \in G_{\alpha}\right\}$. 
- Finalmente, suponha que $\alpha \neq 0$ seja um ordinal limite tal que a afirmação é verdadeira para todo $\gamma \in \alpha$. Como

$$
E \backslash \bigcup_{\beta \in \alpha}\left\{x_{t} \mid t \in G_{\beta}\right\}=\bigcap_{\gamma \in \alpha}\left(E \backslash \bigcup_{\beta \in \gamma}\left\{x_{t^{\prime}} \mid t^{\prime} \in G_{\beta}\right\}\right)
$$

e, para todo $\gamma \in \alpha$,

$$
\bigcup_{s^{\prime} \in G_{\gamma}} R_{s^{\prime}}=E \backslash \bigcup_{\beta \in \gamma}\left\{x_{t^{\prime}} \mid t^{\prime} \in G_{\beta}\right\}
$$

a igualdade requerida é então equivalente a

$$
\bigcup_{s \in G_{\alpha}} R_{s}=\bigcap_{\gamma \in \alpha}\left(\bigcup_{s^{\prime} \in G_{\gamma}} R_{s^{\prime}}\right) \text {. }
$$

Para a primeira inclusão, tome $y \in \bigcup_{s \in G_{\alpha}} R_{s}$ arbitrário, e seja $s \in G_{\alpha}$ tal que $y \in R_{s}$. Pela primeira parte da afirmação 1 , temos que $y \in R_{s\lceil\gamma} \subseteq \bigcup_{s^{\prime} \in G_{\gamma}} R_{s^{\prime}}$ para todo $\gamma \in \alpha$, donde segue o desejado.

Para a outra inclusão, tome

$$
y \in \bigcap_{\gamma \in \alpha}\left(\bigcup_{s^{\prime} \in G_{\gamma}} R_{s^{\prime}}\right)
$$

arbitrário e, para cada $\gamma \in \alpha$, fixe $s_{\gamma}^{\prime} \in G_{\gamma}$ tal que $y \in R_{s_{\gamma}^{\prime}}$. Se, por absurdo, houvesse $\gamma_{1}, \gamma_{2} \in \alpha$ tais que $\gamma_{1}<\gamma_{2}$ e $s_{\gamma_{2}}^{\prime} \uparrow \gamma_{1} \neq s_{\gamma_{1}}^{\prime}$, teríamos, pela primeira parte da afirmação 1 , que $y \in R_{s_{\gamma_{1}}^{\prime}} \cap R_{s_{\gamma_{2}}^{\prime}} \subseteq R_{s_{\gamma_{1}}^{\prime}} \cap R_{s_{\gamma_{2}}^{\prime} \mid \gamma_{1}}$, contradizendo a afirmação 2 . Portanto, podemos definir $\tilde{s} \in G_{\alpha}$ por $\forall \gamma \in \alpha\left(\tilde{s} \uparrow \gamma=s_{\gamma}^{\prime}\right)$ e, assim, como $y \in R_{\tilde{s}\lceil\gamma}$ para todo $\gamma \in \alpha$, segue que

$$
y \in \bigcap_{\gamma \in \alpha} R_{\tilde{s}\lceil\gamma}=R_{\tilde{s}} \subseteq \bigcup_{s \in G_{\alpha}} R_{s} .
$$

Seja agora $\nu=\left(\exp ^{(n)} \kappa\right)^{+}$. Temos que

$$
\left|\bigcup_{\beta \in \nu}\left\{x_{t} \mid t \in G_{\beta}\right\}\right| \leq \sum_{\beta \in \nu}\left|G_{\beta}\right| \leq \sum_{\beta \in \nu}\left(\sum_{\gamma \in \beta} \kappa^{|\gamma|}\right) \leq \sum_{\beta \in \nu}|\beta| \cdot \kappa^{|\beta|} \leq \sum_{\beta \in \nu} \kappa^{|\beta|} \leq
$$




$$
\leq \nu \cdot \kappa^{\left(\exp ^{(n)} \kappa\right)} \leq\left(\exp ^{(n)} \kappa\right)^{+} \cdot\left(2^{\kappa}\right)^{\left(\exp ^{(n)} \kappa\right)} \leq 2^{\left(\exp ^{(n)} \kappa\right)}=\exp ^{(n+1)} \kappa<|E| ;
$$

logo, pela afirmação 3 ,

$$
\bigcup_{s \in G_{\nu}} R_{s}=E \backslash \bigcup_{\beta \in \nu}\left\{x_{t} \mid t \in G_{\beta}\right\} \neq \emptyset,
$$

de modo que existe $\tilde{s}=\left(\tilde{s}_{\beta}\right)_{\beta \in \nu} \in G_{\nu}$ tal que $R_{\tilde{s}} \neq \emptyset$.

Defina agora uma partição $\left\{\mathcal{B}_{\alpha} \mid \alpha \in \kappa\right\}$ de $[\nu]^{n+1}$ por

$$
\left\{\beta_{1}, \ldots, \beta_{n+1}\right\} \in \mathcal{B}_{\alpha} \Leftrightarrow \tilde{s}_{\beta_{n+1}}\left(\left\{\beta_{1}, \ldots, \beta_{n}\right\}\right)=\alpha
$$

para quaisquer $\alpha \in \kappa$ e $\beta_{1}, \ldots, \beta_{n+1} \in \nu \operatorname{com} \beta_{1}<\ldots<\beta_{n+1}$. Pela hipótese de indução, existem $\tilde{\alpha} \in \kappa$ e $H \subseteq \nu$ com $|H|>\kappa$ tais que $[H]^{n+1} \subseteq \mathcal{B}_{\tilde{\alpha}}$.

Tome então $D=\left\{x_{\tilde{s} \uparrow \beta} \mid \beta \in H\right\} \subseteq E$. Pela segunda parte da afirmação 1, temos que $x_{\tilde{s} \mid \beta_{1}} \neq x_{\tilde{s}\left\lceil\beta_{2}\right.}$ para quaisquer $\beta_{1}, \beta_{2} \in \nu$ distintos, $\operatorname{logo}|D|=|H|>\kappa$. Além disso, dado $\left\{x_{\tilde{s} \mid \beta_{1}}, \ldots, x_{\tilde{s} \mid \beta_{n+2}}\right\} \in[D]^{n+2}$ arbitrário com $\beta_{1}<\ldots<\beta_{n+2}$, segue da primeira parte da afirmação 1 que $x_{\tilde{s} \mid \beta_{n+2}} \in R_{\tilde{s} \mid \beta_{n+2}} \subseteq R_{\tilde{s} \mid\left(\left(\beta_{n+1}\right)+1\right)}$; logo, da definição de $R_{\tilde{s} \mid\left(\left(\beta_{n+1}\right)+1\right)}$, temos que

$$
\left\{x_{\tilde{s} \mid \beta_{1}}, \ldots, x_{\tilde{s}\left\lceil\beta_{n}\right.}, x_{\tilde{s}\left\lceil\beta_{n+1}\right.}, x_{\tilde{s} \mid \beta_{n+2}}\right\} \in \mathcal{P}_{\tilde{s}_{\beta_{n+1}}}\left(\left\{\beta_{1}, \ldots, \beta_{n}\right\}\right)
$$

e, como $\left\{\beta_{1}, \ldots, \beta_{n+1}\right\} \in[H]^{n+1} \subseteq \mathcal{B}_{\tilde{\alpha}}$, segue que $\tilde{s}_{\beta_{n+1}}\left(\left\{\beta_{1}, \ldots, \beta_{n}\right\}\right)=\tilde{\alpha}$, o que acarreta $\left\{x_{\tilde{s} \mid \beta_{1}}, \ldots, x_{\tilde{s} \mid \beta_{n+2}}\right\} \in \mathcal{P}_{\tilde{\alpha}}$. Portanto, $[D]^{n+2} \subseteq \mathcal{P}_{\tilde{\alpha}}$.

Precisaremos agora de mais dois lemas para proceder à demonstração do resultado principal.

Lema A.3. Sejam A um conjunto, $\theta$ e $\kappa$ cardinais e $\left\{\sim_{\alpha} \mid \alpha \in \theta\right\}$ um conjunto de relações de equivalência sobre $A$ tais que, para cada $\alpha \in \theta$, a relação $\sim_{\alpha}$ determina no máximo $\kappa$ classes de equivalência em A. Então a relação de equivalência $\sim$ definida em A por

$$
x \sim y \Leftrightarrow \forall \alpha \in \theta\left(x \sim_{\alpha} y\right)
$$

determina no máximo $\kappa^{\theta}$ classes de equivalência em $A$. 
Demonstração. Para cada $\alpha \in \theta$, seja $\left\{A_{\eta}^{\alpha} \mid \eta \in \kappa\right\}$ o conjunto das classes de equivalência determinadas por $\sim_{\alpha}$ em $A$; caso $\sim_{\alpha}$ determine $\rho<\kappa$ classes, ordene-as por $\left\{A_{\eta}^{\alpha} \mid \eta \in \rho\right\}$ e defina $A_{\eta}^{\alpha}=\emptyset$ para $\eta \in\left[\rho, \kappa\left[\right.\right.$. Seja agora $\varphi:{ }^{\theta} \kappa \rightarrow \wp(A)$ definida por

$$
\varphi(f)=\bigcap_{\alpha \in \theta} A_{f(\alpha)}^{\alpha}
$$

para toda $f \in{ }^{\theta} \kappa$.

Note que, dada $f \in{ }^{\theta} \kappa$ arbitrária, se $x, y \in \varphi(f)$ então $x \sim y$. Assim, sendo $\mathcal{E} \subseteq \wp(A)$ o conjunto das classes de equivalência que $\sim$ determina em $A$, temos que, para toda $f \in{ }^{\theta} \kappa$, existe $E_{f} \in \mathcal{E}$ tal que $\varphi(f) \subseteq E_{f}$. Tome então

$$
\begin{aligned}
\psi:{ }^{\theta} \kappa & \rightarrow \mathcal{E} \\
f & \mapsto E_{f} .
\end{aligned}
$$

Afirmamos que $\psi$ é uma função sobrejetora.

De fato, fixe $x \in A$ arbitrário. Para todo $\alpha \in \theta$, existe $\eta_{x, \alpha} \in \kappa$ tal que $x \in A_{\eta_{x, \alpha}}^{\alpha}$; defina agora $f_{x}: \theta \rightarrow \kappa$ por $f_{x}(\alpha)=\eta_{x, \alpha}$ para todo $\alpha \in \theta$, e teremos então que

$$
x \in \bigcap_{\alpha \in \theta} A_{f_{x}(\alpha)}^{\alpha}=\varphi\left(f_{x}\right) \subseteq E_{f_{x}}=\psi\left(f_{x}\right) .
$$

Assim, para todo $x \in A$, existe $f_{x} \in{ }^{\theta} \kappa$ tal que $\psi\left(f_{x}\right) \in \psi\left[{ }^{\theta} \kappa\right]$ é a $\sim$-classe de equivalência em $A$ à qual $x$ pertence. Isto implica que $\psi$ é sobrejetora, o que, por sua vez, implica que $|\mathcal{E}| \leq \kappa^{\theta}$.

q.e.d.

Antes de enunciar o segundo — e último - lema, é necessário apresentar mais duas definições:

Definição A.4. Sejam $\lambda$ um cardinal infinito, $T$ um conjunto com $|T|=\lambda$ e uma ordem linear sobre $T$. Sejam ainda $\kappa \in \lambda$ um cardinal, $n \in \omega$ e $\mathscr{P}$ uma partição de $[T]^{n}$ com $|\mathscr{P}|=\kappa$. Finalmente, seja $p:[T]^{n} \rightarrow \mathscr{P}$ definida por $\forall A \in[T]^{n}(A \in p(A))$. 
Fixe agora $r \in\{1, \ldots, n\}$ e $n_{1}, \ldots, n_{r} \in\{1, \ldots, n\}$ tais que $n_{1}+\ldots+n_{r}=n$, e sejam $\sigma=c f(\lambda), k \in\{0, \ldots, r\}$ e $\left\{S_{\xi} \mid \xi \in \sigma\right\} \subseteq \wp(T)$.

Dizemos que $\left\{S_{\xi} \mid \xi \in \sigma\right\}$ é um sistema $\left(n_{1}, \ldots, n_{r}, k\right)$-canônico com respeito a $\mathscr{P}$ se, $e$ somente se, as seguintes condições são satisfeitas:

(i) $\left(\left|S_{\xi}\right|\right)_{\xi \in \sigma}$ é uma seqüência estritamente crescente de cardinais infinitos e tem limite $\lambda$;

(ii) se $\xi_{1}, \xi_{2} \in \sigma$ são tais que $\xi_{1}<\xi_{2}$, tem-se que $x_{1} \triangleleft x_{2}$ para quaisquer $x_{1} \in S_{\xi_{1}}$ e $x_{2} \in S_{\xi_{2}}$;

(iii) se $A, B \in\left[\bigcup\left\{S_{\xi} \mid \xi \in \sigma\right\}\right]^{n}$ são tais que existem $\xi_{1}, \ldots, \xi_{r} \in \sigma$ satisfazendo

$\cdot \xi_{1}<\ldots<\xi_{r}$

- $A \cap S_{\xi_{i}}=B \cap S_{\xi_{i}}$ para todo $i \in\{1, \ldots, k\}$

$e$

$\cdot\left|A \cap S_{\xi_{i}}\right|=\left|B \cap S_{\xi_{i}}\right|=n_{i}$ para todo $i \in\{1, \ldots, r\}$,

então $p(A)=p(B)$.

Definição A.5. Sejam $\mathscr{A}$ e $\mathscr{B}$ partições. Dizemos que $\mathscr{A}$ é um refinamento de $\mathscr{B}$ se, e somente se, existe $\varphi: \mathscr{A} \rightarrow \mathscr{B}$ injetora tal que $\mathcal{A} \subseteq \varphi(\mathcal{A})$ para todo $\mathcal{A} \in \mathscr{A}$.

Lema A.6. Considere as condições da definição A.4 e inclua a hipótese de que $\lambda$ é um cardinal limite forte e singular. Se $k \neq 0$, então todo sistema $\left(n_{1}, \ldots, n_{r}, k\right)$-canônico com respeito a $\mathscr{P}$ admite um refinamento que é um sistema $\left(n_{1}, \ldots, n_{r}, k-1\right)$-canônico com respeito a $\mathscr{P}$.

Demonstração. Suponha que $k \neq 0$ e seja $\left\{S_{\xi} \mid \xi \in \sigma\right\}$ um sistema $\left(n_{1}, \ldots, n_{r}, k\right)$-canônico com respeito a $\mathscr{P}$. Para cada $\xi \in \sigma$ e cada $n^{\prime} \in n+1$, fixe um $S_{\xi}^{n^{\prime}} \in\left[S_{\xi}\right]^{n^{\prime}}$.

Vamos construir indutivamente $\left\{S_{\xi}^{\prime} \mid \xi \in \sigma\right\}$ e $f: \sigma \rightarrow \sigma$ tais que, para todo $\xi \in \sigma$, ocorram $S_{\xi}^{\prime} \subseteq S_{f(\xi)}$ e $\left|S_{\xi}^{\prime}\right|>\left|S_{\xi}\right|$.

Suponha que, para um certo $\alpha \in \sigma$, já tenhamos construído $\left\{S_{\xi}^{\prime} \mid \xi \in \alpha\right\}$ e $f\lceil\alpha: \alpha \rightarrow \sigma$. Seja

$$
\nu=\left|S_{\alpha}\right| \cdot \exp \left(\kappa \cdot \sigma \cdot \sup _{\xi \in \alpha}\left|S_{\xi}^{\prime}\right|\right)<\lambda
$$


Como $\alpha<\sigma$ e $\sigma=c f(\lambda)$ é regular, então $\sup \{f(\xi) \mid \xi \in \alpha\}<\sigma$; tome então $f(\alpha) \in \sigma$ tal que $\sup \left\{(f\lceil\alpha)(\xi) \mid \xi \in \alpha\}<f(\alpha)\right.$ e $\left|S_{f(\alpha)}\right|>\exp ^{(n)} \nu$.

Agora, para cada $F \in\left[\bigcup\left\{S_{\xi}^{\prime} \mid \xi \in \alpha\right\}\right]^{n_{1}+\ldots+n_{k-1}}$ e cada $\varphi:\{k+1, \ldots, r\} \rightarrow \sigma$ tal que $f(\alpha)<\varphi(k+1)<\ldots<\varphi(r)<\sigma$, defina em $\left[S_{f(\alpha)}\right]^{n_{k}}$ a relação de equivalência $\sim_{F, \varphi}$ por

$$
X \sim_{F, \varphi} Y \Leftrightarrow p\left(F \cup X \cup \bigcup_{i=k+1}^{r} S_{\varphi(i)}^{n_{i}}\right)=p\left(F \cup Y \cup \bigcup_{i=k+1}^{r} S_{\varphi(i)}^{n_{i}}\right)
$$

Como cada uma destas relações determina no máximo $\kappa$ classes de equivalência em $\left[S_{f(\alpha)}\right]^{n_{k}}$ e há no máximo

$$
\theta=\sigma^{s-k} \cdot\left(\sum_{\xi \in \alpha}\left|S_{\xi}^{\prime}\right|\right)^{n_{1}+\ldots+n_{k-1}} \leq \sigma \cdot|\alpha| \cdot \sup _{\xi \in \alpha}\left|S_{\xi}^{\prime}\right| \leq \sigma \cdot \sup _{\xi \in \alpha}\left|S_{\xi}^{\prime}\right|<\lambda
$$

tais relações, segue do lema A.3 que existe uma partição $\mathscr{R}$ de $\left[S_{f(\alpha)}\right]^{n_{k}} \operatorname{com}|\mathscr{R}| \leq \kappa^{\theta} \leq$ $\left(2^{\kappa}\right)^{\theta}=2^{\kappa \cdot \theta} \leq \nu<\lambda$ que refina o conjunto de todas as classes de equivalência consideradas.

Agora, pelo teorema de Erdôs-Rado, existem $\mathcal{R} \in \mathscr{R}$ e $S_{\alpha}^{\prime} \subseteq S_{f(\alpha)} \operatorname{com}\left|S_{\alpha}^{\prime}\right|>\nu \geq\left|S_{\alpha}\right|$ tais que $\left[S_{\alpha}^{\prime}\right]^{n_{k}} \subseteq \mathcal{R}$, ou seja, tais que todos os elementos de $\left[S_{\alpha}^{\prime}\right]^{n_{k}}$ são equivalentes com respeito a todas as relações $\sim_{F, \varphi}$ consideradas. Isto conclui o passo indutivo da construção.

Provemos agora que $\left\{S_{\xi}^{\prime} \mid \xi \in \sigma\right\}$ é um sistema $\left(n_{1}, \ldots, n_{r}, k-1\right)$-canônico com respeito a $\mathscr{P}$. A condição $(i)$ da definição A.4 decorre do fato de que $\left|S_{\xi}^{\prime}\right|>\left|S_{\xi}\right|$ para todo $\xi \in \sigma$, e a condição (ii) é satisfeita pois, por construção, $f$ é uma função estritamente crescente. Resta então verificar a condição $(i i i)$.

Para tanto, sejam $A, B \in\left[\bigcup\left\{S_{\xi}^{\prime} \mid \xi \in \sigma\right\}\right]^{n}$ e $\xi_{1}, \ldots, \xi_{r} \in \sigma$ tais que $\xi_{1}<\ldots<\xi_{r}$, $A \cap S_{\xi_{i}}^{\prime}=B \cap S_{\xi_{i}}^{\prime}$ para todo $i \in\{1, \ldots, k-1\}$ e $\left|A \cap S_{\xi_{i}}^{\prime}\right|=\left|B \cap S_{\xi_{i}}^{\prime}\right|=n_{i}$ para todo $i \in\{1, \ldots, r\}$. Temos

$$
\left(\bigcup_{i=1}^{k-1}\left(A \cap S_{\xi_{i}}^{\prime}\right)\right) \cup\left(A \cap S_{\xi_{k}}^{\prime}\right) \cup\left(\bigcup_{i=k+1}^{r}\left(A \cap S_{\xi_{i}}^{\prime}\right)\right)=A \in p(A)
$$

$\log \mathrm{O}$ 


$$
\left(\bigcup_{i=1}^{k-1}\left(A \cap S_{\xi_{i}}^{\prime}\right)\right) \cup\left(A \cap S_{\xi_{k}}^{\prime}\right) \cup\left(\bigcup_{i=k+1}^{r} S_{f\left(\xi_{i}\right)}^{n_{i}}\right) \in p(A),
$$

pois $f$ é estritamente crescente, $A \cap S_{\xi_{i}}^{\prime}=A \cap S_{f\left(\xi_{i}\right)}$ para todo $i \in\{1, \ldots, r\}$ e $\left\{S_{\xi} \mid \xi \in \sigma\right\}$ é um sistema $\left(n_{1}, \ldots, n_{r}, k\right)$-canônico com respeito a $\mathscr{P}$. Pela escolha de $S_{\xi_{k}}^{\prime}$, temos que $A \cap S_{\xi_{k}}^{\prime} \sim_{F, \varphi} B \cap S_{\xi_{k}}^{\prime}$ para $F=\bigcup_{i=1}^{k-1}\left(A \cap S_{\xi_{i}}^{\prime}\right)=\bigcup_{i=1}^{k-1}\left(B \cap S_{\xi_{i}}^{\prime}\right)$ e $\varphi:\{k+1, \ldots, r\} \rightarrow \sigma$ definida por $\varphi(i)=f\left(\xi_{i}\right)$ para todo $i \in\{k+1, \ldots, r\}$; portanto,

$$
\left(\bigcup_{i=1}^{k-1}\left(B \cap S_{\xi_{i}}^{\prime}\right)\right) \cup\left(B \cap S_{\xi_{k}}^{\prime}\right) \cup\left(\bigcup_{i=k+1}^{r} S_{f\left(\xi_{i}\right)}^{n_{i}}\right) \in p(A)
$$

e, novamente por ser $\left\{S_{\xi} \mid \xi \in \sigma\right\}$ um sistema $\left(n_{1}, \ldots, n_{r}, k\right)$-canônico com respeito a $\mathscr{P}$ agora observando que, para todo $i \in\{1, \ldots, r\}$, tem-se $B \cap S_{\xi_{i}}^{\prime}=B \cap S_{f\left(\xi_{i}\right)}$ 一, obtemos

$$
B=\left(\bigcup_{i=1}^{k-1}\left(B \cap S_{\xi_{i}}^{\prime}\right)\right) \cup\left(B \cap S_{\xi_{k}}^{\prime}\right) \cup\left(\bigcup_{i=k+1}^{r}\left(B \cap S_{\xi_{i}}^{\prime}\right)\right) \in p(A)
$$

Assim, $p(A)=p(B)$, satisfazendo a condição $(i i i)$.

q.e.d.

Estamos agora em condições de, finalmente, provar o lema da canonização.

Demonstração do lema da canonização. Tome uma seqüência estritamente crescente de cardinais infinitos $\left(\mu_{\xi}\right)_{\xi \in \sigma}$ tal que $\sup \left\{\mu_{\xi} \mid \xi \in \sigma\right\}=\lambda$. Construa $\left\{\widetilde{S}_{\xi} \mid \xi \in \sigma\right\}$ indutivamente tomando, para todo $\xi \in \sigma, \widetilde{S}_{\xi} \subseteq T$ como sendo o segmento inicial de $T \backslash \bigcup\left\{\widetilde{S}_{\alpha} \mid \alpha \in \xi\right\}$ que tem tipo-de-ordem $\mu_{\xi}$. Nestas condições, $\left\{\widetilde{S}_{\xi} \mid \xi \in \sigma\right\}$ é um sistema $\left(n_{1}, \ldots, n_{r}, r\right)$ canônico com respeito a $\mathscr{P}$ para quaisquer $r \in\{1, \ldots, n\}$ e $n_{1}, \ldots, n_{r} \in\{1, \ldots, n\}$ satisfazendo $n_{1}+\ldots+n_{r}=n$.

Note agora que, dados $k \in\{0, \ldots, r\}$ e $n_{1}, \ldots, n_{r} \in\{1, \ldots, n\}$ com $n_{1}+\ldots+n_{r}=n$, se $\left\{S_{\xi}^{\prime} \mid \xi \in \sigma\right\}$ é um sistema $\left(n_{1}, \ldots, n_{r}, k\right)$-canônico com respeito a $\mathscr{P}$ e $\left\{S_{\xi}^{\prime \prime} \mid \xi \in \sigma\right\}$ é um refinamento de $\left\{S_{\xi}^{\prime} \mid \xi \in \sigma\right\}$ que verifica as condições $(i)$ e $(i i)$ da definição A.4, então $\left\{S_{\xi}^{\prime \prime} \mid \xi \in \sigma\right\}$ é também um sistema $\left(n_{1}, \ldots, n_{r}, k\right)$-canônico com respeito a $\mathscr{P}$. 
Pelo lema A.6, existe um sistema $(n, 0)$-canônico com respeito a $\mathscr{P}$ - digamos, $\left\{S_{\xi}^{1} \mid \xi \in\right.$ $\sigma\}$ - que é um refinamento de $\left\{\widetilde{S}_{\xi} \mid \xi \in \sigma\right\}$. Agora, para todo $r \in\{1, \ldots, n\}$, defina $\mathcal{N}_{r}=$ $\left\{\left(n_{1}, \ldots, n_{r}\right) \mid n_{1}, \ldots, n_{r} \in\{1, \ldots, n\}\right.$ e $\left.n_{1}+\ldots+n_{r}=n\right\}$ e $m_{r}=\left|\mathcal{N}_{r}\right|<\omega$. Recursivamente, para todo $r \in\{2, \ldots, n\}$, temos que, aplicando $r \cdot m_{r}$ vezes o lema A.6 - sendo $r$ vezes para cada $\left(n_{1}, \ldots, n_{r}\right) \in \mathcal{N}_{r}$ - obtemos um refinamento de $\left\{S_{\xi}^{r-1} \mid \xi \in \sigma\right\}$ - digamos, $\left\{S_{\xi}^{r} \mid \xi \in \sigma\right\}$ - que é um sistema $\left(n_{1}, \ldots, n_{r}, 0\right)$-canônico com respeito a $\mathscr{P}$ para todo $\left(n_{1}, \ldots, n_{j}\right) \in \mathcal{N}_{j}$, com $j \in\{1, \ldots, r\}$. Assim, ao final do processo - tendo aplicado o lema A.6 um total de $1 \cdot m_{1}+\ldots+n \cdot m_{n}$ vezes —, obtemos uma partição $\left\{S_{\xi} \mid \xi \in \sigma\right\}$ que é um sistema $\left(n_{1}, \ldots, n_{r}, 0\right)$ canônico com respeito a $\mathscr{P}$ para quaisquer $r \in\{1, \ldots, n\}$ e $n_{1}, \ldots, n_{r} \in\{1, \ldots, n\}$ com $n_{1}+\ldots+n_{r}=n$.

Isto conclui a demonstração, pois $\left\{S_{\xi} \mid \xi \in \sigma\right\}$ é, então, uma partição de $S=\bigcup\left\{S_{\xi} \mid \xi \in\right.$ $\sigma\} \subseteq T$ que satisfaz as condições requeridas.

q.e.d. 


\section{Apêndice B}

\section{Sobre um exemplo de A. Dow}

Em [5], ao discutir as hipóteses de um teorema de reflexão da função cardinal $\chi$ - nesta dissertação, o teorema 2.3.7 - no caso $\kappa=\omega$, A. Dow afirma existir um exemplo - e esboça sua construção - para justificar a consistência de não se poder substituir "inicialmente$\omega_{1}$-compacto" por "enumeravelmente compacto e de tightness enumerável" nas hipóteses do mesmo. Mais precisamente, Dow sugere uma construção, a partir de $V=L$, de um espaço topológico $(X, \tau)$ tal que:

(i) $(X, \tau)$ é regular; ${ }^{1}$

(ii) $(X, \tau)$ é enumeravelmente compacto;

(iii) $t(X)=\omega ;$

(iv) $\chi(X)>\omega$;

(v) $\chi(Y)=\omega$ para todo $Y \in[X]^{\leq \omega_{1}}$.

\footnotetext{
${ }^{1} \mathrm{O}$ fato de que o espaço é regular não é explicitado na descrição do exemplo; no entanto, como o espaço descrito é $T_{1}$ e o teorema 2.3.7 contém a hipótese de o espaço ser $T_{3}$, presume-se que se queira que o exemplo apresentado também o seja.
} 
A construção do exemplo baseia-se num resultado de [6], para o qual² é necessária a seguinte definição:

Definição B.1. Sejam $\alpha$ e $\beta$ ordinais. Dizemos que $f \in{ }^{\alpha} \omega$ e $g \in{ }^{\beta} \omega$ são paralelas e denotamos tal fato através do símbolo $f \| g-s e$, e somente se, existe $s \in \omega$ tal que $|f(\gamma)-g(\gamma)|<s$ para todo $\gamma \in \alpha \cap \beta$. Dizemos ainda que um conjunto $\mathcal{F} \subseteq \bigcup_{\alpha \in \text { Ord }}{ }^{\alpha} \omega$ é uma família paralela se, e somente se, quaisquer $f, g \in \mathcal{F}$ são paralelas.

Antes de procedermos ao teorema, notemos um fato que será útil mais à frente.

Lema B.2. Sejam $\alpha, \beta$ e $\gamma$ ordinais com $\gamma \notin \alpha \cap \beta$. Se $f \in{ }^{\alpha} \omega, g \in{ }^{\beta} \omega$ e $h \in{ }^{\gamma} \omega$ são tais que $f \| h$ e $g \| h$, então $f \| g$.

Demonstração. Sejam $f, g$ e $h$ como no enunciado, e fixe $r, s \in \omega$ tais que $|f(\xi)-h(\xi)|<r$ para todo $\xi \in \alpha \cap \gamma$ e $|g(\xi)-h(\xi)|<s$ para todo $\xi \in \beta \cap \gamma$.

Como $\gamma \notin \alpha \cap \beta$, então $\alpha \cap \beta \subseteq \gamma$ - pois $\alpha \cap \beta$ é um ordinal. Assim, dado $\xi \in \alpha \cap \beta$ arbitrário, tem-se que $\xi \in \alpha \cap \gamma$ e $\xi \in \beta \cap \gamma$, de modo que $|f(\xi)-h(\xi)|<r$ e $|h(\xi)-g(\xi)|<s$; segue então da desigualdade triangular que $|f(\xi)-g(\xi)| \leq|f(\xi)-h(\xi)|+|h(\xi)-g(\xi)|<r+s$, de modo que $f \| g$, uma vez que $\xi$ é arbitrário.

q.e.d.

O teorema pode então ser enunciado da seguinte maneira:

Teorema B.3. Assuma $V=L$. Então existe uma família paralela $\left\{f_{\alpha} \in{ }^{\alpha} \omega \mid \alpha \in \omega_{2}\right\}$ tal que, para toda função $f: \omega_{2} \rightarrow \omega$, existe $\alpha \in \omega_{2}$ satisfazendo $f_{\alpha} \nVdash f$.

Demonstração. Pela teorema 1.1.1, existe $E \subseteq\left\{\alpha \in \omega_{2} \mid c f(\alpha)=\omega\right\}$ estacionário em $\omega_{2}$ para o qual $\square_{\omega_{1}}(E)$ é verdadeira. Seja então $\left(C_{\alpha}\right)_{\alpha \in \lim \left(\omega_{2}\right)}$ uma $\square_{\omega_{1}}(E)$-seqüência. Podemos supor que $\operatorname{tp}\left(C_{\alpha}\right)=\omega$ para todo $\alpha \in E$ : de fato, se, para cada $\alpha \in E$, trocarmos $C_{\alpha}$ por um conjunto $C_{\alpha}^{\prime}$ cofinal em $\alpha$ de tipo-de-ordem $\omega$, teremos que as condições $(i)$ e $(i i)$ da definição

\footnotetext{
${ }^{2} \mathrm{Na}$ verdade, em [5], este resultado é apresentado com outro enunciado, o qual não é uma conseqüência trivial do resultado demonstrado em [6].
} 
de $\square_{\omega_{1}}(E)$-seqüência serão trivialmente satisfeitas, ao passo que a condição $(i i i)$ será verificada em virtude de que $\lim \left(C_{\alpha}^{\prime}\right)=\emptyset$ para todo $\alpha \in E$ e, para quaisquer $\alpha \in \omega_{2}$ e $\gamma \in \lim \left(C_{\alpha}\right)$, tem-se que $\gamma \notin E-$ e, assim, não há caso em que seja necessário verificar a condição de compatibilidade $C_{\gamma}^{\prime}=\gamma \cap C_{\alpha}$ para a nova seqüência.

Construiremos, por indução transfinita, $\left(f_{\alpha}\right)_{\alpha \in \omega_{2}}$ tal que, para todo $\alpha \in \omega_{2}$,

(a) $\left\{f_{\beta} \mid \beta \in \alpha\right\}$ é uma família paralela;

(b) para $\beta, \gamma \in \alpha$ quaisquer, $\left(\beta \in \lim \left(\omega_{2}\right)\right.$ e $\left.\gamma \in \lim \left(C_{\beta}\right)\right) \Rightarrow f_{\gamma}=f_{\beta}\lceil\gamma$.

Naturalmente, $f_{0}=\emptyset$. Em posse de $f_{\alpha}$ para $\alpha \in \omega_{2}$ arbitrário, defina $f_{\alpha+1}=f_{\alpha} \cup\{(\alpha, 0)\}-$ o que mantém válidas as hipóteses de indução ${ }^{3}$. Finalmente, suponha que $\alpha \in \lim \left(\omega_{2}\right) \backslash\{0\}$ seja tal que já tenhamos construído $\left(f_{\beta}\right)_{\beta \in \alpha}$ nas condições requeridas. Há três casos a considerar:

Caso 1: $\alpha \in E$.

Seja $C_{\alpha}=\left\{\alpha_{n} \mid n \in \omega\right\}$ uma ordenação crescente de $C_{\alpha}-$ i.e., tal que $\alpha_{m}<\alpha_{n}$ para quaisquer $m, n \in \omega$ com $m<n-$; podemos supor, sem comprometimento da demonstração, que $\alpha_{0}=0$. Defina então $f_{\alpha}: \alpha \rightarrow \omega$ tal que, para todo $n \in \omega$ e todo $\gamma \in\left[\alpha_{n}, \alpha_{n+1}\left[\right.\right.$, se tenha que $f(\gamma)=f_{\alpha_{n+1}}(\gamma)+n$. Nestas condições, segue que $\left\{f_{\beta} \mid \beta \leq \alpha\right\}$ é uma família paralela - basta notar que $f_{\alpha} \| f_{\alpha_{n}}$ para todo $n \in \omega$ e aplicar o lema B.2 - ; além disso, a condição (b) da indução é satisfeita devido ao fato de que, comotp $\left(C_{\alpha}\right)=\omega$, então $\lim \left(C_{\alpha}\right)=\emptyset$.

Caso 2: $\alpha \notin E$ e $\lim \left(C_{\alpha}\right)$ é cofinal em $\alpha$.

Pela condição (iii) da definição de $\square_{\omega_{1}}(E)$-seqüência, tem-se que, se $\gamma_{1} \in \lim \left(C_{\alpha}\right)$, então $C_{\gamma_{1}}=\gamma_{1} \cap C_{\alpha}$; logo, se $\gamma_{2} \in \lim \left(C_{\alpha}\right)$ é tal que $\gamma_{2}<\gamma_{1}$, segue que $\gamma_{2} \in \lim \left(C_{\gamma_{1}}\right)$ e, portanto - pela condição $(b)$ da hipótese de indução - $f_{\gamma_{2}}=f_{\gamma_{1}} \uparrow \gamma_{2}$. Assim sendo, temos que $\left\{f_{\gamma} \mid \gamma \in \lim \left(C_{\alpha}\right)\right\}$ é uma família de funções duas a duas compatíveis; defina então $f_{\alpha}=\bigcup_{\gamma \in \lim \left(C_{\alpha}\right)} f_{\gamma}$ - que é uma função de domínio $\alpha$, uma vez que $\lim \left(C_{\alpha}\right)$ é

\footnotetext{
${ }^{3}$ Note que, se $\beta \in \lim \left(\omega_{2}\right)$ e $\gamma \in \lim \left(C_{\beta}\right)$, então $\gamma$ é um ordinal limite — posto que $C_{\beta}$ é c.u.b. em $\beta$.
} 
cofinal em $\alpha$. É fácil ver que, desta forma, as condições da hipótese de indução são satisfeitas por $\left(f_{\beta}\right)_{\beta \leq \alpha}$.

Caso 3: $\alpha \notin E$ e $\lim \left(C_{\alpha}\right)$ não é cofinal em $\alpha$.

Neste caso, sendo $\gamma=\sup \left(\lim \left(C_{\alpha}\right)\right)$, tem-se que $\gamma \in \lim \left(C_{\alpha}\right)$ - pois $C_{\alpha}$ é c.u.b. em $\alpha$-; assim, segue da condição $(i i i)$ da definição de $\square_{\omega_{1}}(E)$-seqüência que existe uma seqüência estritamente crescente $\left(\alpha_{n}\right)_{n \in \omega}$ em $\alpha$ tal que $\alpha_{0}=\gamma, \sup _{n \in \omega} \alpha_{n}=\alpha$ e $C_{\alpha}=$ $C_{\gamma} \cup\left\{\alpha_{n} \mid n \in \omega\right\}$. Definindo então

$$
f_{\alpha}=f_{\gamma} \cup \bigcup_{n \in \omega}\left(f _ { \alpha _ { n + 1 } } \left\lceil\left[\alpha_{n}, \alpha_{n+1}[)\right.\right.\right.
$$

segue novamente da hipótese de indução e do lema B.2 que $\left(f_{\beta}\right)_{\beta \leq \alpha}$ satisfaz as condições (a) e $(b)$.

Tendo então construído a família paralela $\left\{f_{\alpha} \mid \alpha \in \omega_{2}\right\}$, provemos que a mesma satisfaz a condição requerida.

Tome $f: \omega_{2} \rightarrow \omega$ arbitrária. Como $c f\left(\omega_{2}\right)=\omega_{2}>\omega$, existe $n \in \omega$ tal que $A_{n}=f^{-1}[\{n\}]$ é cofinal em $\omega_{2}$. Fixe então um tal $n \in \omega$; afirmamos que existe $\alpha \in E$ tal que $\alpha \cap A_{n}$ é cofinal em $\alpha$.

Suponha, por absurdo, que não exista um tal $\alpha$. Temos então que a função $g: E \rightarrow \omega_{2}$ definida por $g(\alpha)=\sup \left(\alpha \cap A_{n}\right)$ verifica $g(\alpha)<\alpha$ para todo $\alpha \in E \backslash\{0\}$; assim, pelo lema pressing-down, existe $\beta \in \omega_{2}$ tal que $g^{-1}[\{\beta\}]$ é estacionário — e, portanto, cofinal — em $\omega_{2}$. Mas, como $g$ é uma função crescente, isto implica que $A_{n} \subseteq[0, \beta]$ - o que é um absurdo, pois $A_{n}$ é cofinal em $\omega_{2}$.

Tomando então um tal $\alpha \in E$, temos que $f_{\alpha} \nVdash f$, pois $f\left[\alpha \cap A_{n}\right]=\{n\}$ e, por construção, $f_{\alpha}\left[\alpha \cap A_{n}\right]$ é ilimitado em $\omega . \quad$ q.e.d.

Em posse de tal família, podemos então definir o espaço em questão.

Assuma $V=L$ e tome uma família $\left\{f_{\alpha} \in{ }^{\alpha} \omega \mid \alpha \in \omega_{2}\right\}$ como no teorema anterior. Para cada $\alpha \in \omega_{2}$, considere $A_{0}^{\alpha}=\left\{(\beta, m) \in \alpha \times \omega \mid m \leq f_{\alpha}(\beta)\right\}$ e, para todo $n \in \omega \backslash\{0\}$, 
$A_{n}^{\alpha}=\left\{\left(\beta, f_{\alpha}(\beta)+n\right) \mid \beta \in \alpha\right\}$. Dow afirma que, através de um procedimento baseado na construção da reta de Ostaszewski ${ }^{4}$, é possível definir uma topologia $T_{2}$, localmente enumerável e localmente compacta ${ }^{5}$ sobre $\omega_{2} \times \omega$ tal que, para todo $\lambda \in \omega_{2} \operatorname{com} c f(\lambda)=\omega_{1}$, o subespaço $\lambda \times \omega$ é enumeravelmente compacto e, além disso, $A_{n}^{\lambda}$ é aberto e fechado em $\lambda \times \omega$ para todo $n \in \omega$. Em seguida, tomando-se $p \notin \omega_{2} \times \omega$, considera-se a topologia $\tau$ sobre $X=\left(\omega_{2} \times \omega\right) \cup\{p\}$ tal que $\tau^{\omega_{2} \times \omega}$ é a topologia acima descrita, $\omega_{2} \times \omega \in \tau$ e, para todo $U \subseteq X$ com $p \in U$, tem-se que $U$ é uma vizinhança de $p$ em $(X, \tau)$ se, e somente se, $\forall \alpha \in \omega_{2} \exists n \in \omega\left(\bigcup_{m=n}^{\infty} A_{m}^{\alpha}\right) \subseteq U$; note que tal definição para as vizinhanças de $p$ em $(X, \tau)$ é legítima, uma vez que a intersecção de duas tais vizinhanças é também uma vizinhança de $p$ em $(X, \tau)$.

Em primeiro lugar, notemos que é impossível que se tenha uma tal topologia sobre $\omega_{2} \times \omega$. De fato, se, para um certo $\lambda \in \omega_{2}$, os elementos de $\mathscr{C}=\left\{A_{n}^{\lambda} \mid n \in \omega\right\}$ são abertos e fechados, então $\mathscr{C}$ é um recobrimento enumerável de $\lambda \times \omega$ cujos elementos são dois a dois disjuntos, de sorte que $\lambda \times \omega$ não pode ser enumeravelmente compacto.

Uma primeira tentativa de se evitar esse problema seria, ao invés de impor que todo $A_{n}^{\lambda}$ seja aberto e fechado para todo $\lambda \in \omega_{2} \operatorname{com} c f(\lambda)=\omega_{1}$, impor que todo $B_{n}^{\lambda}=\bigcup_{m=n}^{\infty} A_{m}^{\lambda}$ o seja - uma vez que esta é uma condição mais fraca que possibilita a compatibilidade com a definição das vizinhanças de $p \operatorname{em}(X, \tau)$.

Ainda assim, tal alteração não resolve um outro problema: $(X, \tau)$ não verifica $T_{2}$ - e, assim, também não verifica $T_{3}$, uma vez que verifica $T_{1}$.

Para averiguar este fato, tome $\alpha \in \omega_{2}$ arbitrário e sejam $H=\{(\alpha, n) \mid n \in \omega\}$ e $\lambda=\alpha+\omega_{1}$. Como $\lambda \times \omega$ é, por hipótese, enumeravelmente compacto - uma vez que $c f(\lambda)=\omega_{1}$, segue da

\footnotetext{
${ }^{4}$ Vide, e.g., [28], seção 5.3.

${ }^{5}$ Note que, com uma tal topologia, $\chi\left(\omega_{2} \times \omega\right)=\omega$. De fato, se $U=\left\{q_{n} \mid n \in \omega\right\}$ é uma vizinhança enumerável e compacta de um certo $q \in \omega_{2} \times \omega$, então $\left\{\left\{q_{n} \mid n \in \omega\right.\right.$ e $\left.\left.m \leq n\right\} \mid m \in \omega\right\}$ é uma pseudobase para $q$ em $\omega_{2} \times \omega$; como $U$ é compacta, o afirmado decorre da proposição 2.3 .12 e do fato de que, se $U$ é aberto em $\omega_{2} \times \omega$ e $q \in U$, então todo sistema fundamental de vizinhanças para $q$ em $U$ é um sistema fundamental de vizinhanças para $q$ em $\omega_{2} \times \omega$.
} 
proposição 1.2.5 que $H \subseteq \lambda \times \omega$ admite ponto de acumulação ${ }^{6} \mathrm{em} \lambda \times \omega$ - digamos, $q$. Mas, pela maneira como foram definidas as vizinhanças de $p$ em $(X, \tau)$, tem-se que $H \backslash U$ é finito para toda vizinhança $U$ de $p$; assim sendo, $p$ e $q$ não podem admitir vizinhanças disjuntas em $(X, \tau)$.

$\mathrm{Na}$ verdade, este problema é um pouco mais grave do que pode aparentar a princípio: mesmo que seja adotada uma outra definição para as vizinhanças de $p$ em $(X, \tau)$ - que possa eventualmente tornar $(X, \tau)$ um espaço de Hausdorff - , as demais condições requeridas entre as quais incluímos algumas das condições sobre o espaço procurado descritas no começo deste apêndice - implicam que, se $(X, \tau)$ for um espaço $T_{3}$, então $\chi(p, X)=\omega-\mathrm{e}$, portanto, $\chi(X)=\omega$, de modo que $(X, \tau)$ não é um exemplo de espaço nas condições desejadas.

Tal fato decorre do seguinte resultado, devido a O. T. Alas:

Lema B.4. Seja $\tau$ uma topologia sobre $X=\left(\omega_{2} \times \omega\right) \cup\{p\}-\operatorname{com} p \notin \omega_{2} \times \omega-$ tal que:

(i) $(X, \tau)$ é regular;

(ii) $t(X) \leq \omega_{1}$;

(iii) $\chi(Y)=\omega$ para todo $Y \in[X]^{\leq \omega_{1}}$;

(iv) para todo $\lambda \in \omega_{2}$ tal que $c f(\lambda)=\omega_{1}$, o subespaço $\lambda \times \omega$ é enumeravelmente compacto.

Então p é um ponto isolado de $(X, \tau)$.

Demonstração. Em primeiro lugar, afirmamos que, para todo $\lambda \in \omega_{2} \operatorname{com} c f(\lambda)=\omega_{1}$, tem-se que $p$ é um ponto isolado no subespaço $(\lambda \times \omega) \cup\{p\}$.

\footnotetext{
${ }^{6}$ Note que, como $c f\left(\omega_{2}\right)>\omega$ e $\left\{\lambda \in \omega_{2} \mid c f(\lambda)=\omega_{1}\right\}$ é ilimitado em $\omega_{2}$, tem-se na verdade que todo subconjunto infinito e enumerável de $\omega_{2} \times \omega$ admite ponto de acumulação em $\omega_{2} \times \omega$, de modo que $\omega_{2} \times \omega$ é, também pela proposição 1.2.5, enumeravelmente compacto.

Note ainda que, em espaços $T_{1}$, os conceitos de ponto de acumulação e ponto de acumulação completo são equivalentes para subconjuntos de cardinalidade $\omega$.
} 
De fato, fixe um tal $\lambda$ arbitrário. Temos que o subespaço $Y_{\lambda}=(\lambda \times \omega) \cup\{p\}$ verifica $T_{3}$ e, pela hipótese (iii), tem caráter enumerável; assim, $p$ admite um sistema fundamental de vizinhanças abertas $^{7}\left\{V_{n} \mid n \in \omega\right\}$ em $Y_{\lambda}$ tal que $\overline{V_{n+1}} Y_{\lambda} \subseteq V_{n}$ para todo $n \in \omega$. Como $Y_{\lambda}$ verifica $T_{1}$, temos então que $\{p\}=\bigcap_{n \in \omega} V_{n}=\bigcap_{n \in \omega}{\overline{V_{n}}}^{Y_{\lambda}}$; portanto,

$$
\lambda \times \omega=Y_{\lambda} \backslash\{p\}=Y_{\lambda} \backslash\left(\bigcap_{n \in \omega}{\overline{V_{n}}}^{Y_{\lambda}}\right)=\bigcup_{n \in \omega}\left(Y_{\lambda} \backslash{\overline{V_{n}}}^{Y_{\lambda}}\right)
$$

e, como $\lambda \times \omega$ é, pela hipótese $(i v)$, enumeravelmente compacto, segue que existe $m \in \omega$ tal que

$$
\lambda \times \omega=\bigcup_{n=0}^{m}\left(Y_{\lambda} \backslash{\overline{V_{n}}}^{Y_{\lambda}}\right)=Y_{\lambda} \backslash{\overline{V_{m}}}^{Y_{\lambda}} \subseteq Y_{\lambda} \backslash V_{m},
$$

de modo que $p \in V_{m} \subseteq Y_{\lambda} \backslash(\lambda \times \omega)=\{p\}$, i.e., $V_{m}=\{p\}$.

Assim sendo, suponha, por absurdo, que $p$ não seja isolado em $(X, \tau)$. Então $p \in \overline{\omega_{2} \times \omega}$, $\operatorname{logo}$ — pela hipótese $(i i)$ — existe $A \in\left[\omega_{2} \times \omega\right] \leq \omega_{1}$ tal que $p \in \bar{A}$. Como $|A|<\omega_{2}$ e $\omega_{2}$ é um cardinal regular, tem-se que existe um ordinal $\alpha \in \omega_{2}$ tal que $A \subseteq \alpha \times \omega$; tomando-se então $\lambda=\alpha+\omega_{1}$, segue que $c f(\lambda)=\omega_{1}$ e, portanto, $p$ é um ponto isolado em $(\lambda \times \omega) \cup\{p\} \supseteq A \cup\{p\}$. Isto implica que $p \notin \bar{A}$, uma contradição.

q.e.d.

Uma nova modificação na construção que vise obter um espaço nas condições desejadas envolve, portanto, abdicar da condição $(i v)$ do lema anterior - uma vez que todas as outras são essenciais ao exemplo. Como o que se busca é uma topologia que torne $X$ um espaço enumeravelmente compacto - e, em princípio, não se exige tal condição sobre subespaços de $X$-, poderíamos tentar proceder da seguinte maneira:

Consideremos a mesma definição anterior para as vizinhanças de $p$ em $(X, \tau)$. No entanto, ao invés de termos que todo subconjunto infinito e enumerável de $\omega_{2} \times \omega$ admite ponto de acumulação em $\omega_{2} \times \omega$ - o que, como já foi observado, acarreta $(X, \tau) \notin \mathcal{T}_{2}$ 一, podemos

\footnotetext{
${ }^{7}$ Para tanto, fixe sistemas fundamentais de vizinhanças $\mathcal{U}$ e $\mathcal{F}$ para $p$ em $Y_{\lambda}$ tais que $\mathcal{U}$ é enumerável digamos, $\mathcal{U}=\left\{U_{n} \mid n \in \omega\right\}$ - e todo elemento de $\mathcal{F}$ é fechado em $Y_{\lambda}$. Basta então definir $V_{0}=U_{0}$ e, para cada $n \in \omega$, tomar $W_{n} \in \mathcal{F}$ e $V_{n+1} \in \tau_{p}^{Y_{\lambda}}$ tais que $V_{n+1} \subseteq W_{n} \subseteq U_{n} \cap V_{n}$.
} 
tentar impor, na construção da topologia de $\omega_{2} \times \omega$, que haja ponto de acumulação em $\omega_{2} \times \omega$ apenas para os subconjuntos infinitos e enumeráveis de $\omega_{2} \times \omega$ dos quais $p$ não será ponto de acumulação - é possível determinar quais são tais subconjuntos, uma vez que as vizinhanças de $p$ já foram definidas a priori.

Porém, neste caso, ainda que se tenha então $(X, \tau) \in \mathcal{T}_{2}$, o seguinte problema persiste: supondo que se obtenha uma tal topologia ${ }^{8}$ sobre $X$ - digamos, $\tau-$, as condições " $(X, \tau)$ é $T_{3}$ " e " $\chi(p, X)>\omega$ ", necessárias ao exemplo procurado, não podem ocorrer simultaneamente. Para tanto, basta notar que, se definirmos $G_{m}=\left\{(\alpha, n) \in \omega_{2} \times \omega \mid m \leq n\right\}$ para todo $m \in \omega$, então $\left\{G_{m} \mid m \in \omega\right\}$ é uma pseudobase para $p$ em $(X, \tau)$, de modo que $\psi(p, X)=\omega$ e, pela proposição 2.3.12, como $(X, \tau)$ é enumeravelmente compacto, então $(X, \tau) \in \mathcal{T}_{3}$ implica $\chi(p, X)=\omega$.

\section{B.1 Propriedades do espaço $(X, \tau)$}

Após esta série de considerações acerca deste exemplo, demonstraremos agora que, adotando a definição proposta para as vizinhanças de $p$ em $(X, \tau)$, são satisfeitas as condições $(i i i),(i v)$ e $(v)$ descritas no início deste apêndice, contanto que a topologia considerada no subespaço $\omega_{2} \times \omega$ seja compatível com as vizinhanças de $p$ e verifique $\chi(Y)=\omega$ para todo $Y \in\left[\omega_{2} \times \omega\right]^{\leq \omega_{1}}$ e $t(q, X)=\omega$ para todo $q \in \omega_{2} \times \omega$. Note que, em virtude da última observação feita antes do início desta seção, as condições $(i)$ e $(i i)$ são incompatíveis, uma vez que a condição $(i v)$ é verificada.

Cabe ressaltar que, ainda que tenhamos concluído que este exemplo não pode verificar todas as propriedades desejadas, esta seção foi incluída por apresentar técnicas interessantes para a abordagem do problema em questão.

\footnotetext{
${ }^{8}$ Infelizmente, as tentativas neste sentido realizadas no desenvolvimento do presente trabalho não obtiveram êxito.
} 
Antes de mais nada, vamos introduzir algumas notações e terminologias que serão utilizadas nesta seção, assim como enunciar algumas afirmações que nos auxiliarão a tratar este exemplo.

Seja $\mathcal{V}_{p}$ o conjunto de todas as vizinhanças de $p$ em $(X, \tau)$. Dados $\alpha \in \omega_{2}$ e $n \in \omega$ arbitrários, seja $B_{n}^{\alpha}=\left\{(\beta, m) \in \omega_{2} \times \omega \mid m \geq f_{\alpha}(\beta)+n\right\}$.

Lema B.1.1. Para cada $V \in \mathcal{V}_{p}$, existe $g: \omega_{2} \rightarrow \omega$ tal que $\left\{(\alpha, n) \in \omega_{2} \times \omega \mid n \geq g(\alpha)\right\} \subseteq V$.

Demonstração. Basta notar que, se $V \in \mathcal{V}_{p}$, então $\{n \in \omega \mid(\alpha, n) \notin V\}$ é finito para cada $\alpha \in \omega_{2}$.

q.e.d.

Defina então, para cada vizinhança $V \in \mathcal{V}_{p}$,

$$
\begin{aligned}
g_{V}: \omega_{2} & \rightarrow \omega \\
\alpha & \mapsto \sup \{n \in \omega \mid(\alpha, n) \notin V\}+1 .
\end{aligned}
$$

Dada uma função $f: \omega_{2} \rightarrow \omega$, seja $V_{f}:=\{p\} \cup\left\{(\alpha, n) \in \omega_{2} \times \omega \mid n>f(\alpha)\right\}$. Diremos que $f$ define uma vizinhança de $p$ em $(X, \tau)$ se, e somente se, $V_{f} \in \mathcal{V}_{p}$. Note que, com esta terminologia, o seguinte resultado decorre imediatamente da definição das vizinhanças de $p$ em $(X, \tau)$ :

Lema B.1.2. (a) Seja $f: \omega_{2} \rightarrow \omega$. Temos que $f$ define uma vizinhança de $p$ em $(X, \tau)$ se, e somente se,

$$
\forall \alpha \in \omega_{2} \exists n \in \omega \forall \beta \in \alpha\left(f(\beta) \leq f_{\alpha}(\beta)+n\right) .
$$

(b) Para toda $V \in \mathcal{V}_{p}$, tem-se que $g_{V}$ define uma vizinhança de $p$ em $(X, \tau)$.

Seja ainda, para todo $\alpha \in \omega_{2}$,

$$
\begin{aligned}
\tilde{f}_{\alpha}: \omega_{2} & \rightarrow \omega \\
\beta & \mapsto \begin{cases}f_{\alpha}(\beta), & \text { se } \beta \in \alpha ; \\
0, & \text { se } \beta \in \omega_{2} \backslash \alpha\end{cases}
\end{aligned}
$$


Note que, como conseqüência do item $(a)$ no lema anterior, tem-se que, para todo $\alpha \in \omega_{2}$, a função $\tilde{f}_{\alpha}$ define uma vizinhança de $p$ em $(X, \tau)$ - uma vez que a família $\left\{f_{\alpha} \mid \alpha \in \omega_{2}\right\}$ é paralela.

Procedamos então à demonstração das condições $(i i i),(i v)$ e $(v)$.

Comecemos pela condição $(v)$.

Tome $Y \in[X]^{\leq \omega_{1}}$ arbitrário. Como $\omega_{2}$ é um cardinal regular, existe então $\gamma \in \omega_{2}$ tal que $Y \subseteq(\gamma \times \omega) \cup\{p\}$; como $\chi$ é uma função cardinal monótona, podemos supor, sem perda de generalidade, que $Y=(\gamma \times \omega) \cup\{p\}$.

Por hipótese, $\chi(\gamma \times \omega)=\omega$; assim, para concluir que $\chi(Y)=\omega$, basta verificar que $\chi(p, Y)=\omega$ - uma vez que, como $\omega_{2} \times \omega$ é aberto em $(X, \tau)$, então $\chi(q, Y)=\chi(q, \gamma \times \omega)=\omega$ para todo $q \in \gamma \times \omega$. Mas, pelo paralelismo da família $\left\{f_{\alpha} \mid \alpha \in \omega_{2}\right\}$, tem-se que $B_{n}^{\gamma}$ é uma vizinhança de $p$ em $Y$ para todo $n \in \omega$, e segue então diretamente da definição de $\mathcal{V}_{p}$ que $\left\{B_{n}^{\gamma} \mid n \in \omega\right\}$ é um sistema fundamental de vizinhanças para $p$ em $Y$. Portanto, $\chi(p, Y)=\omega$.

Passemos agora à condição $(i i i)$.

Como, por hipótese, $t(q, X)=\omega$ para todo $q \in \omega_{2} \times \omega$, basta provar que $t(p, X)=\omega$. Para tanto, tome $A \subseteq X$ arbitrário tal que $p \in \bar{A}$; devemos mostrar que existe um subconjunto enumerável de $A$ ao qual $p$ seja aderente.

Se $p \in A$, a tese é trivialmente verdadeira. Se existir $\beta \in \omega_{2}$ tal que $A \cap\{(\beta, n) \mid n \in \omega\}$ é infinito, a conclusão também é imediata, pois, nesse caso, $p$ é aderente a $A \cap\{(\beta, n) \mid n \in \omega\}$ - e.g, pelo lema B.1.1.

Suponha agora que nenhuma das condições anteriores ocorre, e considere então

$$
\begin{aligned}
f: \omega_{2} & \rightarrow \omega \\
\beta & \mapsto \sup \{n \in \omega \mid(\beta, n) \in A\}
\end{aligned}
$$


Por hipótese, o conjunto $P=\left\{\alpha \in \omega_{2} \mid f_{\alpha} \nVdash f\right\}$ é não-vazio. Suponha, por absurdo, que, para todo $\alpha \in P$, exista $m \in \omega$ tal que $f(\delta) \leq f_{\alpha}(\delta)+m$ para todo $\delta \in \alpha$. Como $f_{\beta} \| f$ para todo $\beta \in \omega_{2} \backslash P$, temos - pela definição de $\|$ - que, para todo tal $\beta$, existe $n \in \omega$ tal que $f(\delta) \leq f_{\beta}(\delta)+n$ para todo $\delta \in \beta$. Mas então o lema B.1.2(a) implica que $f$ define uma vizinhança de $p$ em $(X, \tau)$, de modo que $V_{f} \in \mathcal{V}_{p}$ é tal que $V_{f} \cap A=\emptyset$, o que contradiz a hipótese de que $p \in \bar{A}$.

Portanto, existe $\alpha \in P$ tal que, para todo $m \in \omega$, existe $\delta_{m} \in \alpha$ tal que $f\left(\delta_{m}\right)>f_{\alpha}\left(\delta_{m}\right)+m$. Fixe então um tal $\delta_{m}$ para cada $m \in \omega$ e considere $D=\left\{\left(\delta_{m}, f\left(\delta_{m}\right)\right) \mid m \in \omega\right\} \subseteq A$.

Afirmamos que $p \in \bar{D}$. De fato, se, por absurdo, existisse $V \in \mathcal{V}_{p}$ tal que $V \cap D=\emptyset$, teríamos que $g_{V}\left(\delta_{m}\right)>f\left(\delta_{m}\right)>f_{\alpha}\left(\delta_{m}\right)+m$ para todo $m \in \omega$, o que, pelo lema B.1.2, contradiz a hipótese de que $V \in \mathcal{V}_{p}$.

Assim, $t(p, X)=\omega$, como afirmado.

Finalmente, tratemos da condição $(i v)$.

Provaremos que $\chi(p, X) \geq \omega_{2}$. Tome $\mathcal{U} \in\left[\mathcal{V}_{p}\right]^{\leq \omega_{1}}$ arbitrário; vamos determinar uma vizinhança de $p$ em $(X, \tau)$ que não contém nenhum elemento de $\mathcal{U}$.

Para cada $U \in \mathcal{U}$, fixe $\alpha_{U} \in \omega_{2}$ tal que $f_{\alpha_{U}} \nVdash g_{U}-$ um tal $\alpha_{U}$ existe por hipótese. Considere então $\alpha=\sup \left\{\alpha_{U} \mid U \in \mathcal{U}\right\}$ - temos que $\alpha \in \omega_{2}$, pois $|\mathcal{U}| \leq \omega_{1}$ e $\omega_{2}$ é regular. Afirmamos que, para todo $U \in \mathcal{U}$, tem-se que $U \nsubseteq V_{\tilde{f}_{\alpha}}$ - o que conclui a demonstração, uma vez que, em virtude das observações já feitas nesta seção, $V_{\tilde{f}_{\alpha}} \in \mathcal{V}_{p}$.

Suponha, por absurdo, que exista $W \in \mathcal{U}$ tal que $W \subseteq V_{\tilde{f}_{\alpha}}-$ ou, o que é equivalente, $g_{W}(\beta)>\tilde{f}_{\alpha}(\beta)$ para todo $\beta \in \omega_{2}$. Então, em particular, para todo $\beta \in \alpha_{W} \subseteq \alpha$ tem-se que $g_{W}(\beta)>\tilde{f}_{\alpha}(\beta)=f_{\alpha}(\beta)$. Como, por hipótese, $f_{\alpha_{W}} \| f_{\alpha}$, então existe $m \in \omega$ tal que $f_{\alpha}(\beta)+m>f_{\alpha_{W}}(\beta)$ para todo $\beta \in \alpha_{W}$, o que implica que $g_{W}(\beta)+m>f_{\alpha_{W}}(\beta)$ para todo $\beta \in \alpha_{W}$. Mas, como $W \in \mathcal{V}_{p}$, segue do lema B.1.2 que existe $k \in \omega$ tal que $g_{W}(\beta) \leq f_{\alpha_{W}}(\beta)+k$ para todo $\beta \in \alpha_{W}$. Temos então que, para todo $\beta \in \alpha_{W}$, ocorre $\left|f_{\alpha_{W}}(\beta)-g_{W}(\beta)\right| \leq \sup \{m, k\}$, de modo que $f_{\alpha_{W}} \| g_{W}$, contrariando a escolha de $\alpha_{W}$. 
Portanto, $\chi(p, X) \geq \omega_{2}$. 


\section{Índice de símbolos}

$\begin{array}{ll}{[X]^{\kappa}, 6} & \lim , 7 \\ {[X]^{<\kappa}, 6} & \text { Ord, } 6 \\ {[X]^{\leq \kappa}, 6} & \psi, 37 \\ \models, 10 & s, 35 \\ \bar{A}, 23 & \mathcal{T}, 6 \\ \bar{A}^{X}, 23 & t, 37 \\ \prec, 10 & \tau_{x}, 22 \\ \square, 8 & \tau^{T}, 22 \\ \uparrow, 6 & \mathcal{T}_{i}, 24 \\ \mathcal{C}_{2}, 30 & T_{i}, 23 \\ c, 36 & t p, 7 \\ C a r d, 6 & w, 35 \\ c f, 8 & \chi, 37 \\ d, 35 & \chi(A, X), 37 \\ e, 36 & \chi(x, X), 37 \\ f[A], 6 & \\ f^{-1}[B], 7 & \\ H_{\theta}, 13 & \\ h \varphi, 34 & \\ \text { int, } 23 & \\ I U, 40 & \\ \kappa^{+}, 7 & \\ L, 35 & \\ & \end{array}$




\section{Índice remissivo}

aderência, 23

aderente, 23

base, 22

c.u.b., 8

cadeia, 12

elementar, 16

contínua, 16

$\epsilon-, 16$

caráter, 37

cardinal

fortemente inacessível, 8

limite, 7

limite forte, 7

regular, 8

singular, 8

sucessor, 7

celularidade, 36

cofinal, 8

cofinalidade, 8

coletivamente de Hausdorff, 25

compacto, 30

completamente regular, 24

densidade, 35

enumerável, 6

enumeravelmente compacto, 28
E-set, 80

espaço de Hausdorff, 24

estacionário, 8

extent, 36

família celular, 25

fechado, 8

fecho, 23

função cardinal, 34

global, 35

local, 36

função-classe, 6

grau de Lindelöf, 35

hereditariamente normal, 25

ilimitado, 8

induz, 22

inicialmente- $\kappa$-compacto, 28

isolado, 22

$\kappa$-coletivamente de Hausdorff, 25

$\kappa$-covering, 19

$\kappa$-fechado, 17

localmente finito, 30

monótona, 34

normal, 24 
ordinal

limite, 7

sucessor, 7

paracompacto, 30

partição, 95

peso, 35

ponto

de acumulação, 22

completo, 23

interior, 23

pontualmente enumerável, 31

propriedade de Darboux, 40

pseudobase, 37

pseudocaráter, 37

recobrimento, 28

aberto, 28

refinamento, 28

aberto, 28

reflete, 40

fortemente, 40

regular

cardinal,- 8

espaço - 24

separável, 35

separado

à direita, 23

à esquerda, 23

seqüência livre, 60 $\sigma$-localmente finita, 31

sistema fundamental de vizinhanças, 22

de um subconjunto, 37

spread, 35

sub-recobrimento, 28

submodelo elementar, 10

tightness, 37

tipo-de-ordem, 7

topologia da ordem, 26

vizinhança, 22

de um subconjunto, 37 


\section{Referências bibliográficas}

[1] Z. Balogh, Locally nice spaces and axiom R, Topology and its Applications 125 (2002), $335-341$.

[2] Z. Balogh, Reflecting point-countable families, Proceedings of the American Mathematical Society 131 (2003), 1289-1296.

[3] H. H. Corson e E. Michael, Metrizability of certain countable unions, Illinois Journal of Mathematics 8 (1964), 351-360.

[4] A. Dow, An empty class of nonmetric spaces, Proceedings of the American Mathematical Society 104 (1988), 999-1001.

[5] A. Dow, An introduction to applications of elementary submodels to topology, Topology Proceedings 13 (1988), 17-72.

[6] A. Dow, I. Juhász e W. Weiss, Integer-valued functions and increasing unions of first countable spaces, Israel Journal of Mathematics 67 (1989), 181-192.

[7] E. K. van Douwen, The integers and topology, Handbook of set-theoretic topology, NorthHolland, Amsterdam, 1984, 111-167.

[8] R. Engelking, General topology, Heldermann, Berlin, 1989.

[9] W. G. Fleissner, On $\lambda$ collection Hausdorff spaces, Topology Proceedings 2 (1977), 445456. 
[10] W. G. Fleissner, Separation properties in Moore spaces, Fundamenta Mathematicae 98 (1978), 279-286.

[11] W. G. Fleissner, Left separated spaces with point-countable bases, Transactions of the American Mathematical Society 294 (1986), 665-677.

[12] A. Fedeli e S. Watson, Elementary submodels and cardinal functions, Topology Proceedings 20 (1995), 91-110.

[13] A. Hajnal e I. Juhász, Some remarks on a property of topological cardinal functions, Acta Mathematica Academiae Scientiarum Hungaricae 20 (1969), 25-37.

[14] A. Hajnal e I. Juhász, Discrete subspaces of topological spaces, II, Indagationes Mathematicae 31 (1969), 18-30.

[15] A. Hajnal e I. Juhász, On spaces in which every small subspace is metrizable, Bulletin de l'Académie Polonaise des Sciences 24 (1976), 727-732.

[16] A. Hajnal e I. Juhász, Having a small weight is determined by the small subspaces, Proceedings of the American Mathematical Society 79 (1980), 657-658.

[17] R. Hodel, Cardinal functions I, Handbook of set-theoretic topology, North-Holland, Amsterdam, 1984, 1-61.

[18] R. E. Hodel e J. E. Vaughan, Reflection theorems for cardinal functions, Topology and its Applications 100 (2000), 47-66.

[19] K. Hrbacek e T. Jech, Introduction to set theory, 3. edição, Monographs and textbooks in pure and applied mathematics no. 220, Marcel Dekker, New York, 1999.

[20] T. Jech, Set theory, 3. edição, Springer, Berlin, 2002.

[21] R. B. Jensen, The fine structure of the constructible hierarchy, Annals of Mathematical Logic 4 (1972), 229-308. 
[22] I. Juhász, Cardinal functions in topology, Mathematical Centre Tracts no 34, Amsterdam, 1971.

[23] I. Juhász, Cardinal functions in topology - ten years later, Mathematical Centre Tracts n. 123 , Amsterdam, 1980.

[24] I. Juhász, Cardinal functions and reflection, Topology Atlas Preprint no 445, 2000.

[25] K. Kunen, Set theory, North-Holland, Amsterdam, 1980.

[26] M. D. Passos, Extensões de submodelos elementares por forcing, Tese de doutorado, Universidade de São Paulo, 2007.

[27] T. Przymusiński, A note on collectionwise normality and product spaces, Colloquium Mathematicum 33 (1975), 65-70.

[28] J. Roitman, Basic S and L, Handbook of set-theoretic topology, North-Holland, Amsterdam, 1984, 295-326.

[29] S. Shelah, Remarks on $\aleph_{1}-C W H$ not CWH first countable spaces, Contemporary Mathematics, vol. 192, American Mathematical Society, Providence, RI, 1996, 103-145.

[30] F. D. Tall, Reflection of topological properties to $\aleph_{1}$, Open problems in topology II, Elsevier, Amsterdam, 2007, 241-247.

[31] V. V. Tkachuk, Spaces that are projective with respect to classes of mappings, Transactions of the Moscow Mathematical Society 50 (1988), 139-156.

[32] J. E. Vaughan, On Dow's reflection theorem for metrizable spaces, Topology Proceedings 22 (1997), 351-361. 